Florida International University

FIU Digital Commons

FIU Electronic Theses and Dissertations

University Graduate School

3-9-2017

\title{
The Effect of Employing Cultural Criticism in the Teaching of British Literature for Chinese Undergraduate English Majors
}

Yu Zhang

Dept. of Teaching and Learning, FIU, yzhan050@fiu.edu

DOI: 10.25148 /etd.FIDC001743

Follow this and additional works at: https://digitalcommons.fiu.edu/etd

Part of the Curriculum and Instruction Commons, Educational Methods Commons, Higher Education Commons, Language and Literacy Education Commons, and the Teacher Education and Professional Development Commons

\section{Recommended Citation}

Zhang, Yu, "The Effect of Employing Cultural Criticism in the Teaching of British Literature for Chinese Undergraduate English Majors" (2017). FIU Electronic Theses and Dissertations. 3198.

https://digitalcommons.fiu.edu/etd/3198 


\section{FLORIDA INTERNATIONAL UNIVERSITY}

Miami, Florida

\section{THE EFFECT OF EMPLOYING CULTURAL CRITICISM IN THE TEACHING OF BRITISH LITERATURE FOR CHINESE UNDERGRADUATE ENGLISH MAJORS}

A dissertation submitted in partial fulfillment of

the requirements for the degree of

DOCTOR OF PHILOSOPHY

in

CURRICULUM AND INSTRUCTION

by

Yu Zhang

2017 
To: Dean Michael R. Heithaus

College of Arts, Sciences and Education

This dissertation, written by Yu Zhang, and entitled The Effect of Employing Cultural

Criticism in the Teaching of British Literature for Chinese Undergraduate English Majors, having been approved in respect to style and intellectual content, is referred to you for judgment.

We have read this dissertation and recommend that it be approved.

Heather Russell

Sarah Mathews

Haiying Long

Linda Spears-Bunton, Major Professor

Date of Defense: March 9, 2017

The dissertation of Yu Zhang is approved.

Dean Michael R. Heithaus
College of Arts, Sciences and Education

College of Arts, Sciences and Education

Andrés G. Gil

Vice President for Research and Economic Development and Dean of the University Graduate School

Florida International University, 2017 
C Copyright 2017 by Yu Zhang

All rights reserved. 


\section{DEDICATION}

This dissertation is dedicated to my dear parents, Ziqiang Zhang (张子强) and Lixia Ge (戈丽霞). They are living thousands of miles from me, but they have always been with me during my years abroad. They have always provided me with unconditional love, care and support.

This dissertation is also dedicated to my dear husband, Dr. Xin Ye (叶歆). His patience, encouragement and support has always accompanied with me on this journey.

This dissertation is also dedicated to my unborn child, Gordon Ye (叶牧辰). I can feel him moving around and kicking while I was writing up the dissertation. I am counting the days till my baby arrives in three months. I believe he will be an intelligent and diligent person like his parents.

Thank you for all my beloved ones. I (张羽) will love you as always. 


\section{ACKNOWLEDGMENTS}

I would like to first extend my deepest appreciation to my committee members, Dr. Linda Spears-Bunton, Dr. Haiying Long, Dr. Sarah Mathews, and Dr. Heather Russell. Dr. Linda Spears-Bunton has provided me mentoring since I was enrolled in this program. She is a great mentor who is always inspiring, supportive and helpful throughout my doctoral study. She also cares about me in my life. She has gone far beyond being my major professor. Dr. Long always provided me her expertise and insight on statistical methods and analysis. Her valuable suggestions and effective feedback on the methodological part made me have a deep understanding of various statistical models. Dr. Mathews and Dr. Russell, those two brilliant and nice ladies generously shared their knowledge in teaching English literature and building a conceptual framework for my study. I am very grateful to have them join in my Committee. They have made this research incorporate deeper perspectives than I would have been able to accomplish alone.

I am also greatly indebted to Dr. Linda Bliss. She is a knowledgeable and insightful scholar who always arouses my interest in research. Every time I submitted a draft to her, she provided feedback that was pertinent, rigorous, critical and constructive. I enjoyed discussing any question I have had in the research with her.

My special thank you for professors at Anhui Agricultural University, Yuming Wang, Huali Xu, Peipei Li. Their helpfulness made the implementation of curriculum design, the data collection process and grading work much smoother. I also want to thank English teacher Wen Shu, a trusted-friend of mine for ten years, for assisting me in grading dozens of students' essays. 
Asides from my Committee and colleagues, I would like to express my sincere gratitude to Dr. Thomas Reio, for his valuable ideas and suggestions in my research, and for his consistent support in my Fellowship applications. I also want to thank you Suzi Barcenas, Caprila Almeida at Office of Graduate Studies. Thank you for your assistance during my dissertation journey.

I would like to thank University of Graduate School at FIU for awarding me Doctoral Evidence Acquisition Fellowship for the Fall 2015 academic year and Doctoral Dissertation Year Fellowship for Summer \& Fall 2016. These fellowships supported me in completion of this dissertation.

My husband, Dr. Xin Ye deserves my thousands of thanks throughout my study. He provided me unconditional support and love when I devoted my time to the research. I enjoyed communicating with him on any idea, thought and perspective for my dissertation. He always has a rational, logical and sober mind. He was willing to listen to me, reading my work, sharing his wonderful ideas with me, and providing his comments to help me improve the overall quality for this work. 


\begin{abstract}
OF THE DISSERTATION
THE EFFECT OF EMPLOYING CULTURAL CRITICISM IN THE TEACHING OF BRITISH LITERATURE FOR CHINESE UNDERGRADUATE ENGLISH MAJORS
\end{abstract} by

Yu Zhang

Florida International University, 2017

Miami, Florida

Professor Linda Spears-Bunton, Major Professor

The traditional literature teaching methods for Chinese English majors are formalism and biographical criticism. These criticisms use an objective approach focused on details about the author, historical context and literary mechanics to analyze literature. These methods neglect the fact that literature comprehension involves readers' active participation. Cultural criticism, as a critical approach, considers influences that readers bring to their engagement with a given literary text. This approach is supposed to fit the classroom settings for cross-cultural literature teaching and learning.

This study was conducted to examine the effect of utilizing cultural criticism to teach British literature among Chinese undergraduate English majors. The effect of employing cultural criticism was reflected in two aspects: students' cultural understandings of literary texts and their literature comprehension. In this study, students' awareness of cultural influences in literary texts from cultural perspectives was evaluated as their cultural understandings; literature comprehension was assessed from students' understanding in context, themes, and textual meaning of literary texts. 
In this study a pedagogy of cultural criticism was developed and implemented in the teaching of a British literature course. Students received instruction through cultural criticism lens for two hours per week over a period of 14 weeks. The instruments included two essay tests concerning the cultural analysis of literary works, and three literature comprehension tests. A quasi-experimental design and a repeated measure mixed-design were used to compare the performance for students in two experimental groups (cultural criticism approach) and one control group (formalist and biographical approach). Various statistical models were applied to data analysis.

The experimental results showed that the cultural criticism approach resulted in better cultural understandings of literary texts and better literature comprehension than the traditional formalist and biographical approach. Another finding is the different performance in cultural understandings of literary texts between the two experimental groups, as the instructor had different proficiency levels in using the cultural criticism approach.

This study has provided evidence that cultural criticism could be a valuable approach to help Chinese undergraduate English majors bridge cultural gaps in their understandings of literature and facilitate literature comprehension. 


\section{TABLE OF CONTENTS}

CHAPTER

PAGE

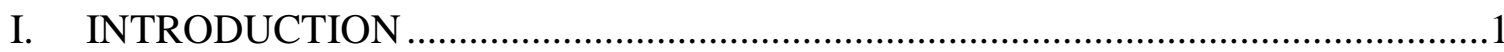

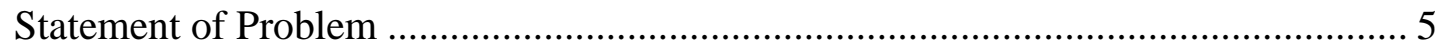

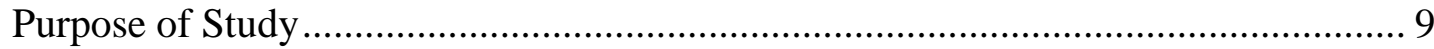

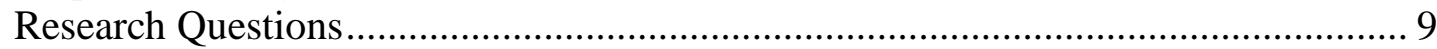

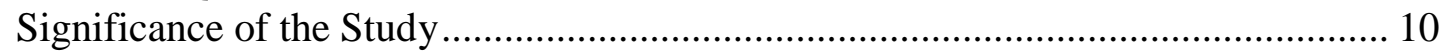

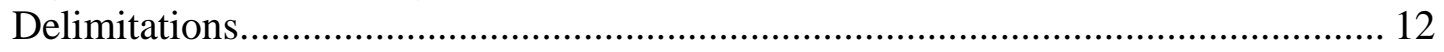

Operational Definitions ..................................................................................... 12

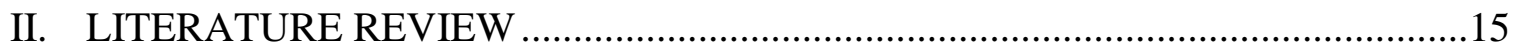

Theoretical Framework............................................................................... 15

Applications of Related Theories in the Classroom Settings .................................... 26

Views of Curriculum ……………………………………………………..... 30

English Literature Curriculum in China …………………….................................... 33

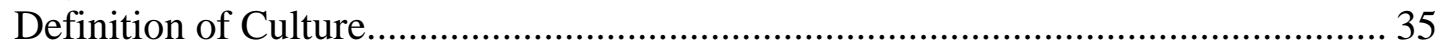

Relationship among Literacy, Literature and Culture …………………………....... 36

Pedagogy Issues and Suggestions in Teaching Literary Texts ................................... 38

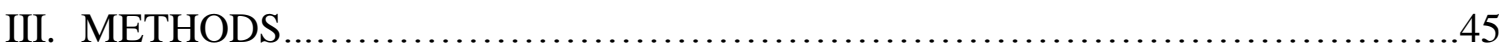

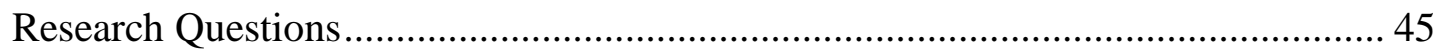

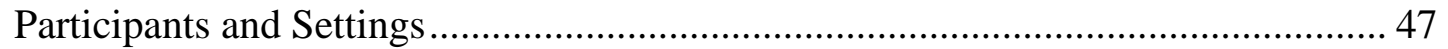

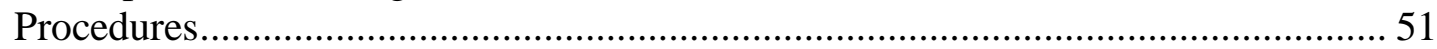

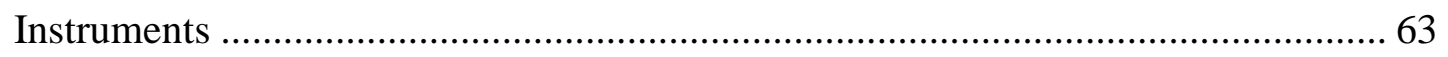

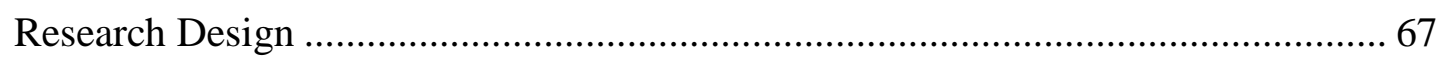

Data Collection and Measurement........................................................................ 68

Research Hypotheses and Statistical Models ........................................................... 77

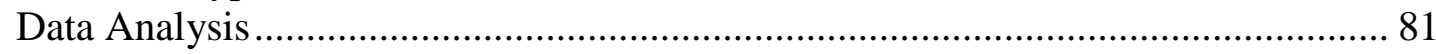

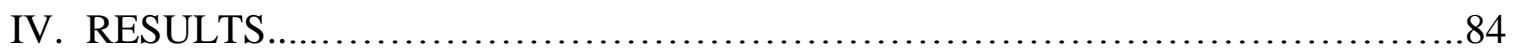

Descriptive Analysis of All Collected Test Scores...................................................... 84

Culture Analysis Tests ..................................................................................... 86

Literature Comprehension Tests......................................................................... 110

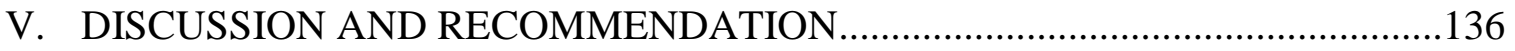

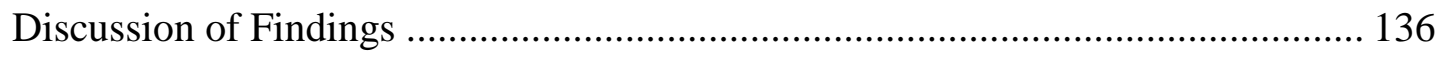

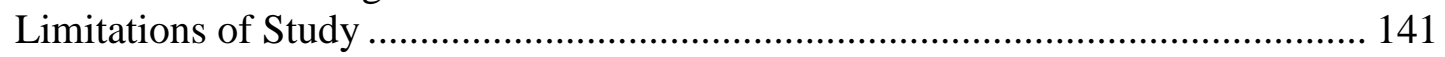

Recommendation for Future Research and Teaching Practice................................ 143

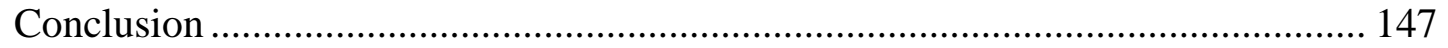

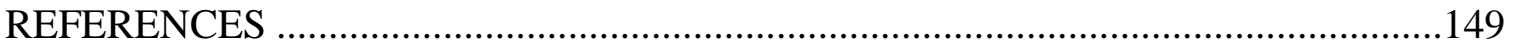

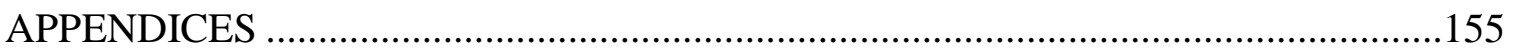

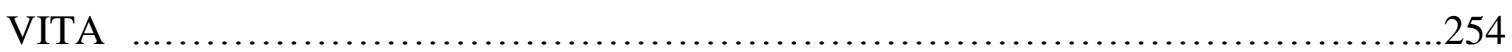




\section{LIST OF TABLES}

TABLE

PAGE

1. Three-group Partition of All Participants in This Study ......................................... 49

2. Reading Schedule for All British Literature Classes ........................................... 52

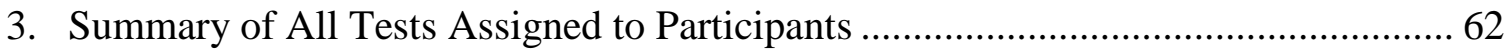

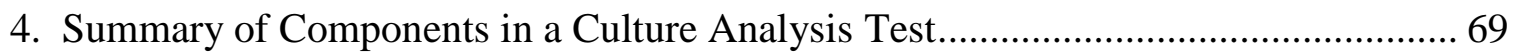

5. Summary of Components in a Literature Comprehension Test................................. 71

6. Selected Results of the Test of Homogeneity of Slopes ........................................ 88

7. Pairwise Differences in Mean Changes for CulTestB Conditioned at Low

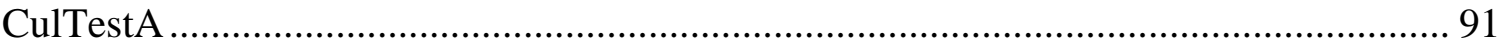

8. Pairwise Differences in Mean Changes for CulTestB Conditioned at Medium

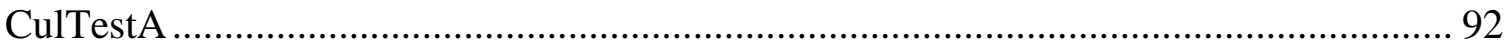

9. Pairwise Differences in Mean Changes for CulTestB Conditioned at High

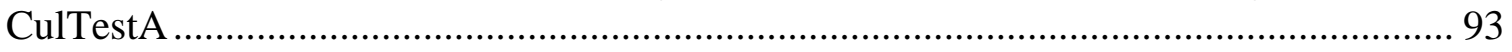

10. Summary of Results of a One-way Analysis of Variance for CulTestB .................. 95

11. The Results of Post hoc Comparisons in Mean Changes for CulTestB .................. 95

12. Summary of Results of a One-way Multivariate Analysis of Covariance for Five

Cultural Components in CulTestB ............................................................................ 99

13. Pairwise Differences in Mean Changes for Five Cultural Components in

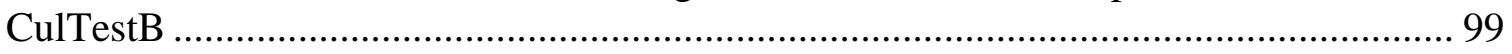

14. Summary of Results of a One-way Analysis of Variance for General

Improvement Scores in CulTests.

15. Pairwise Differences in Mean Changes for General Improvement Scores in

CulTests

16. Summary of Results of a One-way Multivariate Analysis of Variance for

General Improvement Scores in Five Cultural Components of CulTests.

17. The Results of Post Hoc Comparisons in Mean Changes for Improvement Scores

in Three Cultural Components of CulTests 108 
18. Summary of Results of Repeated Measures Analysis of Covariance Mixed

Design for CompTests

19. The Results of the Group Main Effect Pairwise Comparison for CompTests

20. The Results of Pairwise Comparisons for Group*Time Interaction in

CompTests

21. The Results of Pairwise Comparisons for Time*Group Interaction in

CompTests

22. Summary of Results of Repeated Measures Multivariate Analysis Mixed Design of Covariance for CompTests

23. The Results of the Group Main Effect Pairwise Comparison for Three

Subcategories in CompTests

24. The Results of Pairwise Comparisons for Group *Time Interaction in Three

Subcategories of CompTests.

25. The Results of Pairwise Comparisons for Time*Group Interaction in Three

Subcategories of CompTests. 130

26. Descriptive Statistics for CulTestA

27. Descriptive Statistics for CulTestB.

28. Descriptive Statistics for CulTest Improvement scores

29. Descriptive Statistics for Three CompTests 242

30. Descriptive Statistics for TEM4. 242

31. Estimated $95 \%$ Confidence Intervals of Mean in CulTestB Conditioned at Low CulTestA 243

32. Estimated 95\% Confidence Intervals of Mean in CulTestB Conditioned at Medium CulTestA

33. Estimated 95\% Confidence Intervals of Mean in CulTestB Conditioned at High CulTestA

34. Descriptive Statistics for Five Cultural Components in CulTestB

35. Estimated 95\% Confidence Intervals of Mean in Five Cultural Components for CulTestB

36. $95 \%$ Confidence Intervals of Mean for General Improvement Scores in CulTests 245 
37. Descriptive Statistics for Improvement Scores in Five Cultural Components of CulTestB

38. Estimated 95\% Confidence Intervals of Mean for Improvement Scores in Five Cultural Components of CulTests.

39. Estimated 95\% Confidence Intervals of Mean for Group in Three CompTests...... 247

40. Estimated 95\% Confidence Intervals of Mean for Each Group in Different CompTest.

41. Estimated 95\% Confidence Intervals of Mean for Each CompTest in Different Group

42. Descriptive Statistics for Three Subcategories in CompTests

43. Estimated 95\% Confidence Intervals of Mean for Group in Three Subcategories of CompTests

44. Estimated 95\% Confidence Intervals of Mean for Each Group in Three Subcategories of Different CompTest 251

45. Estimated 95\% Confidence Intervals of Mean for Three Subcategories of Each CompTest in Different Group 252 


\section{CHAPTER I}

\section{INTRODUCTION}

Literary texts are usually inhabited cultural contexts (Jodan \& Purves, 1993). Cultural context is the cultural settings in the text, where characters' behaviors may be influenced by the social culture they are living in. Literary works created by people from different backgrounds represent their own identities, and images and serve as memoranda for their descendants. Cross-cultural literature herein refers to literary works that are written in a specific cultural context that are different from an individual readers' cultural background. Research has been conducted on learning and understanding crosscultural literature for nearly half a century. With the ever-growing trend of globalization and modernization, it becomes more and more common to see teaching and learning practices on literature that are different from the teachers' and/or the students' cultural backgrounds. It is also prevalent to see the differences between the cultural background settings that contemporary teaching and learning practitioners are facing and their counterparts 50 years ago. Such differences became the first motivation for my revisiting the literacy learning theories that have been presented over the past 50 years and attempting to validate the feasibility of their application. More specifically, my particular research interest is: what teaching approaches serve to illustrate the authentic culture in the literary text and at the same time serve to help students improve their understanding of literature from a cultural perspective. Of equal importance one should see the generality that the term culture encompasses and consequently the wide scenario that cross-cultural literacy teaching and learning practices could apply. For example, a native English speaker learning Chinese literature or European literature might face cultural 
barriers since the culture s/he lives in is different from the specific cultural context within the text. To some extent, using literature representative of other cultures in the classroom typically brings new challenges for students.

An occasional chance of visiting a large, urban public high school in Miami, Florida provided me with a close look at such intercultural literacy teaching and learning in a $12^{\text {th }}$ Grade English class (many of the students in this school are of Hispanic background). The textbook presents students with a variety of literary theories which attempt to introduce the idea that the world is full of ideologies, theories, and biases. The course instructor told me that through applying literary theories, students could have more opportunities to interpret the literary texts with their own understanding and perspectives. Deeply impressed by such observations, I revisited the following related literacy learning theories:

1. Rosenblatt's (1968) reader-response (RRR) theory;

2. Reading comprehension research: cultural schemata (CS) theory;

3. Fish's (1982) interpretative communities (IC) theory;

4. Gunn's (1987) Cultural criticism (CC) theory.

In particular, the RRR theory considers the process of understanding a work as a recreation of it from the readers' (i.e., learners') perspective. It emphasizes the readers' effort from four respects: (a) readers should play an active role in the reading process; (b) readers need to bring to the work personal unique traits such as their own unique life experience, present needs and preoccupations; (c) the reading act for a reader is a process of construction, synthesis and then re-creation; and (d) the text meaning is manifested and enriched through a reader's interpretation. 
However, readers' active involvement in the interpretation of the literary texts connecting with their own life experience may prevent them from understanding the original meaning and the cultural contexts of literary texts. Barrera (1992) argued that a reader's meaning making for the literary texts is a culturally mediated process. From her perspective, "literacy and literature are cultural phenomena and are practiced differently across cultures" (p.232). From the outset, I specify the definition of culture as "the knowledge, ideas, beliefs, values, standards and sentiments prevalent in the group" (Fairchild, 1967, p.80). As different human groups have developed different cultural perspectives, reading the text is to read it in the light of one's culture. Bartlett (1932) pointed out one's cultural knowledge influences the interpretations of literary texts. By analyzing words and sentences within the text, readers may acquire meaning that is against their own personal knowledge of the world. One's personal knowledge is conditioned by one's culture such as occupation, sex, age, race, religion, nationality. Yu's (2005) study indicated when readers bring to the work personal unique traits, such as their own unique life experience, present needs and preoccupations, they sometimes have difficulties in understanding the ethnic identity and cultural distinctiveness in the text.

From the aforementioned literacy teaching and learning theories, it is obvious that an awareness of cultural differences does make a difference with respect to the learners' cross-cultural literature comprehension. However, the crucial reality is that the importance of culture is often neglected or given minimal consideration in practical literature classes.

Scholars have also put forward the concept of cultural schemata. Cultural schemata are conceptual structures that enable one to store information related to one's 
own culture in both conceptual and perceptual levels (Malcolm \& Sharifian, 2002).

Readers tend to use their cultural schemata when accessing literary texts. In the first step, readers may have some assumptions that are unique to a particular cultural group, such as rules, models, ideas, etc. Furthermore, with the common assumptions, members in the same cultural group tend to behave in the similar appropriate ways. Those assumptions are an integrated part of group members' perceptions or behavior patterns (Pritchard, 1990). Sometimes, individuals tend to internalize their cultural assumptions when they are reading a piece of literary work. In situations where the new information acquired does not match with readers' pre-existing cultural knowledge, the culture depicted in the literary text can easily be distorted. Therefore, it is of great importance for readers to have the relevant knowledge of culture depicted in the literature for the comprehension of the cultural referents within the selected texts while interpreting the literary works.

Clearly, an effective method for cross-cultural literature teaching and learning should not only help students identify different culture elements in the text but also overcomes the pre-existing cultural barriers that impede their understandings of the literary works. Cultural criticism (Gunn, 1987) is another critical lens through which any text can be viewed. It focuses on the elements of culture, such as different political beliefs, religions, ethnicities, and class and how they influence one's understandings of texts and perceptions of the world. The purpose of using cultural criticism in interpreting the literature is "to make connections between the literary text, the culture in which it emerged, and the cultures in which it is interpreted (Tyson, 2014, p.295). As a critical approach that considers the influence of the reader's cultural context, cultural criticism 
can be used in the classroom settings of cross-cultural literature teaching and learning in China.

\section{Statement of Problem}

China has the largest populations of English learners in the world (Crystal, 2008). Among these learners, the college undergraduate students-English majors and nonEnglish majors alike constitute the vast majority. English majors are usually at a higher level of ability of mastering the English language than non-English majors at the time of enrollment into the program. Having access to the best faculty assistance and English learning resources across the university, their English learning competencies have been further enhanced after two years of intensive English instruction in the English program. The higher language acquisition capability enables achievement of another teaching and learning objective: cross-cultural literature appreciation. In fact, English literature (it includes British literature and American literature) is mandatory for English majors at upper-intermediate and advanced levels at college. The purpose of teaching English literature is to enhance advanced Chinese English learners' language awareness and interpretative abilities to appreciate the literature by providing authentic English texts (Carter \& Long, 1990).

Traditional literature teaching in China use formalist criticism and biographical criticism ( $\mathrm{Li}, 1998)$. Formalist criticism focuses on the form, the nature of the genre, structural features, figurative language and images, and symbols of the literary texts, while biographical criticism concentrates on literary biography, the life of times of the authors that may include personal influences of the author, such as psychological and social factors, literary relationships, literary periods, and movements (Karolides, 2000). 
The two criticisms contend an objective and pre-determined nature of the meaning of literary texts. They advocate examining a text systematically, such as summarizing the main idea, identifying the themes of the reading piece, analyzing the main characters and events ( $\mathrm{Li}, 1998)$. With such an objective approach to the literary work in the literature classes for Chinese English majors, students are provided with an overview of text's historical context, an introduction of a writer's biographical information. Moreover, students are required to do close readings of an individual text with an emphasis on how the form, language, and literary technique all contribute to its meaning.

To some extent, formalist and biographical criticism use an objective approach focused on details about the author, historical context and literary mechanics to analyze literature. These two criticism guide students to understand the literature through comprehensive analysis of text-based information and identification of historical or bibliographical background in the literary works. However, these methods neglect the fact that literature comprehension involves readers' active participation, and they seldom consider the influence of reader's native culture in their reading process.

First, using the formalist or biographical criticism seldom invites students to be engaged in literature appreciation. The formalist and biographical criticism follow the following assumptions: (a) Understanding the author's intention and life experience is the key to understand the meaning of the text; (b) The meaning of literary texts is predetermined, objective and unique (Karolides, 2000); (c) Through objective analysis of the formal structures and techniques of the text, the meaning is manifested. Following these three assumptions, the formalist criticism and biographical criticism actually attempt to establish some universal principles or rules that could be applied in each 
setting for literature appreciation. "With the purpose of uncovering and identifying the common features of literature from different cultural traditions, the literature that defined by these criticisms is literature without the influence of the reader and the historical context" (Christenbury, 2000, p. 48). Influenced by the lens of two criticisms, in the literature class for Chinese English majors, literary appreciation is treated as a close, scientific and disinterested textual analysis rather than a cognitive process that requires students' active involvement. The students read the text for practical purpose: that is, knowing the content of the text, the author's identified themes and attitudes. Such reading has a touch of impersonality without personal involvement (Rosenblatt, 1968). Under these circumstances, students' roles are neglected or omitted; they are not given opportunities to be actively engaged in the literature appreciation process.

Second, formalist criticism and biographical criticism fail to provide students an opportunity to access cultural elements from the text. Literature opens a door for readers to see the world and construct identity from different perspectives; the readers of foreign language literature should be able to see diverse cultural elements from the literary texts and know the world in a totally different way. Understanding of the culture in the texts is very important for students' comprehension of the literary work. A culture informs readers about people's lifestyle, living conditions, communication and interaction in its literary texts. In general, the culture refers to "nation's civilization, psychological structure of the nation, spiritual pursuits, cultural customs, religion, history, economy, political system and other aspects of ideology from different facets" (Zhen, 2012, p.36). understanding is a process of connecting discrete bits of information and carrying constructed knowledge into diverse contexts and circumstances (Goodman, 1982). To 
have a better understanding of the literature, it is necessary to acquire some cultural awareness with respect to one or more facets in the above definition of culture. As formalist criticism and biographical criticism discourage acquisition of cultural knowledge, students may not be able to develop sufficient cultural awareness, hence the typical Chinese instruction of literature impedes appreciation of that literature.

Third, the formalist and biographical approaches to literary criticism neglect the fact that the native culture brought by the readers unavoidably influences their appreciation of a cross-cultural literature text. Herein native culture refers to the social environment which the reader grows up in or is familiar of. It is differentiated from the cultural elements in the literary text that represents another culture. As literature comprehension is a cognitive process that requires readers' response, the native culture that pre-exists in the readers' mind gets unavoidably involved in the conceptualization, rationalization, inference and analogy of whatever the text might cover. However, the native culture where the text interpretation is impacted often differs significantly from the cultural phenomenon expressed in the texts. This mismatch unfortunately often becomes one of the impediments to understanding the literature (Gatbonton \&Tucker, 1971; Steffensen, Joag-dev \& Anderson, 1979). The influence from native culture has been generalized as the "culture schemata" (Cook, 1994). It indicates the undesired interaction of readers' native culture in the process of their appreciating a different cultural representation. More details about the theory of culture schemata can be found in the section of literature review.

In the case of traditional English literature curriculum and instruction, the Chinese students will be passively under the influence of the cultural schema from their own 
Chinese (unified or diversified) culture during any attempt at cross-cultural text interpretation. The cultural stumbling blocks that Chinese English majors are confronted with are rarely addressed neither by the formalism or the biographical criticism. In other words, those traditional methods ignore the cultural schemata's influence on students during the reading process.

\section{Purpose of Study}

The purpose of this study was to examine the effect of utilizing a cultural criticism approach to teach English literature to Chinese undergraduate English majors. More specifically, the study was undertaken to understand the difference two different literary teaching methods (Using cultural criticism and using a traditional formalist and biographical approach) might make on students' cultural understandings of the literary texts and on their literature comprehension.

Herein cultural understandings of literature refer to awareness of cultural influences in literary texts from cultural perspectives. Literature comprehension refers to "the ability to connect discrete bits of information from a text in order to construct meaning" (Goodman, 1982, p.52). Literature comprehension is an act of understanding literary works including context, themes, and textual meaning.

\section{Research Questions}

In order to investigate the effect of using cultural criticism for Chinese English majors in China, this research addressed research questions regarding whether Chinese English majors at college level would benefit by using cultural criticism to learn English literature. The focus of this study tested the assumption that using a cultural criticism 
approach could be more effective than a traditional formalist and biographical approach in terms of cultural understanding and comprehension of the British literature texts.

Specifically, two main research questions were addressed:

1. Does a cultural criticism approach result in better cultural understanding of literary texts than a traditional formalist and biographical approach?

2. Does a cultural criticism approach result in better literature comprehension than a traditional formalist and biographical approach?

The researcher was also interested in understanding the difference of a cultural criticism approach taught by the same instructor at different levels of proficiency. Specifically, the following research questions were addressed:

3. Does a cultural criticism approach taught first result in better cultural understanding of literary texts than a cultural criticism approach taught second by the same instructor?

4. Does a cultural criticism approach taught first result in better literature comprehension than a cultural criticism approach taught second by the same instructor?

\section{Significance of the Study}

With the ever-growing trend of globalization, cross-cultural communication has become increasingly common. Correspondingly, in order to enhance mutual understanding and bridge the cultural barrier, more effective cross-cultural literature teaching and learning is demanded. Seen from the section above that discussed the purpose of the study, this study was undertaken to investigate the effect of employing 
cultural criticism in English literature teaching and learning for Chinese English majors. The significance of the study could be summarized in the following two aspects:

First, theoretically, the study validated Gunn's cultural criticism theory and Rosenblatt's reader-response theory in the context of literature education. In addition, it explored a new pedagogy developed creatively by the researcher for cross-cultural literature teaching, which integrated Rosenblatt's reader-response theory, Cook's cultural schemata theory, Fish's interpretative communities theory and Gunn's cultural criticism theory. It also provided comparisons between teaching methods that use cultural criticism and biographical/formalist criticism. The comparisons focused on the effectiveness and the applicable domain regarding employing different teaching methods on cross-cultural literature teaching and learning.

Second, practically, it was the first time that a pedagogy of cultural criticism was applied to the settings of English literature teaching and learning in the higher education domain in China. This included the curriculum design, instruction and implementation as well as performance evaluation. The study enriched the teaching methodologies and practices, which guided Chinese English majors to explore a multiplicity of cultural perspectives and participate in cultural exchanges actively in the cross-cultural literature appreciation. The research provided empirical data for comparing the effect of various literature teaching methodologies. It also served as reference for future implementation of cultural criticism in the cross-cultural literature education. 


\section{Delimitations}

This study was delimited to undergraduate junior-year English majors in four British literature classes at the Department of English at Anhui Agricultural University, China.

\section{Operational Definitions}

The following terms have been defined to clarify their use in the current study:

English as a foreign language (EFL). It is a class usually taught in counties where English is not a medium of instruction but is learned at school. In this context, the target language is not commonly used in the community and there is little opportunity to experience the language outside of class (Oxford, 2001).

English language learner. It is an active learner of the English language who may benefit from various types of language support programs (NCTE, 2000).

Chinese college English majors. These are Chinese students whose specialization is English at undergraduate level (Hu, 2004).

Cultural Schemata Theory. People understand the new experience through retrieving memory from their previous experience; the interpretation of the new experience could either conform to a stereotypical version or deviate from it (Cook, 1994).

Culture. According to Tyson (2004), "a culture is a collection of interactive cultures, each of which is growing and changing, each of which is constituted at any given moment in time by the intersection of gender, race, ethnicity, socioeconomic class, occupation, and similar factors that contribute to the experience of its members " (p.294). 
Cultural Criticism. Focuses on the elements of culture and how they affect one's perceptions and understanding of texts. This form of criticism examines how different religions, ethnicities, class identifications, political beliefs, and views influence the ways in which texts are created and interpreted (Gunn, 1987).

Curriculum. According to Hass (1978), it refers to: “(a) A school's written courses of study and other curriculum materials; (b) The subject matter taught to the students; (c) The course offered in a school; (d) The planned experiences of the learners under the guidance of the school" (p. 4). All four aspects mentioned above were included in the English curriculum used in this research.

Pedagogy. According to Simon (1992), "the integration in practice of particular curriculum content and design, classroom strategies and techniques, and evaluation, purpose, and methods" (p.262).

Culture awareness. Cultural self-awareness includes "recognition of one's own cultural influences upon values, beliefs, and judgments, as well as the influences derived from one's work culture" (Winkelman, 2005, p. 9).

Cultural understanding of literary texts. It refers to understandings of the cultural influences in literature texts from the general concept of culture, such as "a nation's civilization, psychological structures of the nation, spiritual pursuits, cultural customs, religion, history, economy, political system and other aspects of ideology from different facet" (Zhen, 2012, p.36). In the study, the cultural understanding of literature texts was measured by the five cultural components: customs and beliefs, economic status, politics, gender, as well as free will in marriage as assessed in two cultural analysis essays. 
Literature appreciation. It refers to "the evaluation of works of literature as an academic and intellectual exercise. It is the process by which the recipient of a work of literature acquires an understanding of its theme(s) and subject matter, and obtains insights into the ways in which its formal structure helps realize them" (Olufunwa, 2001, p.350).

Literature comprehension. One's ability to connect discrete bits of information from a text in order to construct meaning (Goodman, 1982). Literature comprehension is an act of understanding literary works including context, themes, and textual meaning In this study, literature comprehension was measured by three subcategories in three British literature comprehension tests: (1) cultural and historical context; (2) identification of themes, purpose and plot developments; (3) literature analysis (literary analysis is an approach to understand the textual meaning through analyzing plot/structure, character, setting of the literary work). 


\section{CHAPTER II}

\section{LITERATURE REVIEW}

The following literature review section examines seven components of research related to my study: (a) Theoretical framework; (b) Application of related theories in the classroom settings; (c) Views of curriculum; (d) English literature curriculum in China; (e) Definition of Culture; (f) Relationship among language, literature, and culture; (g) Pedagogical issues and suggestion in literature class. Key concepts regarding readerresponse theory, culture schemata theory and, theory of interpretative communities, and theory of culture criticisms comprise the theoretical framework for the current research.

\section{Theoretical Framework}

The theoretical framework that I used for the research was derived from the following theories:

1. Rosenblatt's (1968) reader-response (RR) theory

2. Reading comprehension research: cultural schemata (CS) theory

3. Fish's (1982) interpretative communities (IC) theory

4. Gunn's (1987) Cultural criticism (CC) theory

With the purpose of developing a possible literary appreciation approach for Chinese undergraduate English majors in the cross-cultural literature teaching and learning setting, the aforementioned four theories comprise the theoretical framework. In the study, the RR theory invited students' responses with literary texts; the CS theory explored how students' pre-existing knowledge impedes their understanding of the texts; the IC theory encouraged students from different cultural background to share their diverse perspectives in a literature learning community; and finally the $\mathrm{CC}$ theory helped 
students identify different cultural elements in literature texts as well as facilitate their cross-cultural understanding. The following literature review section provides an overview of each theory respectively.

\section{Reader-Response Theory}

According to Rosenblatt (1968), the relationship between the text and the reader is fundamental to having aesthetic experiences. Aesthetic reading involves readers' social and psychological insights, and the human concerns embodied in the aesthetic experience (Connell, 2000). When the readers adopt an aesthetic stance, they pay close attention to the relationship between their past experience and a particular text. Their feelings are trigged by particular words in a particular text and then they appreciate the texts with emotions, senses, and their previous living experience. Further, Rosenblatt (1968) contended that a dynamic meaning of the text which calls for readers' engagement by regulating what enters their consciousness. In brief, the aesthetic nature of reading requires readers to play an active role in the process of constructing the meaning from a text.

Because of the aesthetic nature of the reading process, the meaning of the text is no longer a predefined objective item. The text is no longer perceived as "an independent entity" (Karolides, 2000, p.16). Its interpretation is no longer unique but a dynamic process along with different readers' engagement; and thus, readers are required to be aware that "what literature means" (Rosenblatt, 1968) counts more than "what literature does" (Rosenblatt, 1968) in interpreting the texts.

Rosenblatt (1968) argued that the reader plays an active role in the reading process. The process of understanding a work "implies a re-creation of it, an attempt to 
grasp completely the structured sensations and concepts through which the author seeks to convey the quality of his sense of life" (Rosenblatt, 1968, p. 113). That being said, the reading act for a reader is a process of construction, synthesis and then re-creation based on his/her experience of the text. Meaning is manifested and enriched through the reader's interpretation. Nevertheless, overemphasis on interpretation of texts from readers' perspectives integrating with their own life experience, may restrict them into a narrow scope; it could even prevent readers from understanding the cultural distinctiveness and cultural diversity within the text (Yu, 2005).

In Rosenblatt's (1968) view, the literary experience could be viewed as a transaction between the reader and the text. The reader and text continuously influence each other and benefit each other mutually, that makes each transaction as a unique experience. The text itself no longer plays the dominant role in one's reading process as argued by the New Critics. The readers' own interpretation towards the texts integrated with their lived experience assists them to reconstructing the meaning of the text. Transactional theory, as argued by Rosenblatt (1981), points to the interrelationship between the knower and what is already known. With regard to literary reading, Rosenblatt (1981) claimed that the position of the knower and the known are ultimately changed during the course of knowing. According to Rosenblatt (1981), transaction occurs as an ongoing process in which "the elements or parts are seen as aspects or phases of a total situation" (p.35).

In a sense, the text gains its full meaning with readers' living through experience, and the readers derive the knowledge and then enrich their understanding under the direction of the text. In contrast with New Criticism Theories which focuses on "what the 
text tells", Rosenblatt's reader-response theory (1968) advocated readers' own experiences should be emphasized. It is necessary for readers to bring their individual background knowledge, beliefs, cultural referents and cultural context into the reading act. To some extent, the reading act for a reader is a process of construction, synthesis and then re-creation integrating with a reader's life experience. As readers experience in life and reading influence their responses to the word and the world in which they live, the text meaning is manifested and enriched through recursive and cumulative aesthetic reading (Rosenblatt, 1968).

\section{Cultural Schemata Theory}

According to Rumelhart (1980), schema refers to "a hypothetical mental structure for representing generic concepts stored in memory" (p.34). Schemata theory originated from the Gestalt psychology school during the 1920s and 1930s. Basically, it claims that people understand the new experience through retrieving memory from their previous experience; the interpretation of the new experience could either conform to a stereotypical version or deviate from it (Cook, 1994). According to Suliman (1980), meaning is not a characteristic of texts. Rather, texts are constructed by authors and then they are comprehended by the readers. Meaning is validated by both the author and reader. Although the text has the potential to convey meaning, it does not embody meaning in itself. Since not all information is demonstrated within the text, the role of prior knowledge of the reader in reading comprehension is critical. In this view, Suliman (1980) argued that a complete understanding of the texts depends on the way in which the writer constructs the text and the way the reader reconstructs and constructs meaning. The writers' schemata are established from their own values, their concepts, and their 
experiences. Therefore, the text reflects what belief the writer has, as well as what the writer is trying to communicate. Because comprehension results from reader-text transaction, what the reader knows, who the reader is, what values guide the reader, and what purposes or interests the reader has will play in the reading process (Goodman, 1994). Rumelhart (1981) further stated that schemata represent knowledge at all levels of abstraction. Whereas all readers share some knowledge, it is culturally specific knowledge that differentiates cultural groups. In the process of reading, the textual information interacts with the reader's personal knowledge of the world, which in turn is conditioned by age, sex, race, religion, nationality, occupation--in short, his or her culture (Anderson, Reynolds, Steffensen, \& Taylor, 1982).

Reading comprehension as affected by pre-existing knowledge has been studied (Gatbonton \&Tucker, 1971, Steffensen, Joag-Dev \& Anderson, 1979; Spiro, 1977; Prichard, 1990). These studies found that schemata significantly affected reading comprehension. A study conducted by Gatbonton and Tucker (1971) showed that EFL students drew incorrect assumptions when reading unfamiliar texts because of cultural misunderstandings; however, when provided with pertinent cultural information, their performance increased significantly. Prichard (1990) demonstrated that the cultural origin of the text has a greater effect on ESL reading comprehension than does linguistic complexity. A study conducted by Steffensen, Joag-Dev and Anderson (1979) indicated that participants could have more culturally appropriate elaborations of the native passage when provided them with a text written about a wedding in their own culture, while they could have more cultural distortions of the text that is different from their culture.

Steffensen et al. pointed out that exposing participants to culturally unfamiliar texts could 
be regarded as an outright intrusion on one's own culture. According to schemata theory, elaborations may be produced when a text is incomplete and distortions may be developed when the reader's schema diverges from the schema presupposed by the text. The inferential processes that produce elaborations and distortions may be active when the passage is read, or later when the material is recalled (Spiro, 1977). Barlett (1932) provided examples from the protocols produced by educated English men attempting to recall the North American Indian folktale The War of the Ghosts. The subjects typically modified the tale in a manner consistent with their own culture.

\section{Theory of Interpretive Communities}

Fish's (1982) interpretive communities theory examined how the interpretation of a text relies on each reader's own subjective experience within one or more communities. From the perspectives of Fish (1982), the readings of a text are culturally constructed. This cultural context often includes authorial intent, though it is not limited to it. Fish (1982) further claimed that when readers are interpreting the fictive texts, they are part of an interpretive community that gives them a particular way of reading a text. Any act of communication requires interpretations from the interpretative community. To some extent, the authority for the interpretative process is attributed to the interpretative community that one belongs to; neither the author nor the reader could play the important role as the interpretative community does during this process.

\section{Theory of Culture Criticism}

Cultural criticism (Gunn, 1987) focused on the elements of culture and how they influence one's perceptions and understanding of texts. According to Berger (1995), "cultural criticism is not just about art and literature, but about the role that culture, in 
both aesthetic and anthropological senses, plays in the role that we now see is increasingly important not only for what it reveals about our social, economic, and political institutions, and also for how it shapes these institutions and our consciousness" (p.38). Cultural criticism examined how different religions, ethnicities, class identifications, political beliefs, and views influence the ways in which texts are created and interpreted (Gunn, 1987).

Cultural criticism is an activity that "involves literary and aesthetic theory and criticism, philosophical thought, media analysis, popular cultural criticism, interpretive theories and disciplines (semiotics, psychoanalytic theory, Marxist theory, sociological and anthropological theory, and so on), communication studies, mass media research, and various other means of making sense of contemporary (and not so contemporary) culture and society" (Berger, 1995, p,2). "Cultural criticism is not just about art and literature, but about the role that culture, in both the aesthetic and anthropological sense, plays in the scheme of things-a role that we now see is increasingly important not only for what it reveals about our social, economic, and political institutions, but also for how it shapes these institutions and our consciousness" (Berger, 1995, p.38).

Appreciating and understanding the literature requires readers to understand the relationship of works of art to culture and of cultural matters to society and politics (Berger, 1995). Employing cultural criticism emphasizes the integration of the culture of literary works, even the most canonical, with the whole range of cultural expression (Fiedler, Terence \& Triandis, 1971). In the classroom, using cultural criticism calls for up-to-date and engaging thematic curriculum where culture, social structures, and historical circumstances are explored by side by side with a particular emphasis on those 
issues affecting real people in the present day. While it draws on the insights and interests of "multiculturalism," cultural study is both broader in its inclusion of issues of social class, women's studies, and popular culture, and more critical in the emphasis on social change (Fiedler, Terence \& Triandis, 1971). Thus, the cultural criticism studies explore not only the high literary culture that has been the traditional domain of English teaching, but also the lives of people whose voices, perspectives, and experiences are seen as the very stuff of which culture is made.

Employing cultural criticism in literature class invites a wide variety of new and potentially invigorating writing into teaching, such as interviews, ethnography, testimonials, surveys, film, and media analysis. It urges students to be self-reflective but not cavalier about the disciplines we work in. While mixing genres and crossing disciplinary boundaries, cultural criticism spurs students also to consider how the establishment of genres and disciplines has functioned historically.

\section{Distinction between acquiring cultural knowledge and engaging in cultural}

criticism. Acquiring cultural knowledge assists students in contextualizing the literature. According to Kentner (2005), traditional culture teaching focuses on information-oriented cultural elements, such as "the customs, knowledge, morals, practices, skills, music, literature, art, behaviors, languages, family, religions, signs, symbols, etc. of generations and communities/countries" (p.9), which may be imparted to learners directly. The teaching method focuses on the transmission of factual cultural information to learners (Tahnasoulas, 2001). Learners are required to acquire cultural knowledge by gathering, organizing and reporting cultural related information in the literary texts. Kostelníková (2001) pointed out the following aspects of traditional culture teaching and learning: “(a) 
Literary texts serve as a source of information about the culture of the target language; (b) The cultural information acquisition usually takes the format where teachers raise questions (e.g. the most commonly used multiple-choice questions) and students find the answer from the text. (c) It provides explanations, glosses, brief cultural information in a note or gloss. (d) It provides cultural background information such as reading or listening comprehension" (p.18). As the traditional approach concentrates on the factual information in the texts, it may diminish the capacity of literature to portray and enliven the human experience for readers.

Engaging in cultural criticism arouses learners' cultural awareness through the act of inquiry. Prior to understanding the foreign culture within the texts, learners need to understand their own cultures first by reviewing their own frames of reference; then they are required to compare or contrast the unfamiliar culture with their own cultural context (Kentner, 2005), and thus learners experience multiple contexts as a means of reflecting on the complexity of the world. It involves learners' actively constructing a sense of other's culture, comprehending the culture depicted in the text, reflecting on it, reshaping the worldviews towards the foreign culture, and identifying the gaps between what learners already know and the targeted culture.

On the whole, during cultural criticism engagement, learners are able to know not only what the cultural knowledge is, but also how to engage in cross-culture practice. Through cross-cultural practice, learners may also discern the difference in the values, beliefs, and attitudes among different cultures (Moran, 2001). 
To sum up, cultural knowledge transferring is part of the culture criticism engagement but is not the main purpose. The latter emphasizes the process where the transferred cultural awareness contributes to the understanding of texts.

\section{Rationale on Connecting with Four Theories as a Theoretical Framework}

The reader-response theory considered understanding a work as a process of a reader's active recreation of a text by integrating their own perspectives (Rosenblatt, 1968). This study attempted to apply the RR theory and measure the literacy reading comprehension from Chinese undergraduate English majors based on their reading responses.

The cultural schemata theory viewed that the literary text may not be understood by the reader if the information acquired from the literature works does not match the reader's pre-existing knowledge (Pritchard, 1990). In the present study, the participants were Chinese English majors and they were required to read and understand some British literary works. By default, the students' cultural schemata come from their Chinese cultural background, which might add some difficulties to their reading comprehension. Applying the CS theory, this study aimed at exploring the conditions where the cultural schemata impeded the text understanding and how differently the influences were.

With the aforementioned research hunch, Fish's (1982) interpretative communities theory was further reviewed. Fish (1982) argued that a text's meaning for a reader emerges from an interpretive community. In the present study, a group of Chinese undergraduate English majors was viewed as an interpretative community. The present study paid attention to these Chinese English majors' responses to literature from cultures other than their own in their literature learning community. 
Cultural criticism theory examined how the elements of culture influence one's perceptions and understanding of texts. (Gunn, 1987). In an attempt to help Chinese English majors develop cultural understandings of literary texts from cultures other than their own and integrate their own cultural perspectives into the literature comprehension, a reader-response based cultural criticism approach was developed for teaching literature in such a cross-cultural context. Using a cultural criticism approach may bring the awareness of British cultural traditions to the Chinese English majors in the context of cross-cultural literature teaching.

On the basis of Rosenblatt's(1968) reader-response theory, cultural schemata theory, Fish's (1982) interpretative communities theory and Gunn (1987)'s cultural criticism theory, the present study proposed a teaching model focusing on reader-centered cultural criticism that English teachers could employ in teaching British literature for Chinese English majors. The literary instruction model could be a subject of critical and social as well as literary inquiry, rather than an exercise in close reading of texts that remain irrelevant to students' experience. To be specific, it followed six steps: (a) teachers activate students' prior knowledge of literature in general. Students are highly encouraged to connect the novels with their own experience, and then identity the universal themes that the literary pieces embody. At this stage, although they are exposed to the text that the culture is different from their own, the reader-text transaction will be realized through activating their prior knowledge; (b) teaching culture-related knowledge within the literary text. The culturally relevant knowledge for British literature could be explanations of allusions and reference loaded with cultural meaning, and historical and social backgrounds of the novel. For example, British politeness, religious beliefs, and 
tradition of nobility in British society. (c) request for readers' response for the culturerelated features of the text. Each learner identifies the most striking point that they have learned from the cultural elements with the text and respond the text from their perspective; (d) sharing with the interpretative community. (e) using the cultural criticism to teach critical textual analysis. The teachers assist students to have critical sense of literary conceptions. (f) making comparisons between two different cultures. At this stage, students reflect upon their own literary traditions and bridge the gap between their previous cultural knowledge and the culturally relevant knowledge with the literary text.

All these theories emphasize the nature of interpretation, rather than the definitive nature of constructive meaning (Rogers, 1997). Therefore, they could be integrated into the present study. As the conceptual framework, these theories helped achieve the following goals in the teaching model: (a) assisting students in the study to create a new critical perspective that incorporates the text-centered and teacher-led orientations to literature instruction; (b) ensuring a reader-response orientation that encourages students in the study to join a classroom community where readers' knowledge and experience are valued dimensions of the reading experience; (c) using cultural criticism that challenges and critiques students' received ways of seeing in the literary texts in the study.

\section{Applications of Related Theories in the Classroom Settings}

\section{Application of Reader-response Theory}

Malo-Juvera's (2014) study investigated whether the instructional literary unit of the young adult novel Speak using reader-response theory would diminish the efficacy in terms of adolescents' rape myth acceptance. Following reader-response theory (Rosenblatt, 1978) that posited the meaning of the text is constructed in the literary 
transaction between the reader and the text, Malo-Juvera (2014) designed a reader response unit on Speak. In this unit, group discussions and writing were included. After small group discussions centering on four different essential questions about the rape experience of the characters, students were required to write up four pieces of individual reader-response papers. After that, students also had a group discussion. According to Malo-juvera (2014), "reader response-based instruction attempts to engage students by using texts that have connections to the students' lives, by using an inductive approach, and by having discussions that encourage students to ask questions" (p.415).

The results of the study showed that students' engagement in the reader responsebased dialogic instruction could lower adolescents' rape myth acceptance. Since it is a quantitative study, further research can also identify how the reader-response approaches help students validate the meaning making process between the word and the world from a qualitative perspective.

\section{Application of Interpretative Communities Theory}

In the dissertation, Teaching culture through language and literature: the intersection of language ideology and aesthetic judgment, Rojas-Rimachi (2011) studied how the learning of Spanish literary language reshapes students' minds in the context of a mainstream English culture. It applied interpretative community theory to help learners coming from a main stream English culture readjust their values and perception towards the culture in the Spanish literary works. Specifically, each participant in the study gave a presentation on some literary topic. Group discussions were also held where the participants exchange of different perspectives on the presented Spanish literary pieces. The presentation and group discussions provided the students with a space for enlarging, 
modifying, revisiting and re-elaborating old information with new nuances coming from the exchange of opinions and perceptions on certain matters. Rojas-Rimachi (2011) argued that students from the English culture were able to rethink and bridge their native culture with the foreign one, in the situation where they progressively make the foreign one part of their daily life through dialogue. From the author's perspective, literature, as a fundamental teaching and learning tool, allows students to access the different aspects of cultural learning. In order to assist students to understand the nuances of classroom culture in Spanish literary texts, Rojas-Rimachi (2011) also suggested that both teachers and learner in the learning community must share their own perspectives to construct a culture. Influenced by the lens of interpretative community theory, the study emphasized in the cross-culture literary context, a shared dialogue in the learning community is required between students and teachers so as to construct a new space for understanding a foreign culture. Although the study acknowledged the significance of dialogue in literature instruction, it lacked detailed descriptions of classes where teachers and students apply the dialogic approach for literature engagement to explore the culture, social structures, and historical circumstance. Since the details might help other researchers in evaluating the applicability of implementing the same approach in their own classrooms, it might be a good next step to collect the details such as class organization, choices of literary pieces as well as the proportion of the presentations and discussions in each class.

\section{Application of Cultural Schemata Theory}

Dehghan and Sadighi (2011)'s study explored the effect of cultural schemata on the Iranian EFL learners' reading performance in bottom-up processing (word 
recognition, literal comprehension and finding referents of pronouns) and top-town processing (making inferences, etc). A total number of sixty-six female pre-university students participated in the study. The study use multiple-choices questions as the instrument to test student participants' reading comprehension on the five culturally familiar literary texts and another five culturally unfamiliar literature texts. The culturally familiar literary texts included the topics that are part of the cultural schema of this group of participants, such as Nowrooz, Hafez, Persepoilis, Persian wedding ceremonies and Ramadan fasting. The culturally unfamiliar texts covered the topics about Halloween, William Blake, Roman Colosseum, Western wedding ceremonies, and Independence Day. The study showed that the level of text familiarity significantly influenced students' overall performance on reading. The students performed better on local items compared with global items both for both texts.

To some extent, the study confirmed the validity of cultural schema on reading comprehension. That being said, when the students are familiar with cultural norms, they are more likely to have a better understanding of the text. The study showed that one's familiarity with culturally-oriented topics could be helpful in the reading comprehension. Yet the study did not point out the barriers that the students are confronted influenced by the cultural schema during reading the culturally unfamiliar texts. Furthermore, the study did not explain why the students tend to refer to their own cultural features in cases of unfamiliar norms. Future studies could be conducted to address such issues.

\section{Application of Cultural Criticism Theory}

Hua and Alsup (2010) investigated teachers' perceptions and instructional practice of teaching young adult literature in classrooms at secondary level in the rural 
Midwestern region in the U.S. The study (Hua and Alsup, 2010) described the struggles that teachers had when integrating multicultural young adult literature into their largely homogenous classrooms including White, middle class, American-born students. Hua and Alsup (2010) attempted to address the concerns of teachers regarding how they can guide students to explore the cultural differences in diverse texts and provide the possible approaches to help students discuss the differences of culture, and therefore enhance cross-cultural understanding. With vivid descriptions on how two pieces of young adult literary works, Yang the Youngest and His Terrible Ear and American Born Chinese were used in a high school ESOL class including students from different countries. The study identified theoretically and philosophically consistent pedagogical strategies related to the theory of cultural criticism in the classroom setting. That being said, under the guidance of teachers, students were engaged in examination of their cultural background, discussion of cultural differences, analyzing cultural features of the text and the difficulties they might encounter as cultural outsiders while reading two pieces of literary works. Hua and Alsup (2010) concluded that using a reader-centered cultural criticism approach helped students challenge and criticize the received ways of seeing in the literary texts. Yet Hua and Alsup (2010) did not further discuss teachers' conceptions of multiplicity and diversity issuing from literature classrooms. Further research can investigate more about how the teachers value texts in all their multiplicities.

\section{Views of Curriculum}

Hass (1978) listed four tentative definitions of curriculum. According to Hass (1978), the term "curriculum” has been used to mean: “(a) a school's written courses of study and other curriculum materials; (b) the subject matter taught to the students; (c) the 
courses offered in a school; (d) the planned experiences of the learners under the guidance of the school" (p. 4).

Furthermore, Hass (1978) argued that curriculum should have a set of broad goals incorporating related specific objectives that is planned in terms of a framework of theory and research or past and present professional practice, which allows individual learners to have all the experiences in a program of education. Basically, the curriculum should illustrate the core concepts of the disciplines explicitly, clarify the primary objectives of the disciplines seriously, and identify the future direction of the disciplines with insightful perspective.

In conventional views, curriculum in English Language Arts touches upon three aspects of English study: (a) knowledge about the language (grammar); (b) facility in using the language (composition); (c) appreciation for the language (literature) (Hodges, 1980). According to Tchudi (1991), "the curriculum in English Language Arts, hefty as it was, turned out to be a laminate of three interrelated traditions: language, literature and composition. Tchudi (1991) further pointed out that "these three components share a broad common approach to pedagogy: they were "knowledge" or "content" centered" (p.5). It required teachers to present knowledge concerning the language, writing, and literature, and then ask students to apply the knowledge they have learned in class. Tiedt (1983) posited that the content of the curriculum in English Language Arts includes three aspects: the English language, literature concepts, and the language skills. Specifically, with regards to the English language and the language skills, students are required to study speaking and writing about language as well as listening to language and reading the many books about language. Additionally, students are required to have access to a 
variety of literary forms, such as poetry, fiction, nonfiction, drama, biography, etc. (Tiedt 1983).

The essential components in English Language Arts curriculum, English curriculum has two major dimensions: one is to provide basic literacy, general knowledge in the English discipline, and skills in language acquisition for all the learners; the other is to actualize personal growth, talent development, competence in communication and thinking in accordance with the noblest humanistic values of a free and open society.

Curriculum and instruction in English literature usually follow the traditional language-based approaches (Li, 1998; Carter and Long, 1990, Akyel and Yalcin, 1990) where literature is used as the medium to teach English language under the guidance of the language-based approach; Carter and Long (1990) stated that literature and languages are integrated into the English language teaching that are supplementary with each other. Akyel and Yalchin (1990) stated that literature plays an important role for students to expand language awareness as well as cultural understanding.

Hansen (2008) have suggested that the literature curriculum needs to cultivate students to have a "critical openness to the world with a critical loyalty toward the local" through the encouragement of dialogue and respect for other people and their traditions (p.8, cited by Choo, 2011). Choo (2011) regarded it as a "Cosmopolitan Literature Curriculum" (p.15). From Choo (2011)'s perspective, one may start perceiving the world through the lens of one's culture and one's own traditions; under the influences of these two interrelated driving forces (culture and tradition), one could ultimately realize transformation so as to be more open to the surrounding world. Choo (2011) also have pointed out that utilitarian and transcendental values are included in the cosmopolitan 
literature curriculum. With such values, students could be able to "shift among different communities, and to relate and communicate with diverse groups by having dispositions related to cosmopolitan curiosity, openness, and empathy toward others" (p.15).

\section{English Literature Curriculum in China}

The English literature course, as one important component in English language teaching, has opened for English majors at their upper-intermediate and advanced levels for many years at the college level (Li, 1998). It is regarded as a "reinforcement device" (Du, 1993, p.168) that helps to facilitate students' English language learning. Literature Curriculum for English majors in China has two main goals: one is about language acquisition and the other about literature appreciation (Zhen, 2012). Zhen (2012) viewed the emphasis on English literature curriculum at the college level is designed to assist English majors in development of integrated skills of English in order to play a more active role in international communication.

Although the English literature teaching in China has also adopted the traditional language-based approaches in a broader sense, its curriculum setting has another emphasis on the literary history. This is firstly reflected in the textbook organization. In China, each English department has much freedom to select the textbooks for students, and then design its own curriculum. Although the textbooks vary, the selected influential literary works are often chronologically organized based on the literary historical period. One of the distinguishing features of the textbook is that it integrates both literary history and literary works (Lu and Jin, 2013). For example, one of the textbooks edited by Peilan Wang (1999) put English literary history into seven literary periods: The English renaissance, the period of revolution and restoration, the age of enlightenment, the 
romantic period, the Victorian age, modernism and post-modernism. After giving brief background information on the historical, cultural and social/cultural background of a particular literary period; it then reviews the classical writers' bibliography and representative works. Following that, excerpts of the author's most influential works are presented (Liu, 2013). English literature teaching in China provides students an overview of historical and biographical backgrounds of literary works. It emphasizes linguistic and literary analysis particularly $(\mathrm{Li}, 1998)$. Normally, during the class, students are guided to analyze the theme, viewpoints of the writer, plot development, symbols, and characters. A typical scenario in the English classroom is simply students copying the notes that the teacher presented. To some extent, teachers play the role as the authority in the literature class. Moreover, besides reading the selected excerpts in the textbook, students have little opportunities of access to more resources and materials related to the literary works. They are forced to learn literature and remember the summary of classical works in certain literary historical period. From the perspective of Li (1998), "literature textbook in China is the over-emphasis on literary history rather than on literary works" (p.9). A list of names of representative authors, literary works and the social and political situation of each literary period are required for students to remember, yet the original pieces have rarely been read $(\mathrm{Li}, 1998)$. Even if students read a few selected excerpts of the work under the guidance of the teacher, they fail to have a comprehensive understanding of the literary texts. Zhen (2012) attributed it to the fact that "English learners in the EFL context lack necessary knowledge of English culture" (p.38). Having different cultural background, students may have various interpretations and responses to the views of the author (Zhen, 2012). Zhen (2012) further argued that without accumulating related 
information regarding the cultural background of English, reading the classical English literature such as Shakespeare, Charles Dickens, Jane Austin could be a challenging task for the students, even if English majors had a good command of English language. The difficulties that they are confronted with are various. On the whole, the impeding factors for them are as follows: (a) the literary conventions they have access to in the American literature are different from their own; (b) the influence of culture schemata; (c) students' insufficient understanding of the socio-cultural and political factors in the text.

\section{Definition of Culture}

Different scholars have different understandings of culture. According to Tapp (2007), "culture is a set of learned beliefs and behaviors shaping how members view and experience the world" (p. 45). Robbins, Fantone, Hermann, Alexander, \& Zweifler (1998) stated that individuals bring their cultures of affiliation. In these scholars' perspective, cultures of affiliation may include in part religious groups, ethnic groups, social classes, and voluntary and professional organizations they have come to embrace (Robbins et al.,1998). According to Philipsen (1987), “a culture can be viewed from many perspectives, each of which provides one partial but important glance at the nature of things cultural" (p.76). In Goodenough's (1964) views, culture does not consist of things, people, behavior, or emotions. It is rather an organization of these things. It is the forms of things that people have in mind, their models for perceiving, relating, and otherwise interpreting them. Robinson (1985) viewed cultural understanding as “an ongoing, dynamic process in which learners continually synthesize cultural inputs with their own past and present experience in order to create meaning - a synthesis between 
the learner's home culture, the target culture input, and the learner as an individual" (pp. 11-12).

From the definition cited above, different scholars defined culture in different perspectives. However, the common ground of their definition is based on associating culture with communication. Different theories of culture indicate that culture shapes individuals' understanding of literary texts.

\section{Relationship among Literacy, Literature and Culture}

There is a close relationship between literature, language, and literacy. As Moody (1967) demonstrated, "the study of literature is fundamentally a study of language in operation" (p. 22). Each literary work is essentially the collection of words that are permanently available for the student to inspect, to investigate, to analyze, and to build together (Moody, 1967). The inclusion of literature in the curriculum helps train students in the skills of reading, writing, and thinking, because literary works incorporate so many complex language structures, skillful writing styles, intricate social and cultural contexts, and deep portrayals of reality based on authors' observation, reflection, and recreation of the subjects that they are confronted with. The more a person reads, the more knowledge he or she gains in reading, writing, and thinking. Therefore, experiencing a work of literature for the student is an intellectual process of acquiring knowledge and developing critical thinking from text (Peregoy \& Boyle, 2000).

Jodan and Purves (1993) asserted that writers usually inhabit cultural contexts in

literary texts. Literary works created by people from different backgrounds represent their own identities, and images and serve as memoranda for their descendants. According to Soter (1997), "the power of literature to transport readers into other worlds has never 
been doubted by those who, despite their own worlds, have been captured by writers no matter how different the culture they inhabit" (p. 214). Soter (1997) further pointed out that readers will play the role as insiders to understand the culture if they are familiar with the sociocultural and political context of the literary setting.

Further, different human groups have different understandings of culture (Fairchild, 1967; Tapp, 2007). Literature, as a part of culture, could be regarded as a mirror that reflects the accumulated culture. To be more specific, literature can serve ideally as a true reflection of what the society is, who humans are, and why the world has become as it is (Tapp, 2007). Spears-Bunton (1992) held the view that literature plays the role of facilitating individuals in decoding the mystery of their culture. By learning literature, one can be familiar with a certain culture. As Spears-Bunton (1992) pointed out, "literature provides us with a way of looking at how members use language to codify knowledge, determine relevance and make connections between past heroes and prophets, and present concerns and situations" (p.46).

In a sense, literary texts are culturally embedded; texts could be regarded as cultural documents that reflect all kinds of accumulated culture at racial, ethnic, national, regional, and local levels. In sum, literature, literacy, and culture are three forces constantly interacting with each other, shaping each other and ultimately affecting people now and those generations to come.

As different human groups have preconceived cultural perspective, reading the text is to read it in the light of the culture. To some extent, how to interpret the literary works as intimate parts of their culture becomes an essential issue. However, for the current teaching practice of culturally diverse literary texts, the crucial reality is that the 
importance of cultural understanding is often neglected or given minimal consideration in practical literature classes.

Dealing with such problems, a growing body of research suggests that the pedagogy used by teachers who are successful with students in teaching culturally diverse literary texts can be described as a culturally-responsive approach. Such research suggests that cultural awareness of multiple identities in different races, classes, and ethnicities needs to be integrated into school curricula (Dolby, 2000; Gay, 2000; Herbert, 2001). The interrelationship of culture, literature, and literacy should receive more attention in the teaching of literary works. Lin (1994) viewed the relationship between culture and literature as something like whole and part. As Lin (1994) stated, "a culture can exist and still be divided into literature, music, etc., but literature cannot exist without a culture to portray and illuminate, and to be influenced by" (p. 27).

\section{Pedagogy Issues and Suggestions in Teaching Literary Texts}

With regards to pedagogy issues in literature teaching, Grossman (2011) stated close reading is still used as the main instructional method to teach literature in current schools. Students are always required to answer the questions about the text raised by teacher, and they are expected to develop the skills of "close reading." Realizing that relatively little research had looked closely at the benefits of different instructional approaches for the teaching of literature, Grossman (2011) provided us with lines of research in the teaching of literature that have attempted to build instructional models. Overall, these models are based on theoretical analyses of how the readers make meaning

from fictive texts. According to Grossman (2011), Marshall's study (1987) looked at how different writing tasks shaped students' response to literature; Smith's (1989) study of 
different approaches to the teaching of irony in poetry devised an instructional unit that explicitly taught students to recognize five clues to ironic meaning. Smith (1989) compared the differences between the direct and tacit approaches for students in literature learning. According to the findings of the research, the least experienced readers benefited most from the direct approach while more experienced readers support the values of textual experiences and immersion. Carol Lee's (1993) study created a literature unit. It was built on the African-American discourse genre of signifying and helped students use their cultural knowledge of signifying to understand complex literary texts. Grossman (2011) also discussed about the differential effects of different approaches to the teaching of literature, incorporating alternative modes of response into the literature classrooms and the uses of alternative modes of response to literature.

Grossman (2001) further identified three main aspects in teaching literature: (a) small group discussions of literature; (b) discussion and student achievement; (c) literature learning at home. Grossman (2001) presented an overview of related lines of research in each subsection. Based on the research findings, corresponding suggestions for discussions of literature could be summarized as follows: (a) classroom discussions need to provide opportunities for transactions between readers and texts and foster specific ways of talking and thinking about literature; (b) research needs to pay attention to the roles of teachers and students in the small-group discussions of literature; (c) research needs to delve into the nature of activities or tasks that are assigned to small groups, and how these tasks embody the interpretive skills discussing literature and allow diverse students to contribute to the construction of meaning; (d) teachers who use group work must understand the literature on how groups can exacerbate exiting status 
difference among group members and must structure tasks to distribute the intellectual leadership of the groups more equitably; (f) research needs to explore instructional models that allow students to take on the primary work of constructing meaning and to illustrate how teacher scan help students grow in their interpretative powers; (e) research needs to help teachers shift from the teacher-dominated recitations to more open-ended discussions of literature (Grossman, 2001).

Besides reviewing the fundamental pedagogy and research in literature teaching from a holistic perspective, some scholars have addressed specific pedagogical approaches to teaching literature through cultural understanding for college-level students, especially in the aspect of constructing new knowledge through a critical cultural perspective.

Jordan and Purves (1993) explored the challenges confronted by both teachers and students, while they are reading texts that are from their own culture, or they are reading texts that are different from their own culture. It concerned their understanding of the specific texts of one or more of the target cultures (African American, Asian, Native American, Hispanic/Latino, and Anglo-European). The major challenges that students have while reading literature include:(a) incapable to read the texts within a cultural context; (b) rejected the text as alien because of stereotyping; (c) the misunderstanding of the texts because of readers' pleasant interpretations; (d) readers' personal judgment to texts from different perspectives (Jordan \& Purves, 1993). The study indicated that on the one hand, teachers have no exact idea "how they can best influence students to see the same cultural concerns they have: (Jordan \& Purves, 1993, p.19). On the other hand, "students have trouble reading texts from cultures other than their own, because they 
have little knowledge of other cultures and little practice in reading literature as the expression of a culture and an author who is influenced by the culture" (Jordan \& Purves, 1993, p.19).

Hines (1997) identified different approaches that four teachers used in literature classrooms: (a) a new critical perspective that incorporates the text-centered and teacherled orientations to literature instruction; (b) a reader-response orientation that encourages students to join a classroom community where reader knowledge and experience are valued dimensions of the reading experience; (c) a social justice framework that raises social justice issues and allows students to "read" culture; and (d) cultural criticism that challenges and critiques received "ways of seeing" in the literary texts. In presenting the four different teaching approaches, Hines (1997) held the view that "knowledge, language, and truth are socially constructed; thus students can assert, contest, and complicate truth claims in the classroom" (p. 118).

Scholars (Milner, 1983; Beach, 1997; Hines, 1997; Banks, 1996) have suggested that teachers need to consider as important how to deal with conflicts between personal and cultural knowledge and to employ cultural understanding of the literary works as a means of teaching literature. Milner (1983) argued that since personal and cultural knowledge is problematic when it conflicts with scientific ways of validating knowledge, it is oppositional to the culture of the school, or challenges the main tenets and assumptions of mainstream academic knowledge. Much of the knowledge about outgroups that students learn from their home and community cultures consists of misconceptions, stereotypes, and partial truths. Several scholars (Beach,1997; Hines, 1997; Soter, 1997) have pointed out that students have few opportunities to learn 
firsthand about the cultures of people from different racial, ethnic, cultural, religious, and social-class groups in literature classes. According to Banks (1996), "the concepts, explanations, and interpretations that students derive from personal experiences in their homes, families, and community cultures constitute personal and cultural knowledge. The assumptions, perspectives, and insights that students derive from experiences in their homes and community cultures are used as screens to view and interpret the knowledge and experiences that they encounter in the school and in other institutions within the larger society" (p.51). Banks (1996) claimed that an important goal of education in literature teaching is to free students from their cultural and ethnic boundaries and enable them to cross cultural borders freely, although the school should recognize, validate, and make effective uses of students' personal and cultural knowledge in instruction. Clearly, the challenge that teachers face is how to make effective instructional use of the personal and cultural knowledge of students while at the same time helping them to reach beyond their own cultural boundaries in teaching literature.

In Banks' (1996) opinion, literature teaching aims at helping students to understand how knowledge is constructed. From Banks's (1996) point of view, teachers in traditional literature classes, tend to transmit predetermined literature knowledge to the students. They pay a lot of attention to telling students what each piece of literature is about, dictate notes, create synopses, and character-studies and so on. They also ask students to do summaries of plots and themes, identify certain characteristics that represent its period of genre, certain traits of style and structure. Since such mechanical pedagogy shows little concern to the students' own appreciation toward the text, it fails to recognize students as the primary actors in constructing literary world. Banks (1996) 
have proposed two possible ways that teachers could implement this in literature class. One approach is to facilitate students' understanding regarding how the knowledge is constructed and how the knowledge reflects the social context. Another approach is to share students' own cultural experiences and interpretations of events (Banks, 1996). In these ways, students could have more opportunities to investigate and determine how cultural assumptions, frames of references, perspectives, and the biases within a discipline influence the ways that knowledge is constructed. They will also have more opportunities to create knowledge themselves and identify ways in which the knowledge they construct is influenced and limited by their personal assumptions, positions, and experiences.

Fickel (2000) has suggested that teachers could integrate political and cultural context into literature classes and encourage students to reflect the social reality critically by reading literature. Fickel (2000) pointed out that teaching students' basic reading and writing skills in literary works is the first step for teachers in class. The more important issue for teachers is to help students to acquire mental habits that will lead to literary insight, critical judgment, and ethical and social understanding. Fickel (2000) also pointed out that the sources of literature derive from social and cultural reality. When teachers explicitly engage students in interrogating the social, political and economic forces widely existing in literary works, students could come to understand the text as social, political, and ideological statements that reflect conceptions of right, good and truth (Fickle, 2000).

Beach (1997) have suggested that teachers could adopt an ethnographic approach to teach literary texts. An ethnographic approach means that students are provided with 
opportunities to observe and experience characters' lives in the field or have access to the first-hand resources that could reflect the authentic culture in the text (Beach, 1997). To be specific, teachers could require students to explore deeply particular cultural traditions or norms depicted in the text through interviewing people who are insiders of the culture, collecting written material or visual resources that are related to the cultural context of the literary works, and write reflections that record their progress in cultural understanding of the literary works. According to Flood (2002), students play the roles of cultural anthropologist by using an ethnographic approach. They seek understandings of the cultural patterns and practices of everyday life of the group under study from an emic or insiders' perspective (Flood, 2002); they explore the culture through constant reading and observing. They confirm and contrast what they listened to, what they saw and what they read, and eventually uncover the ways in which insiders view the world; how the insiders construct the patterns of life; and how they construct values, beliefs, ideas, and symbolic-meaningful systems. 


\section{CHAPTER III}

\section{METHODS}

This chapter describes methods for the study. It includes refined research questions, participants and settings, procedures, instruments, research design, data collection and measurements, as well as research hypotheses and statistical models. The ten research questions in the Research Questions section of this Chapter are the extensions of two main research questions in Chapter 1, as the groups using a cultural criticism approach were further divided into two experimental groups, which was determined by the proficiency levels that the same instructor implemented the cultural criticism approach for the first time and the second time. Participants and settings were described later followed by specific procedures for carrying out this research. Then the instruments and the two-fold research design are presented. Five types of collected data and how they are measured in the research are introduced in the section of Data Collection and measurements. After that, ten research hypotheses and related statistical models are demonstrated respectively. Finally, a summary of data analysis using seven statistical models are presented.

\section{Research Questions}

A central focus of my study was to investigate the relative effectiveness of using different pedagogical approaches in literature teaching and learning. The relative effectiveness was assessed for two essential aspects, namely, students' cultural understanding and literature comprehension. The researcher was also interested in understanding the impact of a cultural criticism approach, when an instructor was at different levels of proficiency of the instruction. So the student groups in the extended 
research questions below refer to three divided groups including two experimental groups and one control group. The partition of the two experimental groups depended on the proficiency levels of the same instruction using a cultural criticism approach for the first time and second time.

The concept of cultural understanding was further broken down into five components: (a) customs and beliefs (b) gender roles; (c) economic status; (d) politics; and (e) free will. The research question on students' cultural understanding of literary texts in Chapter 1 could be extended into the following subsidiary research questions:

1. Do students instructed by different literature teaching methods, on average, perform differently in the post-test of cultural analysis essay?

2. Do students instructed by different literature teaching methods, on average, perform differently in the components of post-test of cultural analysis essay?

3. Does the mean change in the general improvement scores between pre-test and post-test in cultural analysis essay differ among the student groups instructed by different literature teaching methods?

4. Does the mean change in the improvement scores on the components of cultural analysis essay between pre-test and post-test differ among the student groups instructed by different literature teaching methods?

Next, the concept of literature comprehension was further broken down into three subcategories: (a) identification of themes, purposes, plot development; (b) cultural and historical context; and (c) literary analysis.

For students' general performance on literature comprehension, the research question can be stated as follows: how different are students in three groups in their 
general performance on literature comprehension across three time points of testing? The above research question can be reflected in the following subsidiary research questions:

5. Does each group of students have different average performance scores in three British literature comprehension tests?

6. Do students in three groups, on average, have different performance scores for British literature comprehension test 1 , British literature comprehension test 2 , and British literature comprehension test 3 ?

Then the next research question is regarding students' performance on three subcategories of literature comprehension. In particular, how different are students in three groups in their performance on three subcategories of literature comprehension across three time points of testing? It can be reflected in the following subsidiary research questions:

7. Do students for each group, have different average performance scores on subcategories of literature comprehension in three tests?

8. Do students in three groups, on average, have different average performance scores on subcategories of literature comprehension for Test1, Test2, and Test3?

\section{Participants and Settings}

The inclusion criteria of participants in this study were as follows: (a) Chinese undergraduate English majors are at the intermediate/advanced level in English language acquisition competencies, such a characteristic enables them to read English literature; (b) these students are learning English literature in the regular class sessions in an undergraduate English program in China. 
In addition, G-Power was used to determine an adequate sample size, when the effect size is set as 0.15 , and the statistical power is set as $0.8, \alpha=0.05$. According to Gpower, the number of participants in this quantitative study should be 105 participants.

On the basis of the aforementioned criteria, 109 junior-year English majors who were enrolled into British literature course in Fall 2015 participated in this study. The duration of the course was from August 26 to December 16, 2015. Students were assigned into four classes. In the first two weeks, students in all classes were assigned with the same introduction of the course. For the rest of the semester, that is, from September 9 to December 9, 2015, Class 1 and Class 2 were instructed by a cultural criticism method while Class 3 and Class 4 were instructed by a traditional formalist and biographical method. The numbers of students in Class 1-4 were 27, 28, 26, and 28 respectively.

In the present study, students in the four classes formed three groups, which represent either the treatment or the control group that received different types of instruction. Here, Group 1 was the same as the Class 1, Group 2 was the same as the Class 2, and Group 3 included both Class 3 and 4. In other words, students who received traditional formalist and biographical instruction were placed into Group 3 as the control group; students under cultural criticism instruction were partitioned into two experimental groups. Table 1 shows a summary of the 3 groups of participants.

The partition was developed in order to assess the effect of different level of proficiency in teaching using a cultural criticism method. It allows to investigate the difference of the two experimental groups where the instructor presented different levels of familiarity with class procedure under the cultural criticism instruction at two separate 
class schedules. In fact, although both Class 1 and 2 are taught by the same instructor, Class 1 was always taught in an earlier time than Class 2. The time difference allowed the instructor to become more familiar with the teaching materials and application of the teaching method. The instructor could adjust the instructional method for teaching Class 2 according to feedback in Class 1.

Table 1

Three-group Partition of All Participants in This Study

Group Index Description

Group 126 students were in Group 1. They were instructed with the culture criticism method. The instructor was Zhang. She had no prior experience with the teaching method.

Group 28 students were in Group 2. They were instructed using the culture criticism method. The instructor was Zhang. She had more proficiency in using a cultural criticism method for Group 2 than Group 1.

Group 352 students were in Group 3. They were instructed using the traditional biographical and formalism instruction. The instructor was Li.

It is worth mentioning that the total number of students in Table 1 is 106 instead of 109. This is because there were three students whose data was either missing or incomplete after the completion of collecting and organizing data.

All of participants were from Department of English, School of Foreign Languages, in Anhui Agricultural University (AAU). Anhui Agricultural University is a public university in Hefei, Anhui Province, China. As a first-tier university, AAU offers a wide range of academic disciplines in agriculture, engineering, science and liberal arts at 
college level and graduate level. It has five postdoctoral research stations, 37 doctoral disciplines as well as 89 Master degrees across all disciplines. Among all programs, agriculture and engineering are the featured programs at AAU.

The School of Foreign Languages at AAU consists of five departments: Department of English, Department of Japanese, Department of French, Department of College English Education, and Department of English Education for Graduate Students \& Language Research Institute. There are roughly 400 undergraduate students in the English program. The English program is entirely located in the Department of English. After being enrolled into the program, students are assigned into four core classes, where they complete all mandatory courses. About $75 \%$ participants are from different cities and regions of Anhui Province. The rest of participants come from other provinces in mainland China. Most of the participants are 21 or 22 years old. By their junior year, students had learned a wide range of English courses (Appendix A), and completed the Test for English Majors, Band 4 (Appendix B). In other words, they have acquired extensive language acquisition ability through learning various English courses for English majors and completing Test for English Majors, Band 4 (TEM-4).

The study was implemented through a British literature course (Appendix C), which is mandatory for all the junior English majors at AAU. The course mainly includes an overview of British literary history and intensive readings of selected literary texts of the classical literary figures in different British literary periods. The objective of this course is to enhance students' understandings of Western culture and to develop their interpretative abilities in literature appreciation. The course is a 2-credit course. It takes a 
total of 32 semester hours, distributed throughout the entire 16-week regular semester at the pace of 2 hours per week.

\section{Procedures}

The study started in late August 2015 and was completed by late December 2015, the same schedule as the aforementioned British English Literature course at AAU. The following sections include curriculum design of the British literature course, information about the two course instructors, cultural criticism units of instruction, traditional units of instruction, and fidelity of implementation.

\section{Curriculum Design of British Literature}

The two instructors used the same textbook in the four literature classes. The mandatory textbook is Anthology of British Literature and History edited by Peilan Wang published in 1999 by Northeast Normal University Press, China. It mainly outlines the classical literary figures and provides their representative literary works in different British literary periods. The Anthology had several pieces of literary works for each British literary period, so the two instructors selected together one piece of specific literary work for each literary period from the textbook as the assigned reading materials for students. Prior to the beginning of the semester, the two instructors held a meeting to discuss the assigned readings and the pacing guide. There was a total of seven literature units. Each unit was completed within two weeks. The class sessions were two hours in a block schedule per week. The study started in the first week of school according to university calendar. In Week 1 and Week 2, students were first given an overview of the course as well as a lesson followed by a sample teaching model using either a cultural criticism method or a traditional formalist and biographical approach, then the class 
moved forward to discuss a specific literary work in a specified literary period per two weeks from Week 3 to Week 15. In Week 16 students were guided to review all the lessons.

Table 2 shows the reading schedule from Week 3 to Week 15 for all British literature classes.

Table 2

Reading Schedule for All British Literature Classes

\begin{tabular}{llll}
\hline Chapter & Literary Period & Selected Literary Texts & Week \\
\hline 1 & The Middle Ages & Beowulf & Weeks 3-4 \\
2 & The English Renaissance & Hamlet & Weeks5-6 \\
3 & $\begin{array}{l}\text { The period of Revolution and } \\
\text { Restoration }\end{array}$ & Paradise Lost & Weeks 7-8 \\
4 & The Age of Enlightenment & Gulliver's Travel & Weeks 9-10 \\
5 & The Romantic Period & Pride and Prejudice & Weeks 10-11 \\
6 & The Victorian Age & Oliver Twist & Weeks 12-13 \\
7 & Modernism & Mrs. Dalloway & Weeks 14-15 \\
\hline
\end{tabular}

\section{Instructors}

This study was conducted with two instructors at the same school site. Both instructors were female. Below is the description of each teacher for the British literature course.

Instructor A. Instructor A was the researcher of this study. She taught two experimental classes independently using cultural criticism. She was an adjunct faculty member in the Department of English at AAU. She holds a Master's Degree in Theory of Literature at Anhui University and an Educational Specialist Degree in Curriculum and 
Instruction with a focus in English Education from Florida International University. She had taught undergraduate courses both in China and the U.S for five years by 2015.

Instructor B. Instructor B taught another two control classes using traditional teaching methods (biographical criticism/formalism). She had been a college-level professor in British literature for seven years at AAU. She holds a Master's degree in English Literature and Language from Anhui Normal University, China.

Below was an overview of the two instructional steps in experimental/control classes.

\section{Cultural Criticism Units of Instruction}

In the classes that use cultural criticism approach, each class session consisted of students' presentations, discussions, the instructor's feedback and assignments. See Figure 1 below (developed by researcher) for the specific procedures using a cultural criticism approach:

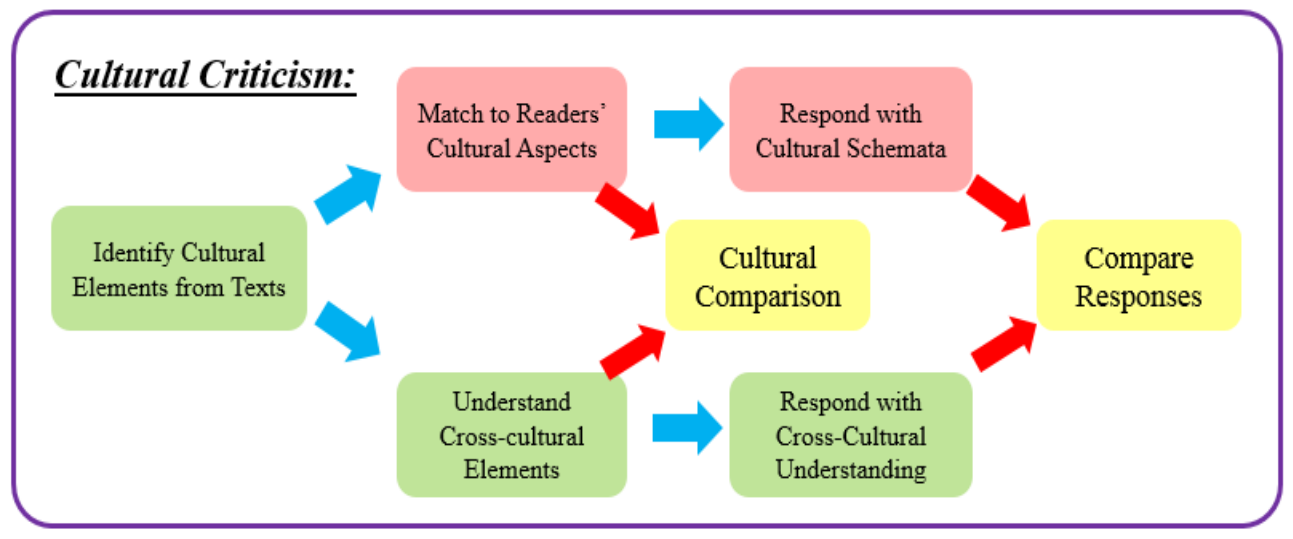

Figure 1. A flow chart for cultural criticism teaching procedure.

For further explanation, using cultural criticism in teaching literature can be described as the following steps: 
1. Instructor proposed the tentative discussion topics and themes from the assigned literary works in each literary unit.

2. Students identified cultural elements and concepts from the text and from their own cultural schemata separately.

3. Students responded to the text separately based on the two types of culture in step 2. Students compare their responses and provide a corrected response. Specifically, in step 1, the tentative discussion topics selected by the instructor should have reflected a certain aspect of culture at the time that the literary texts contextualize. For example, any topic that is related to the customs, politics, religion, economy and society etc. Following these criteria, the discussion topics proposed by the instructor include: tribal culture in Beowulf, humanism in Hamlet, religious perspectives in Paradise Lost, imperialism in Gulliver's Travel, marriage in Pride and Prejudice, poverty and criminality in Oliver Twist, feminism in Mrs. Dalloway for each literature unit.

In step 2, first, students are required to identify cultural elements within the assigned texts. These elements are also needed to be extended to more generic cultural concepts in the era of the text. The extension could be derived from related literature, documents, and online resources. In fact, the extended cultural concepts for British literature are an approximation of the authentic cultural context and social backgrounds reflected in the text. For example, in the discussion model of Romeo and Juliet (Appendix D), marriage is the proposed discussion topic. Under the general concept of marriage, several cultural elements related to specific context of the literary texts could be identified further, such as Romeo and Juliet's love for each other, aristocracy's 
marriage choices, family's authority on marriage, human rights towards marriage, betrayal of arranged marriage. Second, students are required to identify the counterpart cultural aspects (or domestic culture) from their own culture. These domestic cultural elements are instantiations of cultural schemata which is different from the cultural referents within the text.

In step 3, first, students respond to the text feature based on the domestic cultural concepts. Such a response is an instantiation of the impacts of cultural schemata from their own culture. Meanwhile, another response based on the identified cultural concepts should be made as well. The two responses are further to be compared so that the students can understand how cultural schemata yield a difference from students' cultural understandings of select literary texts and their comprehension of the cultural referents within the selected texts. Finally, a corrected response should be summarized, which mitigates the influences from students' cultural schemata.

In week 1, students were provided an overview of the course. They were also introduced to the concept of cultural criticism in literature teaching. In week 2, students were given a first guide for practicing cultural criticism in learning literature. In particular, a sample of text from the literature was selected and a discussion model (Appendix D) was provided. The students were also given five essential questions for class discussion. The questions were derived from the following considerations:

1. "What kinds of behavior, what models of practice, does this work enforce?

2. Are there differences between your values and the values implicit in the work you are reading?

3. Upon what social understandings does the work depend? 
4. What are the larger social structures with which these particular acts of praise or blame (that is, the text's apparent ethical orientation) might be connected?" (Greenblatt, 2007, p.226).

In the discussion model, marriage in Romeo and Juliet had been selected as discussion topic in the class. Students were provided with several pieces of assigned texts in the play of Romeo and Juliet. These assigned texts (Appendix D) reflect the cultural concepts related to the discussion topic of marriage, such as class consciousness towards marriage, an individual's right on the choice of marriage, and arranged marriage between two families. Based on the identified cultural concepts in the marriage, students explored the content of social cultural phenomenon in search of related documents/literature when the play of Romeo and Juliet was written. For example, student may explore the social context of arranged marriage between two families in Shakespeare's time. After that, students connected the related cultural concepts in marriage with their own culture, and compared the differences between the values towards marriage in their own culture and the values towards marriage implicit in the literary texts they were reading.

By the end of the week 2, students were divided into six study groups. Each group consisted of four or five students. They could self-select to join in any group they may have had interest in leading class discussions in the following weeks. Starting from week 3, each student group took turns to lead a class discussion for the assigned literary texts in each literary unit. The instructor provided discussion topics and their related cultural concepts for the leading group's reference. Two weeks before the formal class discussion, the instructor met the leading group and assisted the group in defining the cultural concepts that the students felt had emerged from the texts. The instructor checked if the 
cultural concepts defined by the leading group are closely related to her pre-selected discussion topic of the selected literary texts. Upon the instructor's approval for the defined cultural concepts, the group was required to complete the following tasks before the class:

1. Locate the related three pieces of literary texts that are related to the proposed discussion topic/themes and relevant cultural concepts, and then distribute them to the class one week ahead of the formal discussion;

2. Provide a rationale regarding how the discussion topic was reflected in the assigned literary texts;

3. Prepare five essential questions connecting with the social cultural influence on the preselected themes and the defined cultural concepts and distribute them to the class one week ahead of class time. Students were also provided a specific direction on raising five essential questions related to their selected literary texts: (a) students should work on why and how questions rather than factual questions; (b) the questions should be open-ended, interesting questions, rather than a fact question, that is, they concern about the characters' actions, decisions and choice; or they focus on explaining characters' actions/decision/choice and the underlying reasons influenced by the social culture; or they value diverse perspectives; (c) the questions should be relevant to the selected literary pieces; (d) the questions should be connected to the social culture influence.

4. Complete a critical cultural incident study (Yu, 2005). This assignment (Appendix D) requires that the group prepare a five-page narrative writing pieces that are related to the instructor-selected themes of the literary text. In particular, for the 
critical cultural incident study, the student groups are required to focus on the theme and the elements of culture related to the theme, and then depict the cultural influences on the discussion topic, and analyze the possible causes of cultural phenomena. They are required to support their writings by showing evidence from the original literature sources.

During the class, the responsibilities that the leading group has include:

5. Lead discussion about the assigned reading toward an examination of the social and cultural contexts emerged from the texts.

6. Present answers to the five essential questions that are used for guiding class discussion.

7. Present the cultural incident study to the class.

The instructor is responsible for the following:

1. Facilitate the discussion by ensuring the implementation of cultural criticism approach;

2. Help identify cultural schemata and cultural elements in the texts;

3. Comment whether students' responses appear to be based on their own cultural understanding.

Prior to the in-class discussion, all of students should complete the assigned readings and answer all the questions provided by the leading group.

In each class session, a Know-Wonder-How Learn (KWHL; Appendix E) were assigned to all of students. The KWHL chart helped learners organize the following learning aspects: what they know, what they want to know, how they will learn, and what they have learned in the class. For this class, students were required to first activate their 
prior knowledge about the defined cultural concepts. Then they continued to update the chart in the process of discovering the defined cultural concepts in class. After class, students examined what they have learned from the class in terms of using cultural criticism to appreciate literature. In addition, students were asked to complete one cultural-response assignment designed by the instructor per week (Appendix F) This assignment guided students to use a cultural criticism approach in the reading process. More specifically, students not only were required to identify the cultural elements that are unfamiliar to them, but to also used the cultural elements such as the personal, social, cultural context of the text to evaluate on its own cultural terms.

\section{Traditional Units of Instruction}

The traditional model primarily uses biographical criticism and formalist criticism to teach British literature. In the classes, the instructor mainly follows the major steps below for each class session:

1. provides an overview of a particular literature period;

2. presents the biography of the authors;

3. asks students summarizing the main ideas of literary works;

4. assists students in analyzing plot developments;

5. ensures students have a close reading of selected literary texts.

Overall, the way the students approached literature was to examine a text systematically. This included listing all the possible themes of a particular literary work, summarizing the plot developments, analyzing the main characters and events, and identifying the literary techniques as well as devices. Under this circumstance, the 
reading is only for a practical purpose - knowing the content of the text and the themes or the author's attitudes, etc.

The model for teaching Romeo and Juliet using traditional formalist and biographical method is also included in Appendix G. In this model, after giving students an overview of the Renaissance literary period and its social context, the instructor introduced students to the literary figure of William Shakespeare's biography, the characteristics of his literary works at different stages influenced by social factors. The main idea of Romeo and Juliet was also presented by the instructor. Then students were provided a piece of selected literary script for a close reading. Several questions were put forward in order to assist students in understanding the specific content in great detail for Act II, Scene II of the play. For example, scripts of Act II, Scene II were provide with students. It mainly described Romeo and Juliet's love speech in the Capulet's garden. The following question are put forward by the teacher: What does Romeo wish for, as he watches Juliet lean her cheek on her hand?

\section{Fidelity of Implementation}

A professor of English at AAU served as the observer for this study. Throughout the semester, he visited each of the four literature classes in the study one time randomly. Prior to the onset of the semester, the researcher provided a full-day training session to help the observer understand the two different literature instruction methods: cultural criticism and traditional formalist/biographical criticism. During the training, the researcher did the following: (a) introduced essential concepts of the pedagogical theory of two approaches; (b) shared related teaching practices generated from several published academic articles with the observer; (c) explained to the observer about the general 
course design, specific teaching procedures of each method; (d) showed the observer two teaching models using a cultural criticism approach and traditional formalist and biographical criticism method. Finally, the researcher provided him with copies of observer checklists. The observer was also informed not to share the information on the checklist with the other instructor. The checklist included the basic guidelines of teaching procedures that were adapted from cultural criticism units of instruction as well as traditional units of instruction respectively (Appendix $\mathrm{H}$ ). The researcher discussed how each item listed on the checklist could be implemented specifically in the previous provided two teaching models. In addition, the researcher answered the observer's questions about why the course design followed a cultural criticism approach, cultural criticism units of instruction, and the main difference of two literary teaching approaches, etc. The researcher also assessed the observer's knowledge by asking him questions about the two models of instruction. The observer not only presented a summary of two models of instruction, but also connected specific teaching procedures of those two methods with some specific examples in the literature teaching and learning. In this way, the observer was able to discern the pedagogical differences between the two teaching approaches. Every time the observer visited the class, he observed whether the instructor followed the step in each unit of instruction, and whether the instructor did not. He then put a check in each box as appropriate on the checklist. He could also add additional comments for the overall impression of the lessons. A total of four class observations were completed finally, two observations were for classes under the cultural criticism instruction; and another two observations were for classes under the traditional formalist and biographical instruction. The observer stayed through the entire class for each 
observation. Based on the results on the checklists, two classes as experimental groups employed a cultural criticism approach, and another two classes as control groups utilized traditional formalist and biographical approach.

Table 3

Summary of All Tests Assigned to Participants

\section{Test Notation Description}

CulTestA Cultural analysis pre-test; It contains five components: (a) customs and beliefs towards marriage (b) gender roles concerning marriage; (c) economic status concerning marriage; (d) politics concerning marriage; and (e) free will concerning marriage. The test was taken on Aug. 26Sept.2, 2015

CulTestB Cultural analysis post-test; It contains the same five components as CulTestA. The test was taken on Nov. 18-24, 2015

CompTest1 The $1^{\text {st }}$ test on British literature comprehension. It contains three components: (a) identification of themes, purposes, and plot development; (b) cultural and historical context; and (c) literary analysis. The test was taken on Oct. 14, 2015

CompTest2 The $2^{\text {nd }}$ test on British literature comprehension. It contains the same three components as CompTest1. The test was taken on Nov. 15, 2015

CompTest 3 The $3^{\text {rd }}$ test on British literature comprehension. It contains the same three components as CompTest1. The test was taken on Dec. 16, 2015

TEM4 Test for English Major Band 4. This is a nationwide standard English test for English major students. The test had been taken on April, 2015, before the study was conducted. 


\section{Instruments}

A total of five tests were administrated to students in all classes. They consisted of two essay tests (denoted as CulTestA and B) and three literature comprehension tests (denoted as CompTest1, 2, and 3). Table 3 shows a summary of all the tests and their notations.

On one hand, the CulTests evaluated students' performance on the cultural understanding within a particular literary work. They evaluated students' ability in capturing the cultural referents, contextualizing the social culture within the literary texts, and making connections between texts and the cultural contexts using cultural analysis. In particular, students were required to write an essay concerning the cultural analysis of Romeo and Juliet as a pre-test (Appendix I), and another essay concerning the cultural analysis of Pride and Prejudice as a post-test (Appendix J)

Both CulTests focused on one common theme of marriage and required students to develop an essay from the perspective of social cultural influences on the characters' choices of marriage. When students were depicting the cultural influences on the choice of marriage for the characters and analyzing the possible causes of the characters' choice/values in his/her marriage in the literary texts, they were prescribed to incorporate into the essay five components of cultural analysis: (a) customs and beliefs towards marriage (b) gender roles concerning marriage; (c) economic status concerning marriage; (d) politics concerning marriage; and (e) free will concerning marriage. To be specific, the researcher used a deductive coding method (Hsieh \& Shannon, 2005) to design these five components. In deductive coding, a coding scheme or categories could be derived from theories (Hsieh \& Shannon, 2005). The listed above five cultural components were 
derived from the general concept of culture. Culture refers to "a nation's civilization, psychological structure of the nation, spiritual pursuits, cultural customs, religion, history, economy, political system and other aspects of ideology form different facets" (Zhen, 2012, p.36). Based on the definition of culture above, the researcher developed a coding scheme to decompose the two pieces of literary works that have a common theme of marriage into five cultural components.

The format of these essay writing tests was modified from one assignment in a published online syllabus ENGL 640 Early American Literature and Culture developed by Gregory Eiselein in Spring 2007 at Kentucky State University. Upon checking various online sources, the researcher found that this assignment could help collect students' performance scores regarding cultural understanding of literary works. To be specific, this assignment built on the premise that understanding a particular culture will help us better appreciate and comprehend that culture's literary productions. The pre-test was administrated before the instruction started; and the post-test was administrated after the students received instruction for three months. Students were given one week to complete the test.

On the other hand, the CompTests evaluated students' performance from the perspective of literature comprehension. The tests were designed in a similar way to the Graduate Record Exam Literature in English Test (GRE-sub). They were categorized into three categories (the same as the GRE sub exam categories): (a) cultural and historical context; (b) identification of themes, purposes and plot development; and (c) literary analysis. Questions in the CompTests were selected from multiple test banks such as the College Level Examination Program (CLEP): analyzing and Interpreting literature, 
Test for English Majors, Band 4 (TEM), Graduate Student Entrance Examination for Chinese English Majors, as well as the British literature tutorial books. The questions included objective ones in the form of multiple choices and subjective ones in the form of literary discussion and analysis. The researcher first double-checked to make sure the selected test questions were placed under the relative category. For example, under the section of cultural and historical context, in CompTest1, question 3, 4, 5, 6 were designed for testing students' knowledge on the basic cultural and historical concepts of the Movement of Renaissance; in CompTest2, question 1, 2, and 5 were designed for testing students' knowledge on the basic cultural and historical concepts of the Movement of and Enlightenment. In CompTest3, question 1-10 were designed for testing students' knowledge on the features, trend, and main thoughts of different literary period in Britain, which also reflect the information regarding cultural and historical context or background. Under the section of identification of themes, purposes and plot development, the questions were selected based on the representative literary works that students have learned from each literary period, such as from Hamlet, Gulliver's Travels, and Pride and Prejudice, etc. The purpose of these questions was to examine students' understanding of the possible themes, the author's attitude and the essential ideas of plots towards a particular literary work. Under the section of literary analysis, in CompTest1, selections from Hamlet and Sonnet 18 from William Shakespeare as well as related comprehension and analysis questions were provided for students; in CompTest2, selections from Pride and Prejudice and one poem from William Wordsworth as well as related comprehension and analysis questions were provided for students; in CompTest3, reading comprehension multiple choice test questions were designed followed by one 
literary criticism on Hamlet, one poem from Percy Shelly, selections from Pride and Prejudice. There are another three literary analyses and interpreting questions focusing on Hamlet's internal conflict in the play of Hamlet and Satan's characteristics in Paradise Lost. In order to ensure that the questions from the test bank covered the three relevant aspects comprehension, the researcher also invited another two teachers in English literature at AAU to assess the content appropriateness of the three CompTests. The two teachers were given one week to have an overview of the content of each test. For each review, they highlighted the questions that they deemed inappropriate under each subcategory, provided brief comments behind the question, and returned the draft of test to the researcher. Based on two teachers' feedback, the researcher revised the question or deleted the question that did not match with the content area under each subcategory, and when necessary, the researcher also consulted with two teachers for some of unclear comments and discussed with them further on their content appropriateness as well as re-designing of the test questions. This procedure helped the researcher assess the content validity of the instrument. More specifics on the content of the questions for three CompTests could be found in Appendix L, Appendix M, and Appendix N.

The first test was administrated on October 14, 2015 when students learned specific literary works and related literary history in the Middle Ages, the English Renaissance, and Period of Revolution and Restoration. The second test was administrated on November 15, 2015 when students had learned literary works and related literary history in the Age of Enlightenment, and the Age of Romanticism. For the above two tests, students were given 45 minutes to complete them. The third test was 
administrated on December 16, 2016 when the students have learned all the content of course. Students were given 90 minutes to complete it.

\section{Research Design}

The study compared the effect of different methods on students' cultural understanding of literary works as well as on their literature comprehension in teaching a British literature course at an English program of a Chinese university.

On evaluating the effect of different teaching methods on students' cultural understanding of literary texts, the study used a quasi-experimental, pre-test, post-test design by analyzing students' scores in pre-test and post-test with respect to the general performance and related five components of cultural analysis essay. The study was conducted using a quasi-experimental design as the researcher used the pre-existing classes of junior English majors at an English program in a Chinese university, there was no random selection of students for this study.

On evaluating the effect of different teaching methods on students' literature comprehension, the study used repeated measure mixed design to track and analyze students' performance in a sequence of tests, both from the perspective of students' general performance and related three subcategories in three British literature comprehension tests. A repeated measure mixed design investigates not only the effect of test occasion/time (within-subject variation), but also the effect of the treatment (between-group variation). In this study, repeated measure mixed design included two levels, ANCOVA mixed design and MANCOVA mixed design. In a repeated measure ANCOVA mixed design, the partitioning of total variance is adjusted with the variance introduced by the covariate. In a repeated measure MANCOVA mixed design, the 
measured dependent variables are in multi-dimensional space. In this study, a repeated measure ANCOVA mixed design was used to determine whether the adjusted population mean of the scores on literature comprehension differ over three tests and/or among the three groups, and a repeated-measure mixed design MANCOVA was conducted to determine whether the adjusted population mean of the scores on the three subcategories of British literature comprehension differ over three tests and/or among the three groups.

\section{Data Collection and Measurement}

The data collection in this study kept participants anonymous by only using the last five digits of participants' student identification number instead of their names. Participants' records including their homework, exam and respective scores were kept private in the research. Only the researcher had access to the information collected in this project. They were kept in locked storage at the university as well as the researcher's computer for a period of time upon the completion of the research. As a result of the limited data collection, no other privacy related information was collected from each individual participant.

The following categories of data have been collected from participants during this study.

1. Students' performance scores in the two cultural analysis tests

2. Students' performance scores in the three literature comprehension tests.

3. Students' TEM 4 scores

Notations and descriptions of those scores are listed below. 


\section{Data Collected from Cultural Analysis Essay}

The data collected from each cultural analysis test first consisted of five components: (a) customs and beliefs towards marriage (b) gender roles concerning marriage; (c) economic status concerning marriage; (d) politics concerning marriage; and (e) free will concerning marriage. Each of the five components counted for $20 \%$ of the total score. Notation and descriptions are listed in Table 4.

Table 4

Summary of Components in a Culture Analysis Test

\section{Component Notation Description}

TestX_Custom This is the component of customs and beliefs towards marriage Account for $20 \%$ of total score in CulTestA and CulTestB (Remark: $\mathrm{X}=\mathrm{A}$ or $\mathrm{B}$. The same applies to the rest of the table)

TestX_Gndr

This is the component of gender roles concerning marriage Account for $20 \%$ of total score in CulTestX

TestX_Econ

This is the component of economic status concerning marriage Account for $20 \%$ of total score in CulTestX

TestX_Pol

This is the component of politics concerning marriage Account for $20 \%$ of total score in CulTestX

TestX_Free This is the component of free will concerning marriage Account for $20 \%$ of total score in CulTestX 
The difference of the score on the same component from the two culture analysis tests is called the improvement score for the component. For example, the value of (TestB_Free - TestA_Free) represents the students' improvement from CulTestA to CulTestB in the component of free will in marriage. The improvement scores of components are denoted as ImproveCustom, ImproveGndr, ImproveEcon, ImprovePol and ImproveFree. Those are used in Cultural Analysis Tests section of Chapter 4.

In order to assess students' ability for the cultural understanding of British literary texts in the CulTest, a scoring rubric had also been developed by the researcher with FIU professors in the area of English Education as well as English Language and Literature. The total scores of the CulTest are 100 points. Each category of cultural component accounts for 20 points. Among the five cultural components, each category consists of four evaluation criteria: (a) knowledge of British culture; (b) ability to identify cultural norms/values in British culture; (c) articulation of identified cultural norms/values in British culture; and (d) ability to connect students own cultural analysis to analysis of British cultural values in marriage. Each criterion has three levels with the scores ranging from 0 to five points at different levels (Appendix K). They were designed for evaluating the content quality of students' understanding of the particular cultural aspect in the British literary texts. The rationale for designing the four evaluation criteria was based on the evolving process of cultural knowledge learning. Criterion (a) assessed students' general British cultural knowledge on the conceptual level that could be learned from any source. Criterion (b) assesses students' ability in identifying particular cultural customs, beliefs, values or attitude that are representative of British cultures within the literary texts. At this point, students are capable of distinguishing the features of British culture 
from other cultures rather than simply staying at the conceptual level of learned cultural knowledge. Criterion (c) assesses students' comprehension of the aspects of narration, organization and analysis on the identified cultural norms/values of British culture in the flow of the literary text. Criterion (d) assesses students' ability in integrating their own cultural perspectives in analyzing and understanding a particular cultural aspect within the literary texts. They can make a comparison between their own culture and the British culture within the texts; they can also make a connection based on what they have known from their culture and what they have learned from the culture within the texts.

\section{Data Collected from British Literature Comprehension Test}

\section{Table 5}

Summary of Components in a Literature Comprehension Test

\section{Component Notation Description}

CCXN This is the component of cultural and historical contexts. It counts for $25 \%$ of the total score in CompTestX.

Here $\mathrm{X}$ represents the index of the test, i.e. CC1N, CC2N and $\mathrm{CC} 3 \mathrm{~N}$ are representing the score of this component in CompTest1, CompTest2, and CompTest3 respectively.

$N$ represents the score is normalized across the three test for the purpose of fair comparison. The same applies to the rest.

IDXN

This is the component of identification of themes, purpose, and plot developments. It counts for $25 \%$ of the total score in CompTestX.

LAXN This is the component of literary analysis. It counts for $50 \%$ of the total score in CompTestX. 
Data collected from each literature comprehension test consisted of three components: (a) identification of themes, purposes, and plot development; (b) cultural and historical context; and (c) literary analysis. Notation and description are listed in Table 5.

Measures of scores in literature comprehension were taken from the British Literature Comprehension Test (CompTest1, CompTest2 and CompTest3). British Literature Comprehension Tests covered the following categories: (a) cultural and historical context (denoted as CC); (b) the identification of themes, purpose and plot developments (denoted as ID); (c)literary analysis (denoted as LA). The total scores of the test are 100 points. For CompTest 1 and CompTest2, they contained 10 multiple choice questions in CC section, which accounted for 25 points out of 100 points; 10 multiple choice questions in ID section, which account for another 25 points out of 100 points. The remaining 50 points are distributed in the literature analysis section. This consists of objective literary analysis subsection and subjective literary analysis subsection. In the objective subsection, two pieces of reading on selected literary texts were provided first, and the multiple-choice questions that were designed for testing students' literature comprehension followed each piece of reading. They account for 40 points. One literary analysis question account for 10 points, which was designed to test students' ability in interpreting assigned literary texts such as plot development, character analysis or understandings of features of literary texts. For CompTest3, it contained 20 multiple choice questions for the $\mathrm{CC}$ section, which account for 15 points out of 100 points; 10 multiple choice questions for the ID section, which account for another 15 points out of 100 points. The remaining 70 points are distributed in the LA section. It 
consists of objective literary analysis subsection and subjective literary analysis subsection. In the objective subsection, three pieces of reading on selected literary texts were provided first, and the multiple choices questions that followed each piece of reading. They account for 30 points. Three subjective literary analysis questions account for another 40 points.

General test scores as well as scores on each of these components were returned as the data for the CompTest1, 2 and 3. In addition, since the score distribution for relative subcategories in different CompTest was different, so the scores were normalized to the same scale for fair comparison purpose.

\section{Collected TEM-4 Scores}

Students' TEM 4 scores were also collected within the semester. This was mainly used as covariate to analyze the data in British literature comprehension tests under some ANCOVA and MANCOVA model for repeated measure mixed design in Chapter 4. As TEM4 is a standardized English exam with an emphasis on students' comprehensive ability to use English as a foreign language (Wen, 2010), so it could reflect students' integrative English language competencies prior to the instruction. The students have taken TEM-4 on April ,2015, and the scores collected at the beginning of October, 2015.

\section{Summarized List of Collected Data}

After the completion of collecting and organizing data, it was found there were three students whose data was either missing or incomplete among a total of 109 students who were enrolled into the British literature course. To be specific, 1 student in Class 1 missed 4 tests except for attending CompTest3; 1 student in Class 3 missed CulTestA; 
and 1 student in Class 4 was absent in CompTest2. Finally, the researcher collected and coded the data from 106 participants as raw data.

This included:

- All component scores TestA_Custom, TestA_Gndr, TestA_Econ, TestA_Pol and TestA_Free for CulTestA (Notations defined in Table 4)

- All component scores TestB_Custom, TestB_Gndr, TestB_Econ, TestB_Pol and TestB_Free for CulTestB

- All component scores CCIN, IDIN and LAIN for CompTest1 (Notations defined in Table 5)

- All component scores CC2N, ID2N and LA2N for CompTest2

- All component scores CC3N, ID3N and LA3N for CompTest3

- All TEM4 scores (Notations defined in Table 3)

The following intermediate data were derived from the raw data

- The total scores for CulTestA and CulTestB as the sum of their component scores respectively.

- The total improvement score Improve from CulTestA to CulTestB

- All improvement scores of components in cultural analysis tests: ImproveCustom, ImproveGndr, ImproveEcon, ImprovePol and ImproveFree (as defined earlier in the Section of Data Collected from Cultural Analysis)

All data above are inputs to SPSS operations that are detailed in the section of Cultural Analysis Tests and section of Literature Comprehension Tests in Chapter 4 for assessing students' cultural understanding and literature comprehension. 


\section{Inter-Rater Reliability}

Four raters (Teachers Zhang, Shu, Wang and Li) participated in the grading

process. To be specific, four raters, Zhang, Shu, Wang and Li, graded the tests on cultural understanding of literary texts; two raters, Zhang and Li graded the tests on literature comprehension. The researcher Zhang was the primary rater in the study, she graded all the tests on cultural understanding of literature texts as well as literature comprehension; rater Wang is an associate professor of English at Anhui Agricultural University; rater Li is a senior lecturer of English at Anhui Agricultural University; rater Shu is a lecturer of English at Anhui Sanlian College. For CulTests, the researcher provided all the raters with the scoring rubric and further explained to them the specific criteria to assess students' cultural understandings of the relevant literary works in CulTests. The researcher first completed grading students' essays in one class and selected three students' sample essays by herself that represented different levels in cultural understanding of literary work (excellent, good, pass, failure respectively) to the raters. These student sample essays were assessed by the researcher's self-developed scoring rubric. Once the student sample essays were selected by researcher, they were also assigned for the other raters for the initial grading trial. A half-day training session for assessing CulTests were arranged by the researcher prior to the other raters' grading CulTests formally. In the training session, the researcher demonstrated to the raters how she used the scoring rubric in assessing students' different levels in cultural understanding of literature work. She connected the evaluation criteria with specific examples from graded students' essays for further explanations. After that, all the raters were invited to share their results and perspectives in grading the students' sample essays. 
For the controversial issues arising in the grading process, the researcher discussed with the raters further for clarification. She also answered the other raters' questions and concerns. In this way, the raters were sufficiently trained in using the rubric to assess the CulTests before starting grading. All the raters were not able to see the students' names as well as the class number while they were grading the essays. Rater Zhang graded CulTestA and CulTestB for all four classes; rater Shu graded CulTestA and CulTestB for two classes; and rater Li graded CulTestA and CulTestB for the other two classes. Rater Wang graded the returned essays from the researcher that pended re-evaluation. To be specific, once three raters (Rater Zhang, Rater Zhang Shu and Rater Li) completed grading in CulTest, the researcher compared the grading scores from two raters who graded the same two classes, the allowable error in each cultural component of the essay was within 5 points. If the error was beyond this range, the researcher returned it to rater Wang for re-evaluation. The final scores for each cultural component depended on two independent raters' average grading scores, and the difference of these two raters' respective grading scores in each cultural component should be within minimum difference. For example, for a particular student's essay in a certain class, it was graded by both rater Zhang and Rater Shu. It was found that the difference of the scores between these two raters in at least a cultural component of the essay were beyond 5 points, so rater Wang joined in the re-evaluation per the researcher's requirement. After Wang completed grading, the researcher compared three independent raters' scores in each cultural component and found the minimum scoring difference between any pairwise raters, either Zhang and Shu or Wang and Zhang. Then these two raters' grading scores in a certain cultural component were used as valid scores for grading, and the mean of 
them were calculated as the final scores of each cultural component. Finally, the final scores of each cultural component were accumulated into the total scores. For the CompTests, the researcher also gave the raters the key points and evaluation criterion in subjective questions in CompTests. Rater Zhang graded all the objective sections; the remaining subjective sections were distributed to two raters, Zhang and Li respectively. They took turns to evaluate all the subjective questions on literary analysis for all the four classes for this section, and then the final scores for the subjective answers were the average scores of the two raters.

\section{Research Hypotheses and Statistical Models}

Using the research questions put forward in the Research Questions section of Chapter 3, the hypotheses were further developed after the researcher established the measures.

The research hypotheses on students' cultural understanding of literary texts were as follows:

Research Hypothesis 1a: Student groups taught by a cultural criticism approach achieve higher performance mean scores in the post-test CulTestB than student groups taught by a traditional formalist and biographical approach, after controlling the covariate scores of pre-test CulTestA.

In order to test Research Hypothesis 1a, Simple Group Main Effects Test for particular values of covariate was conducted to evaluate whether the post-test means differ significantly among the groups using different literature methods after adjustment of the covariate scores. Independent variable is the group factor, included three levels, that have been defined in Table 1, in the Participants and Settings section of Chapter 3. 
The dependent variable was the students' performance scores in post-test CulTestB. Pretest scores CulTestA served as covariate.

Research Hypothesis 1b: Student groups taught by a cultural criticism approach achieve higher performance mean scores than student groups taught by a traditional formalist and biographical approach in the post-test CulTestB .

In order to test Research Hypothesis 1b, one-way ANOVA was conducted to evaluate whether the post-test means differ significantly among the groups using different literature methods. Independent variable is the group factor, included three levels, that have been defined in Table 1, in the Participants and Settings section of Chapter 3. The dependent variable was the students' performance scores in post-test CulTestB.

Research Hypothesis 2: Student groups taught by a cultural criticism approach achieve higher performance scores in the aspects of customs and beliefs, economics status, gender, politics and free will than student groups taught by a traditional formalist and biographical approach for the post-test CulTestB, after controlling the covariate scores of pre-test CulTestA.

In order to test Research Hypothesis 2, one-way MANCOVA were conducted to determine whether the post-test means for the students' performance scores on the five components differ among the groups using different literature methods, with adjustment of pre-test scores. Independent variable is the group factor, included three levels, that have been defined in Table 1, in the Participants and Settings section of Chapter 3. The dependent variable was the students' performance scores on the five components in posttest CulTestB. Pre-test scores CulTestA served as covariate. 
Research Hypothesis 3: Student groups taught by a cultural criticism approach achieve higher improvement scores than student groups taught by a traditional formalist and biographical approach between pre-test and post-test of cultural analysis essay.

In order to test Research Hypothesis 3, one-way ANOVA was conducted to evaluate whether the general improvement scores differ significantly among the groups using different literature methods. The independent variable, the group factor within three levels that have been defined in Table 1, in the Participants and Settings section of Chapter 3. The dependent variable was the students' improvement scores between the pre-test and post-test.

Research Hypothesis 4: Student groups taught by a cultural criticism approach achieve higher improvement scores in the aspects of customs and belief, economics status, gender, politics and free will between pre-test and post-test of cultural analysis essay than student groups taught by a traditional formalist and biographical approach.

In order to test Research Hypothesis 4, one-way MANOVA was conducted to evaluate whether the improvement scores in the respective five cultural components differ significantly among the groups using different literature methods. The independent variable, the group factor with three levels that have been defined in Table 1, in the Participants and Settings section of Chapter 3. The dependent variable was the students' improvement scores in the five components between the two CulTests.

The research hypotheses on students' literature comprehension were as follows:

Research Hypothesis 5: Student groups taught by a cultural criticism approach achieve higher performance scores in the average of three British literature 
comprehension tests than student groups taught by a traditional formalist and biographical approach, after controlling the covariate TEM4 scores.

Research Hypothesis 6: Student groups taught by a cultural criticism approach have higher performance scores in British literature comprehension test 1, British literature comprehension test 2, and British literature comprehension test 3 respectively than student groups taught by a traditional formalist and biographical approach, after controlling covariate TEM4 scores.

In order to test Research Hypothesis 5 and Research Hypothesis 6, a repeatedmeasure mixed design ANCOVA was conducted to determine whether the adjusted population mean of the scores on literature comprehension differ over three tests and/or among the three groups. The within-subjects factor was time with three levels that have been defined in the Participants and Settings section of Chapter 3. The between-subjects factors were group with three levels that have been defined in Table 1, in the Participants and Settings section of Chapter 3. The dependent variables were students' performance scores in each test. The covariate was the TEM4 scores.

Research Hypothesis 7: Student groups taught by a cultural criticism approach achieve higher performance scores for the aspects of cultural and historical contexts, identification of themes, purpose, and plot developments, as well as literary analysis in three British literature comprehension tests than student groups taught by a traditional formalist and biographical approach, after adjustment of covariate TEM4 scores.

Research Hypothesis 8: Student groups taught by a cultural criticism approach achieve higher performance scores in the aspects of cultural and historical contexts, identification of themes, purpose, and plot developments, as well as literary analysis in 
CompTest1, CompTest2, and CompTest 3 respectively than student groups taught by a traditional formalist and biographical approach, after controlling the covariate TEM4 scores.

In order to test Research Hypothesis 7 and Research Hypothesis 8, a repeatedmeasure mixed design MANCOVA was conducted to determine whether the adjusted population mean of the scores on the three subcategories of British literature comprehension differ over three tests and/or among the three groups. The within-subjects factor was time with three levels that have been defined in the Participants and Settings section of Chapter 3. The between-subjects factors were group with three levels that have been defined in Table 1, in the Participants and Settings section of Chapter 3. The dependent variables were students' performance scores in three subcategories of each test. The covariate was the TEM4 scores.

\section{Data Analysis}

Multiple statistical procedures were applied to the data analysis. They included Simple Group Main Effects Test, one-way Analysis of Variance (ANOVA), one-way Analysis of Covariance (ANCOVA), one-way Multivariate Analysis of Variance (MANOVA), one-way Multivariate Analysis of Covariance (MANCOVA), Repeated Measure Mixed-design Analysis of Covariance (RM-ANCOVA) and Repeated Measure Mixed-design Multivariate Analysis of Covariance (RM-MANCOVA). SPSS were used to analyze the data. Preliminary introductory information regarding the aforementioned statistical models could be found in Appendix O. Detailed results are presented in Chapter 4. 
On the effect of different teaching methods on students' cultural understanding of literary texts, the study used a quasi-experimental, pre-test, post-test design with the purpose of understanding whether the post-test means differ among the groups. The research first compared students' post-test mean scores on the relative six dependent variables (students' general performance scores and five cultural components scores) in the CulTests among three groups. Simple Group Main Effects Test, one-way analysis of variance (ANOVA), as well as one-way multivariate of covariance (MANCOVA) were used. To be specific, one-way ANOVA was conducted to evaluate whether the post-test means differ significantly among the groups using different literature teaching methods. Simple Group Main Effects Test for particular values of covariate and one-way MANCOVA were conducted respectively to determine whether the post-test means for the students' general performance scores as well as five components scores differ among the groups using different literature teaching methods, with adjustment of pre-test scores. The research then compared students' mean scores of improvement between pre-test and post-test on the relative six dependent variables (students' general improvement performance score and improvement scores in the five cultural components) among three groups. To be specific, one-way ANOVA and one-way MANOVA were conducted respectively to evaluate whether the general improvement scores as well as improvement scores in the respective five cultural components differ significantly among the groups using different literature teaching methods.

On the effect of different teaching methods on students' literature comprehension, the study used repeated-measures mixed design with the purpose of understanding whether the mean change in the outcome over time differs among the groups. A repeated- 
measure mixed design ANCOVA, a repeated-measure mixed design MANCOVA were applied. A repeated-measure mixed design ANCOVA was conducted to determine whether the adjusted population mean of the scores on literature comprehension differ over three tests and/or among the three groups, a repeated-measure mixed design MANCOVA was conducted to determine whether the adjusted population mean of the scores on the three subcategories of British literature comprehension tests differ over three tests and/or among the three groups. 


\section{CHAPTER IV}

\section{RESULTS}

This study was undertaken to understand the difference that a pedagogy of cultural criticism might make on students' cultural understandings of selected English (British) literary texts and on their literature comprehension abilities. This chapter presents the descriptive analysis of all collected test scores, the tests of hypothesis concerning the relationship between different literature teaching instruction and student performance scores in cultural understanding of literature works and their literature comprehension, and the results of using multiple statistical analysis based on the research design in the Research Design section of Chapter 3. The results of data analysis were classified into two main categories: (a) the impact of instructional methods on cultural understanding of literature works and (b) the impact of instructional methods on literature comprehension.

\section{Descriptive Analysis of All Collected Test Scores}

This section presents a descriptive analysis of all collected test scores during Fall 2015 when the research was conducted at the site. It includes five general scores collected from all participants, scores of CulTestA, CulTestB, and improvement scores between CulTestA and CulTestB for students' cultural understanding of literary work, scores of CompTest1, CompTest2, and CompTest3 on students' literature comprehension, as well as TEM4 scores that assess students' levels of English language proficiencies. The purpose of presenting these scores in this section is to help understand 
students' general performance in the tests of cultural understanding of literary works, the tests of literature comprehension and a standardized comprehensive exam (TEM4) for assessing students' English language proficiencies at an English program of AAU.

CulTestA is the pre-test of students on their cultural understanding of literature works. The pre-test scores, CulTestA, served as a covariate for analyzing students' performance scores in cultural understanding of literature works. The general improvement scores were also obtained by subtracting from CulTestB by CulTestA. More details regarding the mean and standard deviation of CulTestA can be found in Table 26 (See Appendix P).

For the mean of CulTestA, group 2 had the highest mean $(M=57.66)$, group 1 had a lower mean $(M=54.08)$, and group 3 had the lowest mean $(M=40.25)$. More details regarding the mean and standard deviation of CulTestA can be found in Table 26 (See Appendix P).

CulTestB is the post-test of students on their cultural understanding of literature works. Group 2 had the highest mean $(M=78.27)$, group 1 had a lower mean $(M=68.31)$, and group 3 had the lowest mean $(M=40.07)$. More details regarding the mean and standard deviation of CulTestB can be found in Table 27 (See Appendix P).

The students' average improvement scores are derived from the mean difference between CulTestA and CulTestB. For the means of improvement scores, group 2 had the highest mean $(M=20.61)$, group 1 had a lower mean $(M=14.23)$, and group 3 had the lowest mean $(M=-.18)$. More details regarding the mean and standard deviation of CulTest Improvement Scores could be found in Table 28 (See Appendix P). 
CompTests include three tests on students' literature comprehension. In CompTest1, the mean of group $1(M=71.02)$ and group $2(M=71.34)$ were almost same, group 2 had a lower mean $(M=54.08)$, and group 3 had the lowest mean $(M=61.84)$. In CompTest2, group 2 had the highest mean $(M=66.38)$, group 1 had a lower mean ( $M=64.54)$, and group 3 had the lowest mean $(M=56.78)$. In CompTest3, group 2 had the highest mean $(M=74.79)$, group 1 had a lower mean $(M=71.96)$, and group 3 had the lowest mean $(M=69.84)$. More details can be found in Table 29 (See Appendix P).

TEM4 is a nationwide standard English test for English major students that examine students' integrative English language proficiency. More details can be found in Table 30 (See Appendix P). For the mean and standard deviation of TEM4, the three groups' scores are very close. The mean score of group 3 is 66.63 , the mean score of group 1 is 65.08 and the mean score of group 2 is 65.71 . More details can be found in Table 30 (See Appendix P).

\section{Culture Analysis Tests}

This section compares the experimental impacts of instruction of cultural criticism on students' cultural understanding of literary works. Systematic comparisons were performed among the performance of the three groups of students in cultural analysis tests. This includes three post-test oriented models: Simple Group Main Effects Test, one-way Analysis of Variance (ANOVA) and one-way Multivariate Analysis of Covariance (MANCOVA), as well as two improvement score oriented models: one-way Analysis of Variance (ANOVA) and one-way Multivariate Analysis of Variance (MANOVA). A general description of those models can be found in the Appendix O. Results of CulTestA and CulTestB (defined in the Instrument section of Chapter 3) as 
well as the five component scores for each of cultural analysis test were stored into SPSS program to derive the results in this section.

\section{Simple Group Main Effects for Particular Values of the Covariate on the Post-test CulTestB}

The effect of different literature teaching methods on students' cultural understanding of literary works was first evaluated by comparing their performance on the post-test CulTestB. The students' performance in the pre-test may impact differently on the post-test and therefore the pre-test CulTestA scores were added as covariate in the comparison. The following research question was attempted to be answered:

Research Question: Do students instructed by different literature methods, on average, achieve different performance scores in the post-test CulTestB, assuming no prior differences in the students' performance scores in the pre-test CulTestA?

Null Hypothesis: There is no statistically significant difference for students in each group instructed by the different literature teaching methods on performance scores in the post-test CulTestB, after controlling for the covariate scores of pre-test CulTestA.

Alternative Hypothesis: There is a statistically significant difference for students in each group instructed by different literature teaching methods on performance scores in the post-test CulTestB, after controlling for the covariate scores of pre-test CulTestA.

Using $\mu_{i}$ to denote the population mean of scores in CulTestB for students in group $i$, the hypotheses can be represented as

$$
\begin{aligned}
& \mathrm{H}_{0}: \mu^{\prime}{ }_{1 \text { CulTest } \mathrm{B}}=\mu^{\prime}{ }_{2 \text { CulTest } \mathrm{B}}=\mu^{\prime}{ }_{3 \mathrm{CulT} \text { Test } \mathrm{B}} \\
& \mathrm{H}_{\mathrm{a}}: \mu^{\prime}{ }_{i \text { CulTest } \mathrm{B}} \neq \mu^{\prime}{ }_{k \text { CulTestB }} \text { for some } i, k
\end{aligned}
$$


A one-way analysis of covariance (ANCOVA) was planned to evaluate the effect of different literature teaching methods on the students' performance scores in post-test CulTestB, after control of the covariate CulTestA scores. An alpha level of .05 was set as the significant level. The independent variable, group factor, included three levels, that have been defined in Table 1, in the Participants and Settings section of Chapter 3. The dependent variable was the students' performance scores in post-test CulTestB. The covariate was the pre-test CulTestA scores.

Table 6

Selected Results of the Test of Homogeneity of Slopes

\begin{tabular}{lllllll}
\hline Source & $\begin{array}{l}\text { Type III Sum } \\
\text { of Squares }\end{array}$ & Mean Square & F & Sig. & $\begin{array}{l}\text { Partial Eta } \\
\text { Squared }\end{array}$ \\
\hline $\begin{array}{l}\text { Group } \\
\text { CulTestA }\end{array}$ & 1476.015 & 2 & 738.008 & 4.886 & .009 & .089 \\
Error & 15103.885 & 100 & 151.039 & & & \\
\hline
\end{tabular}

Note. group * CulTestA represents the interaction effect.

${ }^{\mathrm{a}} \mathrm{R}$ Squared $=.697($ Adjusted R Squared $=.682)$

A preliminary analysis was conducted to evaluate homogeneity of slopes between the covariate and the dependent variable across groups, an assumption underlying ANCOVA (Green \& Salkind, 2007). The interaction effect was significant, $F(2,100)=$ 4.866, $p=.009<.05$, so the slopes were heterogeneous in the population, ANCOVA is inappropriate to be further conducted. The partial $\eta^{2}$ for the interaction was .089 , indicating that the mean differences in the post-test CultTest B scores among three group instructed by different literature teaching methods varied moderately as a function of the 
pre-test CulTestA scores. Table 6 shows the interaction effect in the test of homogeneity of slopes.

Based on the significant results for the interaction effect, simple main effects tests were conducted that allow for heterogeneity of slopes instead of ANCOVA assuming the homogeneity of slopes. The research question would have to be modified since the slopes were found to be heterogeneous among groups.

Simple main effect tests were conducted to assess differences among groups at low (1 SD below the mean), medium (mean), and high (1 SD above the mean) values on the covariate. For this case, the mean and standard deviation on pre-test CulTestA scores, ignoring groups, are 48.24 and 17.76 respectively. Accordingly, low, medium and high values on CulTestA are 30.48, 48.24, and 66.00. A $p$ values of $.017(.05 / 3)$ was required for significance for each of these three tests. If any one simple main effect was significant, pairwise comparisons were evaluated at the same level (i.e., .017) as the simple main effects tests, following the LSD procedure.

The research question has to be restated as follows:

Do students in each group instructed by different literature methods, on average, achieve different performance scores in the post-test CulTestB, if the students' performance scores in the pre-test CulTestA are 30.48, 48.24, and 66.00 respectively?

Null Hypothesis: There is no statistically significant difference for students in each group instructed by different literature teaching methods on performance scores in the post-test CulTestB, if the students' performance scores in the pre-test CulTestA are $30.48,48.24$, and 66.00 respectively. 
Alternative Hypothesis: There is a statistically significant difference for students in each group instructed by different literature teaching methods on performance scores in the post-test CulTestB, if the students' performance scores in the pre-test CulTestA are $30.48,48.24$, and 66.00 respectively.

The statistical representation of the hypotheses remains the same as equation (1) and (2).

For individuals with a pre-test CulTestA score of 30.48, the adjusted means for the post-test CulTestB scores were estimated to be 55.72 for the group 1, 66.15 for the group 2, 40.11 for the group 3, respectively. More details regarding the mean, standard deviation as well as the $95 \%$ confidence intervals for the mean among the three groups after adjustment of covariate CulTestA scores, can be found in Table 31 (See Appendix P).

The simple main effects test was significant, $F(2,100)=18.669, p=.00$, partial $\eta^{2}=.272$. So the null hypothesis in Equation (1) should be rejected. The strength of relationship between different literature teaching methods and the students' performance scores on CulTestB, as assessed by $\eta^{2}$, was strong, with the different literature teaching methods accounting for $27.2 \%$ of the variance of the dependent variable. The $95 \%$ confidence intervals for the pairwise mean differences, and standard deviations for mean differences of the three groups after adjustment of covariate CulTestA, were reported in Table 7. From Table 7, it was found that group 1 and group 2 yielded significantly higher performance scores in post-test CulTestB than group 3, while the differences between group 1 and group 2 were not significant. 
For individuals with a pre-test CulTestA score of 48.24, the adjusted means for the post-test CulTestB scores were estimated to be 65.19 for the group 1, 74.07 for the group 2, 40.03 for the group 3, respectively. More details regarding the mean, standard deviation as well as the $95 \%$ confidence intervals for the mean among the three groups after adjustment of covariate CulTestA scores, are reported in Table 32.

Table 7

Pairwise Differences in Mean Changes for CulTestB Conditioned at Low CulTestA

\begin{tabular}{llllll}
\hline $\begin{array}{l}\text { Group (I) } \\
\text { Group (J) }\end{array}$ & Difference (I-J) & Std. Error & Sig. $^{\text {b }}$ & \multicolumn{2}{l}{$\begin{array}{l}\text { 95\% Confidence Interval for } \\
\text { Difference }^{\mathrm{b}}\end{array}$} \\
\cline { 3 - 6 } & & & .082 & -22.212 & 1.366 \\
\hline$(1)-(2)$ & -10.423 & 5.942 & .002 & 6.023 & 25.205 \\
$(1)-(3)$ & $15.614^{*}$ & 4.834 & $<.001$ & 17.015 & 35.059 \\
$(2)-(3)$ & $26.037^{*}$ & 4.547 & & & \\
\hline
\end{tabular}

The simple main effects test was significant, $F(2,100)=63.541, p<.001$, partial $\eta^{2}=.56$. So the null hypothesis in Equation (1) could be rejected. The strength of relationship between different literature teaching methods and the students' performance scores on CulTestB, as assessed by $\eta^{2}$, was strong, with the different literature teaching methods accounting for $56 \%$ of the variance of the dependent variable. The $95 \%$ confidence intervals for the pairwise mean differences, and standard deviations for mean differences of the three groups after adjustment of covariate CulTestA, are reported in Table 8, it was found that group 1 and group 2 yielded significantly higher performance scores in post-test CulTestB than group 3, while group 2 also yielded significantly higher performance scores in post-test CulTestB than group 1. 
For individuals with a pre-test CulTestA score of 66.00, the adjusted means for the post-test CulTestB scores were estimated to be 74.67 for the group 1, 81.99 for the group 2, 39.96 for the group 3, respectively. More details regarding the mean, standard deviation as well as the $95 \%$ confidence intervals for the mean among the three groups after adjustment of covariate CulTestA scores, are reported in Table 33.

Table 8

Pairwise Differences in Mean Changes for CulTestB Conditioned at Medium CulTestA

\begin{tabular}{|c|c|c|c|c|c|}
\hline \multirow[t]{2}{*}{$\begin{array}{l}\text { Group }(\mathrm{I})- \\
\operatorname{Group}(\mathrm{J})\end{array}$} & \multirow[t]{2}{*}{$\begin{array}{l}\text { Mean } \\
\text { Difference (I-J) }\end{array}$} & \multirow[t]{2}{*}{ Std. Error } & \multirow[t]{2}{*}{ Sig. $^{b}$} & \multicolumn{2}{|c|}{$\begin{array}{l}\text { 95\% Confidence Interval for } \\
\text { Difference }^{b}\end{array}$} \\
\hline & & & & Lower Bound & Upper Bound \\
\hline$\overline{(1)-(2)}$ & $-8.872^{*}$ & 3.650 & .017 & -16.113 & -1.630 \\
\hline$(1)-(3)$ & $25.161^{*}$ & 3.240 & $<.001$ & 18.732 & 31.590 \\
\hline$(2)-(3)$ & $34.032^{*}$ & 3.254 & $<.001$ & 27.576 & 40.489 \\
\hline
\end{tabular}

The simple main effects test was significant, $F(2,100)=46.866, p<.001$, partial $\eta^{2}=.484$. So the null hypothesis in Equation (1) should be rejected. The strength of relationship between different literature teaching methods and the students' performance scores on CulTestB, as assessed by $\eta^{2}$, was strong, with the different literature teaching methods accounting for $48.4 \%$ of the variance of the dependent variable.

The $95 \%$ confidence intervals for the pairwise mean differences, and standard deviations for mean differences of the three groups after adjustment of covariate CulTestA, were reported in Table 9. From Table 9, it was found that group 1 and group 2 yielded significantly higher performance scores in post-test CulTestB than group 3, while the differences between group 1 and group 2 were not significant. 
Table 9

Pairwise Differences in Mean Changes for CulTestB Conditioned at High CulTestA Group (I) - Mean Difference Std. Error $\quad$ Sig. $^{b} \quad$ 95\% Confidence Interval for Group (J) (I-J) Difference ${ }^{\mathrm{b}}$

\begin{tabular}{llllll} 
& & & \multicolumn{2}{c}{ Lower Bound Upper Bound } \\
\hline$(1)-(2)$ & -7.320 & 3.950 & .067 & -15.156 & .516 \\
$(1)-(3)$ & $34.708^{*}$ & 4.718 & $<.001$ & 25.348 & 44.068 \\
$(2)-(3)$ & $42.028^{*}$ & 4.416 & $<.001$ & 50.789 & 33.267 \\
\hline
\end{tabular}

Summary of findings. The data analytic results above have shown a significant difference among the performance of the three groups of students in terms of their posttest scores of cultural analysis. In particular, the two groups instructed by a cultural criticism approach obtained much better overall cultural understanding of literary work than the group of students who only received traditional formalist /biographical instruction. The results were obtained after controlling the potential impact of students' pre-test scores. Therefore, the null hypothesis in Equation (1) was rejected.

\section{One-way ANOVA for the Post-test CulTestB}

The different literature teaching methods on students' cultural understanding of literary works could also be evaluated by simply comparing their performance on the post-test CulTestB. The following research question was attempted to be answered:

Do students in each group instructed by different literature methods, on average, achieve different performance scores in the post-test CulTestB? 
Null Hypothesis: There is no statistically significant difference for students in each group instructed by different literature teaching methods on performance scores in the post-test CulTestB.

Alternative Hypothesis: There is a statistically significant difference for students in each group instructed by different literature teaching methods on performance scores in the post-test CulTestB.

Using $\mu_{i}$ to denote the population mean of scores in CulTestB for students in group $i$, the hypotheses can be represented as

$$
\begin{gathered}
\mathrm{H}_{0}: \mu_{1 \text { CulTest } \mathrm{B}}=\mu_{2} \text { CulTestB }=\mu_{3 \text { CulTest } \mathrm{B}} \\
\mathrm{H}_{\mathrm{a}}: \mu_{i} \text { CulTestB } \neq \mu_{k} \text { CulTestB } \text { for some } i, k
\end{gathered}
$$

A one-way analysis of variance (ANOVA) was conducted to evaluate the relationship between different literature teaching methods and the students' performance scores in post-test CulTestB. An alpha level of .05 was set as the significant level. The independent variable was the group factor with three levels as defined in Table 1, in the Participants and Settings section of Chapter 3. The dependent variable was the students' performance scores in post-test CulTestB. The ANOVA was significant, $F(2$, 103) $=83.97, p<.001$, so the null hypothesis in Equation (3) should be rejected.

The strength of relationship between the different literature teaching methods and the students' performance scores in post-test CulTestB, as assessed by partial $\eta^{2}$, was strong, with the different literature methods accounting for $62 \%$ of the variance of the dependent variable. Table 10 shows a summary of results of a one-way analysis of variance for CulTestB. 
Table 10

Summary of Results of a One-way Analysis of Variance for CulTestB

\begin{tabular}{lllllll}
\hline Source & $\begin{array}{l}\text { Type III Sum df } \\
\text { of Squares }\end{array}$ & Mean Square F & Sig. & $\begin{array}{l}\text { Partial Eta } \\
\text { Squared }\end{array}$ \\
\hline $\begin{array}{l}\text { Corrected } \\
\text { Model }\end{array}$ & $30897.942^{\mathrm{a}}$ & 2 & 15448.971 & 83.965 & $<.001$ & .620 \\
Group & 30897.942 & 2 & 15448.971 & 83.965 & $<.001$ & .620 \\
Error & 18951.294 & 103 & 183.993 & & & \\
Total & 395270.000 & 106 & & & & \\
Corrected Total & 49849.236 & 105 & & & & \\
\hline
\end{tabular}

Note. ${ }^{\text {a }}$ R Squared $=.620$ (Adjusted R Squared $=.612$ )

Follow-up tests were conducted to evaluate pairwise differences among the means. Table 11 shows the results of post hoc comparisons in Mean Changes for CulTestB. From Table 11, it was found that group 1 and group 2 yielded significantly higher performance scores in post-test CulTestB than group 3, while group 2 also yielded significantly higher performance scores in post-test CulTestB than group 1.

Table 11

The Results of Post hoc Comparisons in Mean Changes for CulTestB

\begin{tabular}{llllll}
\hline $\begin{array}{l}\text { Group (I) } \\
\text { Group (J) }\end{array}$ & $\begin{array}{l}\text { Mean } \\
\text { Difference } \\
(\mathrm{I}-\mathrm{J})\end{array}$ & Std. Error & Sig. & \multicolumn{2}{l}{\begin{tabular}{l} 
95\% Confidence Interval \\
\cline { 3 - 6 }
\end{tabular}} \\
\cline { 2 - 6 } & & & $\begin{array}{l}\text { Lower } \\
\text { Bound }\end{array}$ & Upper Bound \\
$(1)-(2)$ & $-9.9602 *$ & 3.54379 & .022 & -18.7711 & -1.1492 \\
$(1)-(3)$ & $28.2404 *$ & 3.38353 & $<.001$ & 19.9005 & 36.5803 \\
$(2)-(3)$ & $38.2005^{*}$ & 2.97458 & $<.001$ & 30.9104 & 45.4907 \\
\hline
\end{tabular}


Summary of findings. The data analytic results above have shown a significant difference among the performance of the three groups of students in terms of their scores of cultural analysis post-test. In particular, the two groups instructed by a cultural criticism approach obtained much better overall cultural understanding of literary work than the group of students who only received traditional formalist and biographical teaching. At the same time, for the two groups instructed by a cultural criticism approach, group 2 obtained much better overall cultural understanding of literary work than group 1. Therefore, the null hypothesis in Equation (3) is rejected.

\section{One-way MANCOVA for the Post-test CulTestB}

The different literature teaching methods on students' cultural understanding of literary works were then evaluated by comparing their performance on five aspects of the post-test CulTestB. The students' performance in the pre-test may impact differently on the post-test and therefore the pre-test CulTestA scores were added as covariate in the comparison. The following research question was attempted to be answered:

Do students in each group instructed by different literature methods, on average, achieve different performance scores in the aspects of customs and beliefs, economics status, gender, politics and free will for the post-test CulTestB, assuming no prior differences in the students' performance scores in the pre-test CulTestA?

Null Hypothesis: There is no statistically significant difference for students in each group instructed by different literature teaching methods on performance scores in the aspects of customs and beliefs, economics status, gender, politics and free will for the post-test CulTestB, after controlling for the covariate scores of pre-test CulTestA. 
Alternative Hypothesis: There is a statistically significant difference for students in each group instructed by different literature teaching methods on performance scores in the aspects of customs and beliefs, economics status, gender, politics and free will for the post-test CulTestB, after controlling for the covariate scores of pre-test CulTestA.

Using $\mu^{\prime}{ }_{i, \mathrm{C} \& \mathrm{~B},} \mu^{\prime}{ }_{i, \mathrm{Eco}}, \mu^{\prime}{ }_{i, \text { gender, }} \mu^{\prime}{ }_{i, \text { politics }}$ and $\mu^{\prime}{ }_{i, \mathrm{fw}}$ to denote the adjusted population means of scores for students in group $i$ at the five components in CulTestB, the hypotheses can be represented as,

$$
\mathrm{H}_{0}:\left\{\begin{array}{c}
\mu_{1 \mathrm{C} \& \mathrm{~B}}^{\prime}=\mu_{2 \mathrm{C} \& \mathrm{~B}}^{\prime}=\mu_{3 \mathrm{C} \& \mathrm{~B}}^{\prime} \\
\mu_{1 \mathrm{Eco}}^{\prime}=\mu_{2 \mathrm{Eco}}^{\prime}=\mu_{3 \mathrm{Eco}}^{\prime} \\
\mu_{1 \text { gender }}^{\prime}=\mu_{2 \text { gender }}^{\prime}=\mu_{3 \text { gender }}^{\prime} \\
\mu_{1 \text { politics }}^{\prime}=\mu_{2 \text { politics }}^{\prime}=\mu_{3 \text { politics }}^{\prime} \\
\mu_{1 \mathrm{fw}}^{\prime}=\mu_{2 \mathrm{fw}}^{\prime}=\mu_{3 \mathrm{fw}}^{\prime}
\end{array}\right.
$$

$$
\mathrm{H}_{\mathrm{a}}: \mu^{\prime}{ }_{i \mathrm{c}} \neq \mu^{\prime}{ }_{k \mathrm{c}} \text { for some group index } i, k \text { and some component } \mathrm{c}
$$

A one-way multivariate analysis of covariance (MANCOVA) was conducted to evaluate the effect of the three types of study strategies, on the five dependent variables, the scores in the aspects of customs and beliefs, economics status, gender, politics and free will for students in the three study groups. An alpha level of .05 was set as the significant level. The independent variable, group factor with three levels that have been defined in Table 1, in the Participants and Settings section of Chapter 3. The dependent variables were the corresponding five component scores in CulTestB. The covariate was the pre-test scores as CulTestA.

As the test for homogeneity of dispersion matrices is significant, $F(30$, 19517.57) $=3.237, p<.05$, the assumption of homogeneity of variance-covariance is violated, the Pillai's Trace Test was used to interpret the data in the further step. 
Significant differences were found among three groups instructed by different literature teaching methods. The Pillas's Trace Test is a test statistic that is very robust and not highly linked to assumptions about the normality of the distribution of the data (Caulcutt, 1991). In the multivariate tests, the Pilla's Trace $\vee=.567, F(10,198)=7.831, p<0.05$, so the null hypothesis in Equation (5) should be rejected. The multivariate $\eta^{2}$ based on Pilla's Trace was .283. The relationship between the covariate pre-test CulTestA scores and the dependent variables among three groups was significant, $F(5,98)=4.234, p<.05$, with the covariate accounting for about $17.8 \%$ (i.e. the partial $\eta^{2}$ of .178 of variance of the post-test CulTestB scores, controlling for the treatment factor of different literature teaching methods. More details regarding the means and the standard deviations on the five dependent variables for the three groups are reported in Table 34 (See Appendix P). Analysis of variance (ANCOVA) on the dependent variables were conducted as follow-up tests to the MANCOVA. The ANCOVA results for the scores on the aspects of customs and beliefs, economics status, politics, gender, and free will were significant.

For post-test scores in customs and belief, $F(2,102)=35.684, p<.05$, partial $\eta^{2}=.412$; For post-test scores in economics status $(2,102)=19.079, p<.05$, partial $\eta^{2}=.272$; For post-test scores in gender, $F(2,102)=16.755, \mathrm{p}<.05$, partial $\eta^{2}=.247$; For post-test scores in politics, $F(2,102)=52.591, p<.05$, partial $\eta^{2}=.508$; For post-test scores in free will, $F(2,102)=34.349, p<.05$, partial $\eta^{2}=.402$.

Table 12 contains a summary of results of a one-way analysis of covariance for five cultural components in CulTestB. 
Table 12

Summary of Results of a One-way Multivariate Analysis of Covariance for Five Cultural Components in CulTestB

\begin{tabular}{|c|c|c|c|c|c|c|c|}
\hline Source & $\begin{array}{l}\text { Dependent } \\
\text { Variable }\end{array}$ & $\begin{array}{l}\text { Type III } \\
\text { Sum of } \\
\text { Squares }\end{array}$ & $\overline{d f}$ & $\begin{array}{l}\text { Mean } \\
\text { Square }\end{array}$ & $\bar{F}$ & Sig. & $\begin{array}{l}\text { Partial Eta } \\
\text { Squared }\end{array}$ \\
\hline \multirow[t]{5}{*}{$\overline{\text { CulTestA }}$} & TestB_Custom & 71.942 & 1 & 71.942 & 9.822 & .002 & .088 \\
\hline & TestB_Econ & 140.141 & 1 & 140.141 & 11.632 & .001 & .102 \\
\hline & TestB_Gndr & 152.858 & 1 & 152.858 & 12.215 & .001 & .107 \\
\hline & TestB_Pol & 62.023 & 1 & 62.023 & 4.288 & .041 & .040 \\
\hline & TestB_Free & 66.226 & 1 & 66.226 & 4.564 & .035 & .043 \\
\hline \multirow[t]{5}{*}{ Group } & TestB_Custom & 522.714 & 2 & 261.357 & 35.684 & .000 & .412 \\
\hline & TestB_Econ & 459.731 & 2 & 229.866 & 19.079 & .000 & .272 \\
\hline & TestB_Gndr & 419.350 & 2 & 209.675 & 16.755 & .000 & .247 \\
\hline & TestB_Pol & 1521.343 & 2 & 760.672 & 52.591 & .000 & .508 \\
\hline & TestB_Free & 996.821 & 2 & 498.411 & 34.349 & .000 & .402 \\
\hline \multirow[t]{5}{*}{ Error } & TestB_Custom & 747.079 & 102 & 7.324 & & & \\
\hline & TestB_Econ & 1228.898 & 102 & 12.048 & & & \\
\hline & TestB_Gndr & 1276.457 & 102 & 12.514 & & & \\
\hline & TestB_Pol & 1475.329 & 102 & 14.464 & & & \\
\hline & TestB_Free & 1480.021 & 102 & 14.510 & & & \\
\hline \multirow[t]{5}{*}{ Corrected Total } & TestB_Custom & 1709.285 & 105 & & & & \\
\hline & TestB_Econ & 2264.443 & 105 & & & & \\
\hline & TestB_Gndr & 2278.389 & 105 & & & & \\
\hline & TestB_Pol & 3799.210 & 105 & & & & \\
\hline & TestB_Free & 3100.625 & 105 & & & & \\
\hline
\end{tabular}

The strength of relationship between factor on different literature teaching methods and dependent variables were very strong, as assessed by a partial $\eta^{2}$ with factor on literature teaching method accounting for $41 \%$ of the variance of the dependent 
variable on post-test scores in customs and belief, $27.2 \%$ of the variance of the dependent variable on post-test scores in economics status, $24.7 \%$ of the variance of the dependent variable on post-test scores in gender, $27.2 \%$ of the variance of the dependent variable on post-test scores in economics status, $50.8 \%$ of the variance of the dependent variable on post-test scores in politics, $40.2 \%$ of the variance of the dependent variable on post-test scores in free will, while controlling for the influence of pre-test CulTestA scores. The means of the post-test scores in each subcategory controlling the influence of the covariate pre-test CulTestA scores were ordered as expected across the three groups.

For the adjusted post-test mean scores in customs and beliefs, economics status, gender, politics, and free will, the same pattern happened across the five cultural components, that is, group 2 had the largest adjusted mean, group 1 had a smaller adjusted mean, and group 3 had the smallest adjusted mean. More details regarding the mean, standard deviation as well as the $95 \%$ confidence intervals for the mean among the three groups after adjustment of covariate CulTestA scores can be found in

Table 35 (See Appendix P). Follow-up tests were conducted to evaluate pairwise differences among these adjusted means for each cultural component. The same pattern happened across the cultural components in economic status, gender and free will. That is, based on the LSD procedure, the adjusted means for both group 1 and group 2 differed significantly from the group 3, but the adjusted means for group 1 and group 2 did not differ significantly. The same pattern happened across the cultural components in customs \& beliefs, and politics. That is, the adjusted means for both group 1 and group 2 differed significantly from the group 3, and the adjusted means for group 1 and group 2 also differ significantly. Table 13 contains the $95 \%$ confidence intervals for the pairwise 
differences, as well as the mean difference and standard deviations for mean differences of the three groups after adjustment of covariate CulTestA.

Table 13

Pairwise Differences in Mean Changes for Five Cultural Components in CulTestB

\begin{tabular}{|c|c|c|c|c|c|}
\hline \multirow[t]{2}{*}{$\begin{array}{l}\text { Dependent } \\
\text { Variable }\end{array}$} & \multirow[t]{2}{*}{$\begin{array}{l}\text { Group }(\mathrm{I})- \\
\operatorname{Group}(\mathrm{J})\end{array}$} & \multirow[t]{2}{*}{$\begin{array}{l}\text { Mean } \\
\text { Difference (I-J) }\end{array}$} & \multirow[t]{2}{*}{$\begin{array}{l}\text { Std. } \\
\text { Error }\end{array}$} & \multicolumn{2}{|c|}{$\begin{array}{l}\text { Sig. }{ }^{\text {b } 95 \% ~ C o n f i d e n c e ~ I n t e r v a l ~} \\
\text { for Difference }\end{array}$} \\
\hline & & & & \multicolumn{2}{|c|}{ Lower Bound Upper Bound } \\
\hline \multirow[t]{3}{*}{$\overline{\text { TestB_Custom }}$} & $(1)-(2)$ & $-1.569^{*}$ & .739 & $.036-3.035$ & -.102 \\
\hline & (1)-(3) & $4.029^{*}$ & 690 & .0002 .661 & 5.397 \\
\hline & $(2)-(3)$ & $5.597^{*}$ & .697 & .0004 .214 & 6.981 \\
\hline \multirow[t]{3}{*}{ TestB_Econ } & $(1)-(2)$ & -1.548 & .948 & $.106-3.429$ & .333 \\
\hline & $(1)-(3)$ & $3.724^{*}$ & .884 & .0001 .970 & 5.478 \\
\hline & $(2)-(3)$ & $5.272^{*}$ & .894 & .0003 .498 & 7.047 \\
\hline \multirow[t]{3}{*}{ TestB_Gndr } & (1)-(2) & -.855 & .967 & $.378-2.773$ & 1.062 \\
\hline & $(1)-(3)$ & $3.968^{*}$ & .901 & .0002 .180 & 5.756 \\
\hline & $(2)-(3)$ & $4.823^{*}$ & .912 & .0003 .015 & 6.631 \\
\hline \multirow[t]{3}{*}{ TestB_Pol } & $(1)-(2)$ & $-2.994^{*}$ & 1.039 & $.005-5.055$ & -.933 \\
\hline & $(1)-(3)$ & $6.648^{*}$ & .969 & .0004 .726 & 8.570 \\
\hline & $(2)-(3)$ & $9.642^{*}$ & .980 & .0007 .698 & 11.586 \\
\hline \multirow[t]{3}{*}{ TestB_Free } & $(1)-(2)$ & -1.921 & 1.041 & $.068-3.985$ & .144 \\
\hline & $(1)-(3)$ & $5.731^{*}$ & .971 & .0003 .806 & 7.656 \\
\hline & $(2)-(3)$ & $7.652^{*}$ & .982 & .0005 .705 & 9.599 \\
\hline
\end{tabular}

Summary of findings. The data analytic results above have shown a significant difference among the performance of the three groups of students in terms of their scores of cultural analysis post-test on the corresponding five aspects. In particular, the two groups instructed by a cultural criticism approach obtained much better cultural 
understanding of literary work on the five aspects than the group of students who only received traditional formalist and biographical teaching. For the two groups instructed by a cultural criticism method, group 2 taught by a cultural criticism approach second obtained much better cultural understanding of literary work on the aspect of custom \& beliefs, and politics than group 1 taught by a cultural criticism approach first. The results were obtained after controlling the potential impact of students' pre-test scores. Therefore, the null hypothesis in Equation (5) is rejected.

\section{One-way ANOVA for General Improvement scores between Two CulTests}

The different literature teaching methods on students' cultural understanding of literary works was then evaluated by comparing the general improvement they have made between the pre-test CulTestA and post-test CulTestB. The following research question was attempted to answered:

Does the mean change in the improvement scores between pre-test and post-test in cultural analysis essay differ among the three groups instructed by different literature methods?

Null Hypothesis: There is no statistically significant difference for students among three groups instructed by different literature teaching methods on improvement scores between pre-test and post-test of cultural analysis essay.

Alternative Hypothesis: There is a statistically significant difference among students in three groups instructed by different literature teaching methods on improvement scores between pre-test and post-test of cultural analysis essay.

Using $\mu_{i}$ to denote the population mean of the improvement scores from CulTestA to CulTestB for students in group $i$, the hypotheses can be represented as: 


$$
\begin{aligned}
& \mathrm{H}_{0}: \mu_{1 \text { imprv }}=\mu_{2 \text { imprv }}=\mu_{3 \text { imprv }} \\
& \mathrm{H}_{\mathrm{a}}: \mu_{i \text { imprv }} \neq \mu_{k \text { imprv }} \text { for some } i, k
\end{aligned}
$$

A one-way analysis of variance was conducted to evaluate the relationship between different teaching methods and the students' improvement scores between the pre-test and post-test. The independent variable was the group factor within three levels that have been defined in Table 1, in the Participants and Settings section of Chapter 3. The dependent variable was the students' improvement scores between the pre-test and post-test. The alpha was set at .05 significance level.

The ANOVA was significant, $F(2,103)=15.448, p<.05$, so the null hypothesis in Equation (7) should be rejected. Partial $\eta^{2}=.231$, indicated that different teaching methods accounted for $23.1 \%$ of the variance of the dependent variable. Table 14 provides a summary of results of a one-way analysis of variance for general improvement scores in CulTests.

Table 14

Summary of Results of a One-way Analysis of Variance for General Improvement Scores

\begin{tabular}{|c|c|c|c|c|c|c|}
\hline Source & $\begin{array}{l}\text { Type III Sum } \\
\text { of Squares }\end{array}$ & $\overline{d f}$ & Mean Square & $F$ & Sig. & $\begin{array}{l}\text { Partial Eta } \\
\text { Squared }\end{array}$ \\
\hline Corrected Model & $8865.873^{\mathrm{a}}$ & 2 & 4432.937 & 15.448 & $<.001$ & .231 \\
\hline group & 8865.873 & 2 & 4432.937 & 15.448 & $<.001$ & .231 \\
\hline Error & 29555.808 & 103 & 286.950 & & & \\
\hline Total & 46713.250 & 106 & & & & \\
\hline Corrected Total & 38421.682 & 105 & & & & \\
\hline
\end{tabular}
in CulTests.

Note. ${ }^{\mathrm{a}} \mathrm{R}$ Squared $=.231$ (Adjusted R Squared $=.216$ ) 
More details regarding the mean, standard deviation as well as the $95 \%$ confidence intervals for the general improvement scores between CulTestA and CulTestB among the three groups can be found in Table 36 (See Appendix P).

Follow-up tests were conducted to evaluate pairwise differences among the means. Because the homogeneity assumption was rejected in Levene's Test, $F(2$, $103)=3.407, p=.307<.05$, post hoc comparisons were conducted with the use of the Dunnett's C test, a test does not assume equal variances among the three groups.

There was a significant difference in the means between the group 1 and group 3, as well as group 2 and group 3; but there were no significant differences between the group 1 and group 2 . Table 15 reports the $95 \%$ confidence intervals for the pairwise differences, as well as the mean difference and standard deviations for mean differences on general improvement scores of the three groups.

Table 15

Pairwise Differences in Mean Changes for General Improvement Scores in CulTests

\begin{tabular}{llllll}
\hline $\begin{array}{l}\text { Group (I) } \\
\text { - Group } \\
\text { (J) }\end{array}$ & $\begin{array}{l}\text { Mean } \\
\text { Difference }\end{array}$ & Std. Error & Sig. & \multicolumn{2}{l}{$95 \%$ Confidence Interval } \\
\cline { 5 - 5 } & & & $\begin{array}{l}\text { Lower } \\
\text { Bound }\end{array}$ & Upper Bound \\
\hline$(1)-(2)$ & -6.3764 & 3.65571 & -15.4620 & 2.7093 \\
$(1)-(3)$ & $14.4135^{*}$ & 3.81269 & 5.0701 & 23.7568 \\
$(2)-(3)$ & $20.7898^{*}$ & 3.74849 & 11.6286 & 29.9510 \\
\hline
\end{tabular}

Summary of findings. The data analytic results above have shown a significant difference among the performance of the three groups of students in terms of their general improvement scores of cultural analysis essay between the pre-test and the posttest. In particular, the two groups instructed by a cultural criticism approach obtained 
much better cultural understanding of literary work than the group of students who only received traditional formalist and biographical teaching. Therefore, the null hypothesis in Equation (7) is rejected.

\section{One-way MANOVA for Improvement scores between Two CulTests}

The different literature teaching methods on students' cultural understanding of literary works was then evaluated by comparing the improvements they made in the corresponding five aspects between the pre-test and the post-test. The goal was to answer the following research question:

Does the mean change in the improvement scores on the aspects of customs and belief, economics status, gender, politics and free will between pre-test and post-test in cultural analysis essay differ among the three groups instructed by different literature methods?

Null Hypothesis: There is no statistically significant difference for students in three groups instructed by different literature teaching methods on improvement scores in the aspects of customs and belief, economics status, gender, politics and free will between pre-test and post-test of cultural analysis essay.

Alternative Hypothesis: There is a statistically significant difference for students in the three groups instructed by different literature teaching methods on improvement scores in the aspects of customs and belief, economics status, gender, politics and free will between pre-test and post-test of cultural analysis essay.

Using $\mu_{i, \mathrm{C} \& \mathrm{~B}}, \mu_{i, \mathrm{Eco}}, \mu_{i, \mathrm{gender}}, \mu_{i, \text { politics }}$ and $\mu_{i, \mathrm{fw}}$ to denote the population means of improvement scores from CulTestA to CulTestB for students in group $i$ at the five 
components, i.e. customs and belief, economics status, gender, politics and free will, the hypotheses can be represented as:

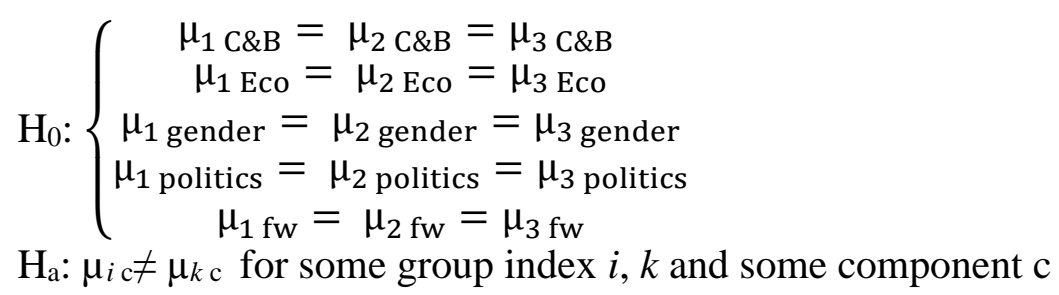

A one-way multivariate analysis of variance (MANOVA) was conducted to evaluate the relationship between different teaching methods and the students' improvement scores in each component between the pre-test and the post-test. The independent variable was the group factor with three levels that have been defined in Table 1, in the Participants and Settings section of Chapter 3. The dependent variable was the students' improvement scores in the five components between the two CulTests. The alpha was set at .05 significance level. The test for homogeneity of dispersion matrices was significant, $F(30,19517.57)=2.83, p<.05$, so Pillas's Trace would be used to interpret the data in the next multivariate tests. Significant differences were found among the three groups on the dependent measures. Pilla's Trace $\vee$ was of $.449, F(10,200)=5.79, p<.01$, so the null hypothesis in Equation (9) should be rejected. The multivariate $\eta^{2}$ based on Pilla's Trace was .224, indicating that different literature methods accounted for $22.4 \%$ of the variance of the dependent variable. Details regarding the means and the standard deviations on the dependent variables can be found in Table 37 (See Appendix P).

Analysis of variance (ANOVA) on the dependent variables were conducted as follow-up tests to the MANOVA. Each ANOVA was tested at the .05 level. The ANOVA on the improvement scores in custom and belief, politics and free will were significant. Improvement scores in customs and belief had the following data: $F(2$, 
$103)=9.715, p<.05$, partial $\eta^{2}=.159 ;$ improvement scores in politics had the following data: $F(2,103)=30.994, p<.05$, partial $\eta^{2}=.376$; improvement scores in free will had the following data, $F(2,103)=12.562, p<.05$, partial $\eta^{2}=.196$.

Table 16

Summary of Results of a One-way Multivariate Analysis of Variance for General Improvement Scores in Five Cultural Components of CulTests

\begin{tabular}{|c|c|c|c|c|c|c|c|}
\hline Source & $\begin{array}{l}\text { Dependent } \\
\text { Variable }\end{array}$ & $\begin{array}{l}\text { Type III } \\
\text { Sum of } \\
\text { Squares }\end{array}$ & $\overline{d f}$ & $\begin{array}{l}\text { Mean } \\
\text { Square }\end{array}$ & $\mathrm{F}$ & Sig. & $\begin{array}{l}\text { Partial Eta } \\
\text { Squared }\end{array}$ \\
\hline \multirow[t]{5}{*}{ group } & ImproveCustom & 349.239 & 2 & 174.620 & 9.715 & .000 & .159 \\
\hline & ImprvoeEco & 98.396 & 2 & 49.198 & 1.946 & .148 & .036 \\
\hline & ImproveGndr & 14.096 & 2 & 7.048 & .238 & .789 & .005 \\
\hline & ImprovePol & 1767.320 & 2 & 883.660 & 30.994 & .000 & .376 \\
\hline & ImproveFree & 730.870 & 2 & 365.435 & 12.562 & .000 & .196 \\
\hline \multirow[t]{5}{*}{ Error } & ImproveCustom & 1851.301 & 103 & 17.974 & & & \\
\hline & ImprvoeEco & 2604.189 & 103 & 25.283 & & & \\
\hline & ImproveGndr & 3050.338 & 103 & 29.615 & & & \\
\hline & ImprovePol & 2936.578 & 103 & 28.510 & & & \\
\hline & ImproveFree & 2996.359 & 103 & 29.091 & & & \\
\hline \multirow[t]{5}{*}{ Total } & ImproveCustom & 2264.750 & 106 & & & & \\
\hline & ImprvoeEco & 4273.000 & 106 & & & & \\
\hline & ImproveGndr & 5017.500 & 106 & & & & \\
\hline & ImprovePol & 4740.750 & 106 & & & & \\
\hline & ImproveFree & 3755.250 & 106 & & & & \\
\hline \multirow{5}{*}{$\begin{array}{l}\text { Corrected } \\
\text { Total }\end{array}$} & ImproveCustom & 2200.540 & 105 & & & & \\
\hline & ImprvoeEco & 2702.585 & 105 & & & & \\
\hline & ImproveGndr & 3064.434 & 105 & & & & \\
\hline & ImprovePol & 4703.899 & 105 & & & & \\
\hline & ImproveFree & 3727.229 & 105 & & & & \\
\hline
\end{tabular}


The ANOVA on the improvement scores in economics status and gender were not significant. Improvement scores in economic status had the following data: $F(2$, $103)=1.946, p>.05$, partial $\eta^{2}=.036$; improvement scores in gender had the following data: $F(2,103)=.238, p>.05$, partial $\eta^{2}=.005$. Table 16 shows a summary of results of a one-way analysis of variance for general improvement scores in five cultural components of CulTests. Details regarding the mean, standard deviation as well as the $95 \%$ confidence intervals for the mean on improvement scores among the three groups can be found in Table 38 (See Appendix P).

Table 17

The Results of Post Hoc Comparisons in Mean Changes for Improvement Scores in Three Cultural Components of CulTests

\begin{tabular}{|c|c|c|c|c|c|c|}
\hline \multirow[t]{2}{*}{ Dependent Variable } & \multirow[t]{2}{*}{$\begin{array}{l}\text { Group (I) - } \\
\text { Group }(\mathrm{J})\end{array}$} & \multirow{2}{*}{$\begin{array}{l}\text { Mean } \\
\text { Difference } \\
(\mathrm{I}-\mathrm{J})\end{array}$} & \multirow{2}{*}{\multicolumn{2}{|c|}{ Std. Error Sig. }} & \multicolumn{2}{|c|}{$\begin{array}{l}95 \% \text { Confidence } \\
\text { Interval }\end{array}$} \\
\hline & & & & & Lower & Upper \\
\hline \multirow[t]{3}{*}{ ImproveCustom } & $(1)-(2)$ & -1.7280 & 1.15465 & .138 & -4.0180 & .5620 \\
\hline & (1)-(3) & $2.5192^{*}$ & 1.01831 & .015 & .4997 & 4.5388 \\
\hline & $(2)-(3)$ & $4.2473^{*}$ & .99377 & .000 & 2.2764 & 6.2182 \\
\hline \multirow[t]{3}{*}{ ImprovePol } & $(1)-(2)$ & -2.5728 & 1.45423 & .080 & -5.4569 & .3113 \\
\hline & $(1)-(3)$ & $6.6250^{*}$ & 1.28251 & .000 & 4.0814 & 9.1686 \\
\hline & (2)-(3) & $9.1978^{*}$ & 1.25160 & .000 & 6.7155 & 11.6801 \\
\hline \multirow[t]{3}{*}{ ImproveFree } & $(1)-(2)$ & 2.2088 & 1.46896 & .136 & -.7045 & 5.1221 \\
\hline & $(1)-(3)$ & $-3.8654^{*}$ & 1.29550 & .004 & -6.4347 & -1.2961 \\
\hline & (2)-(3) & $-6.0742^{*}$ & 1.26428 & .000 & -8.5816 & -3.5668 \\
\hline
\end{tabular}

In order to further determine which teaching methods helped students' make more progress on the aspects of customs and belief, politics and free will, post hoc analysis 
were conducted to evaluate pairwise differences on the improvement scores between CulTestA and CulTestB. Each pairwise comparison was tests at the $.167(.05$ divided by 3 number of comparisons). Table 17 shows the results of post hoc comparisons in mean differences for improvement scores in three cultural components of CulTests. Table 17, illustrates several findings as presented below: improvement scores in custom and belief indicates there was a significant difference between group 1 and group 3, group 2 and group 3 . To be specific, both group 1 and group 2 made significantly more improvements in comparison with group 3. There was no significant difference between group 1 and group 2. In other words, the improvements of group 1 and group 2 were similar; Improvement scores in politics indicate there was a significant difference between group 1 and group 3, group 2 and group 3. To be specific, both group 1 and group 2 made significantly more improvement in comparison with group 3 . There was no significant difference between group 1 and group 2; improvement scores in free will indicate there was a significant difference between group 1 and group 3, group 2 and group 3 . To be specific, both group 1 and group 2 made significant improvements in comparison with group 3. There was no significant difference between group 1 and group 2 .

Summary of findings. The data analytic results above have shown a significant difference among the performance of the three groups of students in terms of their improvement scores on the cultural analysis test in the corresponding three aspects between the pre-test and post-test. To be specific, they included the aspects of customs and belief, politics and free will in the cultural analysis essay. In particular, the two groups instructed by a cultural criticism approach obtained much higher scores in cultural understanding of literary work on the three above mentioned aspects than the group of 
students who only received traditional formalist and biographical teaching. While for the aspects of gender and economic status in the cultural analysis essay, the data analytic results above have not shown a significant difference for the improvement scores among the three groups of students. Therefore, the null hypothesis in Equation (9) is rejected.

\section{Literature Comprehension Tests}

This section compares the experimental impacts of instruction of cultural criticism on students' literature comprehension. Systematic comparisons were performed among the performance of the three groups of students in British literature comprehension tests. This include one repeated-measure mixed-design analysis of covariance (ANCOVA) model and one repeated-measure mixed-design multivariate analysis of covariance (MANCOVA) models. Results of CompTest1, CompTest2, and CompTest3 (defined in the Instruments section of Chapter 3) as well as the three component scores for each CompTest were stored into SPSS program to derive the results in this section.

\section{Repeated-measure Mixed Design ANCOVA for Three CompTests}

The effect of different literature teaching methods on students' literature comprehension was first evaluated by comparing their overall performance on each CompTest over time. The students' performance in the TEM4 may impact differently on the CompTest, and therefore the TEM4 scores were added as covariate in the comparison. The following research questions for the group main effect, the time main effect, and the interaction between group and time were attempted to be answered:

Group main effect: Does each group of students, achieve different average performance scores in three British literature comprehension tests after controlling for the covariate TEM4 scores? 
Null Hypothesis: There is no statistically significant difference for each group of students instructed by different literature teaching methods on average performance scores in British literature comprehension tests, after controlling for the covariate TEM4 scores.

Alternative Hypothesis: There is a statistically significant difference for each group of students instructed by different literature teaching methods on average performance scores in British literature comprehension tests, after controlling for the covariate TEM4 scores.

Statistically, the hypotheses can be represented as

$$
\begin{gathered}
\mathrm{H}_{0}: \mu^{\prime} \text { group } 1=\mu^{\prime} \text { group } 2=\mu^{\prime} \text { group } 3 \\
\mathrm{H}_{\mathrm{a}}: \mu^{\prime} \text { group } i \neq \mu^{\prime} \text { group } k \text { for some } i, k
\end{gathered}
$$

Time main effect: Do students in three groups, on average, achieve different performance scores for British literature comprehension test 1, British literature comprehension test 2, and British literature comprehension test 3, after controlling for the covariate TEM4 scores?

Null Hypothesis: There is no statistically significant difference for all the students on average performance scores for British literature comprehension test 1, British literature comprehension test 2, and British literature comprehension test 3, after controlling for the covariate TEM4 scores.

Alternative Hypothesis: There is a statistically significant difference for all the students on average performance scores for British literature comprehension test 1, British literature comprehension test 2, and British literature comprehension test 3, after controlling for the covariate TEM4 scores. 


$$
\begin{aligned}
& \mathrm{H}_{0}: \mu^{\prime} \text { CompTest } 1=\mu^{\prime} \text { CompTest } 2=\mu^{\prime} \text { CompTest } 3 \\
& \mathrm{H}_{\mathrm{a}}: \mu^{\prime} \text { CompTest } i \neq \mu^{\prime} \text { CompTest } k \text { for some } i, k
\end{aligned}
$$

Interaction between group and time: (a) Do the pairwise differences in the adjusted means of students' performance scores in literature comprehension tests among three groups vary depending on the testing time? (b) Do the pairwise differences in the adjusted means of students' performance scores among three literature comprehension tests vary depending on the groups?

Null Hypothesis: (a) The pairwise differences in the adjusted means of the performance scores for students from the three groups are all the same over different testing time of the literature comprehension tests; (b) The pairwise difference in the adjusted means of the performance scores in the three literature comprehension tests are all the same for students from different groups.

Alternative Hypothesis: (a) The pairwise differences in the adjusted means of the performance scores for students from the three groups are not the same over different testing time of the literature comprehension tests; (b) The pairwise difference in the adjusted means of the performance scores in the three literature comprehension tests are not the same for students from different groups.

The statistical representation is as follows:

$$
\mathrm{H}_{0}:\left\{\begin{array}{l}
\mu_{\text {group 1,Test 1 }}^{\prime}=\mu_{\text {group 1,Test 2 }}^{\prime}=\mu_{\text {group 1,Test 3 }}^{\prime} \\
\mu_{\text {group 2,Test 1 }}^{\prime}=\mu_{\text {group 2,Test 2 }}^{\prime}=\mu_{\text {group 2,Test 3 }}^{\prime} \\
\mu_{\text {group 3,Test 1 }}^{\prime}=\mu_{\text {group 3,Test 2 }}^{\prime}=\mu_{\text {group 3,Test 3 }}^{\prime} \\
\mu_{\text {group 1,Test 1 }}^{\prime}=\mu_{\text {group 2,Test 1 }}^{\prime}=\mu_{\text {group 3,Test 1 }}^{\prime} \\
\mu_{\text {group 1,Test 2 }}^{\prime}=\mu_{\text {group 2,Test 2 }}^{\prime}=\mu_{\text {group 3,Test 2 }}^{\prime} \\
\mu_{\text {group 1,Test 3 }}^{\prime}=\mu_{\text {group 2,Test 3 }}^{\prime}=\mu_{\text {group 3,Test 3 }}^{\prime}
\end{array}\right.
$$




$$
\mathrm{H}_{\mathrm{a}}: \mu^{\prime} \text { group } i, \text { Test } \mathrm{m} \neq \mu^{\prime} \text { group } i, \text { Test } \mathrm{n} \text { OR } \mu^{\prime} \text { group } i, \text { Test } \mathrm{m} \neq \mu^{\prime} \text { group } k \text {, Test } \mathrm{m}
$$

for some group index $i, j$ and some CompTest index $m, n$

A repeated-measure mixed design analysis of covariance (ANCOVA) was conducted to evaluate the effect of different literature teaching methods and length of time for students' performance scores in three British literature comprehension tests. The dependent variables were students' performance scores in each test. The within-subjects factor was time with three levels that have been defined in Table 3, in the Instruments section of Chapter 3. The between-subjects factors were group with three levels that have been defined in Table 1, in the Participants and Settings section of Chapter 3. The covariate was the TEM4 scores. The alpha was set at .05 significance level.

The sphericity was first assumed based on the non-significant result in the Mauchly's Test of Sphericity, $p>.05$. In the Tests of Within-Subjects Effects, the Time main effect was non-significant, $F(2,204)=.319, p>.05$, so the null hypothesis in Equation (13) should not be rejected; The Group $\times$ Time interaction was significant, $F(4,204)=3.576, p=.008<.05$, thus, the null hypothesis in Equation (15) should be rejected; partial $\eta^{2}=.066$, indicated that the interaction of Group and Time effect accounted for $6.6 \%$ of the variance of the CompTest scores. In the Tests of BetweenSubjects Effects, the Group main effect was significant, $F(2,102)=14.787, p<.05$, therefore the null hypothesis in Equation (11) should be rejected; partial $\eta^{2}=.225$, indicated that Group effect accounted for $22.5 \%$ of the variance of the CompTest scores. The relationship between the covariate TEM4 and the dependent variable quiz scores within groups was significant, $F(1,102)=32.339, p<.05$, with the covariate TEM4 accounting for about $24.1 \%$ (i.e., the partial eta is .241) of variance of the CompTest 
scores, while controlling for treatment factor. Table 18 reports a summary of results of repeated measures analysis of covariance mixed design for CompTests.

Table 18

Summary of Results of Repeated Measures Analysis of Covariance Mixed Design for CompTests

\begin{tabular}{lllllll}
\hline Source & $\begin{array}{l}\text { Type III } \\
\text { Sum of } \\
\text { Squares }\end{array}$ & df & $\begin{array}{l}\text { Mean } \\
\text { Square }\end{array}$ & F & Sig. & $\begin{array}{l}\text { Partial Eta } \\
\text { Squared }\end{array}$ \\
\hline Time & 34.459 & 2 & 17.229 & .319 & .727 & .003 \\
Time * Group & 772.105 & 4 & 193.026 & 3.576 & .008 & .066 \\
TEM4 & 5124.941 & 1 & 5124.941 & 32.339 & $<.001$ & .241 \\
Group & 4686.620 & 2 & 2343.310 & 14.787 & $<.001$ & .225 \\
\hline
\end{tabular}

For the adjusted means of average students' performance scores in three CompTests for each group after controlling the influence of TEM 4, group 2 had the largest adjusted mean $(M=70.12)$, group 1 had a smaller adjusted mean $(M=70.12)$, group 3 had the smallest adjusted mean $(M=61.82)$. More details regarding the mean, standard deviation, and the estimated $95 \%$ confidence intervals of mean for group in three CompTests can be found in Table 39 (See Appendix P).

Based on the significant results for the Group effect, follow-up test was conducted to evaluate pairwise differences among the adjusted means of average students' performance scores in three CompTests for each group. There was a significant difference in the adjusted means between the group 1 and group 3, there was also a significant difference in the adjusted means between group 2 and the group 3 ; however, there was no significant difference between group 1 and group 2. The group 1 and group 
2 instructed by a cultural criticism method achieved average higher performance scores in three CompTests than in group 3. The 95\% confidence intervals for the pairwise differences, as well as the adjusted mean difference and standard deviations of mean difference for the three groups, are reported in Table 19.

Table 19

The Results of the Group Main Effect Pairwise Comparison for CompTests

\begin{tabular}{|c|c|c|c|c|c|}
\hline \multirow{2}{*}{$\begin{array}{l}\text { Group (I)- } \\
\text { Group (J) }\end{array}$} & \multirow{2}{*}{$\begin{array}{l}\text { Mean } \\
\text { Difference (I- } \\
\text { J) }\end{array}$} & \multirow[t]{2}{*}{ Std. Error } & \multirow[t]{2}{*}{ Sig. $^{b}$} & \multicolumn{2}{|c|}{$\begin{array}{l}\text { 95\% Confidence Interval for } \\
\text { Difference }^{\mathrm{b}}\end{array}$} \\
\hline & & & & Lower Bound & Upper Bound \\
\hline$\overline{(1)-(2)}$ & -1.361 & 1.981 & .494 & -5.289 & 2.568 \\
\hline (1)-(3) & $6.943^{*}$ & 1.754 & .000 & 3.464 & 10.423 \\
\hline$(2)-(3)$ & $8.304^{*}$ & 1.707 & .000 & 4.919 & 11.689 \\
\hline
\end{tabular}

Based on the significant results for the Group $\times$ Time interaction, two follow-up tests were conducted. The first test evaluated pairwise differences among the adjusted means of students' performance scores in different British literature comprehension tests (CompTest) over time for each group. The second test evaluated pairwise differences among the adjusted means of students' performance scores in different groups for each British literature comprehension test at three time points.

For the first follow-up test, the adjusted mean, standard deviation as well as the 95\% confidence intervals of adjusted mean for each group in different CompTest over three testing time, are reported in Table 40 (See Appendix P). 
Table 20 reports the $95 \%$ confidence intervals for the pairwise differences, as well as the adjusted mean difference and standard deviations of adjusted mean differences in different CompTest over three testing time for each group.

Table 20

The Results of Pairwise Comparisons for Group*Time Interaction in CompTests

\begin{tabular}{|c|c|c|c|c|c|c|}
\hline \multirow[t]{2}{*}{$\overline{\text { Group }}$} & \multirow[t]{2}{*}{$\begin{array}{l}\text { Time(I)- } \\
\text { Time(J) }\end{array}$} & \multirow{2}{*}{$\begin{array}{l}\text { Mean } \\
\text { Difference (I- } \\
\text { J) }\end{array}$} & \multirow[t]{2}{*}{ Std. Error } & \multirow[t]{2}{*}{ Sig.b } & \multicolumn{2}{|c|}{$\begin{array}{l}\text { 95\% Confidence Interval for } \\
\text { Difference }^{\mathrm{b}}\end{array}$} \\
\hline & & & & & \multicolumn{2}{|c|}{ Lower Bound Upper Bound } \\
\hline \multirow[t]{3}{*}{$\overline{1}$} & $(1)-(2)$ & $6.676^{*}$ & 2.256 & .004 & 2.201 & 11.151 \\
\hline & (1)-(3) & 2.076 & 1.908 & .279 & -1.708 & 5.861 \\
\hline & $(2)-(3)$ & $-4.599^{*}$ & 1.947 & .020 & -8.461 & -.737 \\
\hline \multirow[t]{3}{*}{2} & (1)-(2) & $5.026^{*}$ & 2.169 & .022 & .724 & 9.328 \\
\hline & (1)-(3) & -.747 & 1.834 & .685 & -4.386 & 2.891 \\
\hline & $(2)-(3)$ & $-5.773^{*}$ & 1.872 & .003 & -9.487 & -2.060 \\
\hline \multirow[t]{3}{*}{3} & (1)-(2) & $4.927^{*}$ & 1.595 & .003 & 1.764 & 8.090 \\
\hline & (1)-(3) & $-6.197^{*}$ & 1.349 & .000 & -8.873 & -3.522 \\
\hline & (2)-(3) & $-11.124^{*}$ & 1.376 & .000 & -13.855 & -8.394 \\
\hline
\end{tabular}

For group 1, there was a significant difference in the adjusted means between Time 1 and Time 2 as well as between Time 2 and Time 3. But there was no significant difference between Time 1 and Time 3. Students in group 1 achieved around 6.68 higher scores in CompTest1 than in CompTest2, they also achieved around 4.60 higher scores in CompTest3 than in CompTest2.

For group 2, there was a significant difference in the adjusted means between Time 1 and Time 2 as well as between Time 2 and Time 3. But there was no significant 
difference between Time 1 and Time 3. Students in group 2 achieved around 5.03 higher scores in CompTest1 than in CompTest2, they also achieved around 5.77 higher scores in CompTest3 than in CompTest2.

For group 3, there was a significant difference in the adjusted means between Time 1 and Time 2, between Time 2 and Time 3, between Time 1 and Time 3. Students in group 3 achieved around 4.93 higher scores in CompTest1 than in CompTest2, they also achieved around 11.12 higher scores in CompTest 3 than in CompTest2, around 6.20 higher scores in CompTest3 than in CompTest1.

For the second follow-up test, the adjusted mean, standard deviation as well as the 95\% confidence intervals of adjusted mean for students' performance scores in different groups for each CompTest are reported in Table 41 (See Appendix P).

Table 21

The Results of Pairwise Comparisons for Time*Group Interaction in CompTests

\begin{tabular}{|c|c|c|c|c|c|c|}
\hline \multirow[t]{2}{*}{ Time } & \multirow[t]{2}{*}{$\begin{array}{l}\text { Group (I)- } \\
\text { Group (J) }\end{array}$} & \multirow{2}{*}{$\begin{array}{l}\text { Mean } \\
\text { Difference (I- } \\
\text { J) }\end{array}$} & \multirow[t]{2}{*}{ Std. Error } & \multirow[t]{2}{*}{ Sig. ${ }^{b}$} & \multicolumn{2}{|c|}{$\begin{array}{l}\text { 95\% Confidence Interval for } \\
\text { Difference }^{\mathrm{b}}\end{array}$} \\
\hline & & & & & \multicolumn{2}{|c|}{ Lower Bound Upper Bound } \\
\hline \multirow[t]{3}{*}{1} & $(1)-(2)$ & .131 & 2.650 & .961 & -5.126 & 5.387 \\
\hline & $(1)-(3)$ & $10.284^{*}$ & 2.347 & .000 & 5.629 & 14.939 \\
\hline & $(2)-(3)$ & $10.154^{*}$ & 2.283 & .000 & 5.624 & 14.683 \\
\hline \multirow[t]{3}{*}{2} & $(1)-(2)$ & -1.519 & 2.810 & .590 & -7.092 & 4.054 \\
\hline & $(1)-(3)$ & $8.536^{*}$ & 2.488 & .001 & 3.601 & 13.471 \\
\hline & $(2)-(3)$ & $10.055^{*}$ & 2.421 & .000 & 5.253 & 14.856 \\
\hline \multirow[t]{3}{*}{3} & $(1)-(2)$ & -2.693 & 2.207 & .225 & -7.071 & 1.685 \\
\hline & (1)-(3) & 2.011 & 1.955 & .306 & -1.866 & 5.887 \\
\hline & $(2)-(3)$ & $4.704^{*}$ & 1.902 & .015 & .932 & 8.476 \\
\hline
\end{tabular}


Table 21 shows the $95 \%$ confidence intervals for the pairwise differences, as well as the adjusted mean difference and standard deviations of adjusted mean differences for students' performance scores in different groups for each CompTest.

In Time 1, there was a significant difference in the adjusted means between group 1 and group 3 as well as between group 2 and the group 3; however, there was no significant difference between group 1 and group 2. The group 1 and group 2 achieved higher performance scores than group 3 in CompTest1. To be specific, group 1 achieved around 10.28 higher performance scores than group 3, and group 2 achieved around 10.15 higher performance scores than group 3 in CompTest1.

In Time 2, there was a significant difference in the adjusted means between group 1 and group 3, between group 2 and group 3; however, there was no significant difference between group 1 and group 2. The group 1 and group 2 achieved higher performance scores than group 3 in CompTest2. To be specific, group 1 achieved around 8.54 higher performance scores than group 3, and group 2 achieved around 10.06 higher performance scores than group 3 in CompTest2.

In Time 3, there was a significant difference in the adjusted means between group 2 and group 3; however, there was no significant difference in the adjusted means between group 1 and group 3, there was also no significant difference between group 1 and group 2. To be specific, the group 2 achieved 4.70 higher performance scores than group 3 in CompTest3.

Summary of findings. The within subject test indicated that there was a nonsignificant time effect. In other words, there was no difference for students on 
average in the three groups on general performance scores in each CompTest over three testing time. The null hypothesis in Equation (13) cannot be rejected. The between subject test indicated that there was a significant group effect. In other words, there was a difference for each group of students on average performance scores in three CompTests. In particular, the two groups instructed by a cultural criticism approach obtained higher scores in literature comprehension than the group of students who only received traditional formalist and biographical teaching. The results were obtained with the controlling of the potential impact of students' TEM4 scores. Therefore, the null hypothesis in Equation (11) is rejected. The two types of interaction effects were significant. For one thing, given a particular CompTest, the average overall performance from different groups are significantly different. In particular, the groups that received cultural criticism instruction achieved higher scores than the group received traditional instruction method. For another, given a particular group, the students' average overall performance was significantly different across the three CompTests. More specifically, for the two groups under cultural criticism instruction, CompTest 1 and CompTest 3 were not significantly different while they were both significantly different from CompTest2.

\section{Repeated-measure mixed design MANCOVA for Three CompTests}

The effect of different literature teaching methods on students' literature comprehension was then evaluated by comparing their performance on the corresponding three subcategories of each CompTest over time. The students' performance in the TEM4 may impact differently on the CompTest and therefore the TEM4 scores were added as covariate in the comparison. The following research questions for the group main effect, 
the time main effect, and the interaction between group and time were attempted to be answered.

Group main effect: Do students for each group, achieve different average performance scores on cultural and historical contexts, identification of themes, purpose, and plot developments, as well as literary analysis in three British literature comprehension tests, after adjustment of covariate TEM4 scores?

Null Hypothesis: There is no statistically significant difference for each group of students instructed by different literature teaching methods on average performance scores for the aspects of cultural and historical contexts, identification of themes, purpose, and plot developments, as well as literary analysis in British literature comprehension tests, after controlling of covariate TEM4 scores.

Alternative Hypothesis: There is a statistically significant difference for each group of students instructed by different literature teaching methods on average performance scores for the aspects of cultural and historical contexts, identification of themes, purpose, and plot developments, as well as literary analysis in British literature comprehension tests, after controlling of covariate TEM4 scores.

Using $\mu$ ' Group $i, \mathrm{CC}, \mu$ 'Group $i$, ID, and $\mu$ 'Group i, LA to denote the population means of scores for students in Group $i$ at the three components respectively, the hypotheses can be represented as:

$$
\begin{gathered}
\mathrm{H}_{0}:\left\{\begin{array}{l}
\mu_{\text {Group 1,CC }}^{\prime}=\mu_{\text {Group 2,CC }}^{\prime}=\mu_{\text {Group 3,CC }}^{\prime} \\
\mu_{\text {Group 1,ID }}^{\prime}=\mu_{\text {Group 2,ID }}^{\prime}=\mu_{\text {Group 3,ID }}^{\prime} \\
\mu_{\text {Group 1,LA }}^{\prime}=\mu_{\text {Group 2,LA }}^{\prime}=\mu_{\text {Group 3,LA }}^{\prime}
\end{array}\right. \\
\mathrm{H}_{\mathrm{a}}: \mu_{\text {Group } i \mathrm{c} \neq}^{\prime} \mu^{\prime} \text { Group } k \text { c for some } i, k \text { and some component c }
\end{gathered}
$$


Time main effect: Do students in three groups, on average, achieve different average performance scores on cultural and historical contexts, identification of themes, purpose, and plot developments, as well as literary analysis for CompTest1, CompTest2, and CompTest 3 , after controlling of covariate TEM4 scores?

Null Hypothesis: There is no statistically significant difference for all the students on average performance scores in the aspects of cultural and historical contexts, identification of themes, purpose, and plot developments, as well as literary analysis for CompTest1, CompTest2, and CompTest3, after controlling of covariate TEM4 scores.

Alternative Hypothesis: There is a statistically significant difference for all the students on average performance scores in the aspects of cultural and historical contexts, identification of themes, purpose, and plot developments, as well as literary analysis for CompTest1, CompTest 2 and CompTest3, after controlling of covariate TEM4 scores.

Using $\mu^{\prime}$ 'Test $i$, CC, $\mu^{\prime}$ Test $i$, ID , and $\mu^{\prime}$ 'Test i, LA to denote the adjusted population means of scores of CompTest $i$ at the three components respectively, the hypotheses can be represented as:

$$
\mathrm{H}_{0}:\left\{\begin{array}{c}
\mu_{\text {Test 1,CC }}^{\prime}=\mu_{\text {Test 2,CC }}^{\prime}=\mu_{\text {Test 3,CC }}^{\prime} \\
\mu_{\text {Test 1,ID }}^{\prime}=\mu_{\text {Test 2,ID }}^{\prime}=\mu_{\text {Test 3,ID }}^{\prime} \\
\mu_{\text {Test 1,LA }}^{\prime}=\mu_{\text {Test 2,LA }}^{\prime}=\mu_{\text {Test 3,LA }}^{\prime}
\end{array}\right.
$$

$\mathrm{H}_{\mathrm{a}}: \mu^{\prime}$ 'Test $i c \neq \mu^{\prime}$ 'Test $k \mathrm{c}$ for some $i, k$ and some component $\mathrm{c}$

Interaction between group and time: (a) Do the pairwise differences in the adjusted means of students' performance scores in three subcategories of literature comprehension tests among three groups vary depending on the testing time? (b) Do the pairwise differences in the adjusted means of students' performance scores in three subcategories of three literature comprehension tests vary depending on the groups? 
Null Hypothesis: (a) The pairwise differences in the adjusted means of the performance scores in three subcategories of literature comprehension tests for students from the three groups are all the same over different testing time; (b) The pairwise difference in the adjusted means of the performance scores in the three subcategories of three literature comprehension tests are all the same for students from different groups.

Alternative Hypothesis: (a) The pairwise differences in the adjusted means of the performance scores in three subcategories of literature comprehension tests for students from the three groups are not the same over different testing time; (b) The pairwise difference in the adjusted means of the performance scores in the three subcategories of three literature comprehension tests are not the same for students from different groups.

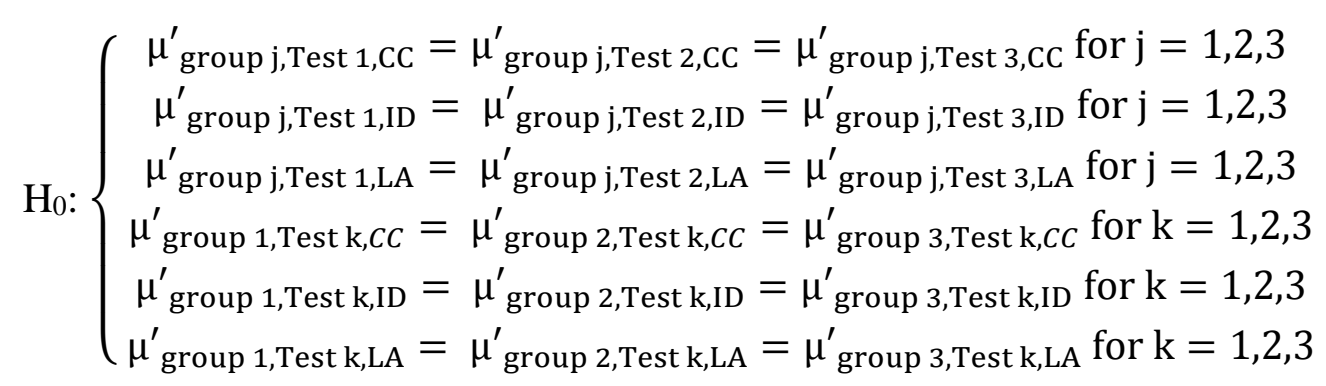

$$
\mathrm{H}_{\mathrm{a}} \text { : at least one of above does not hold }
$$

A repeated-measure mixed design Multivariate Analysis of Covariance (MANCOVA) was conducted to evaluate the effect of different literature teaching methods and students' performance scores in three subcategories of British literature comprehension tests over three testing time. The dependent variables were students' normalized performance scores for three subcategories of each CompTest, that have been defined in Table 3, in the Instruments section of Chapter 3. The within-subjects factor was time with three levels that have been defined in Table 3, in the Instruments section of 
Chapter 3. The between-subjects factors were group with three levels, that have been defined in Table 1, in the Participants and Settings section of Chapter 3. The alpha was set at .05 significance level.

Details regarding the mean and standard deviations for each group in three subcategories of CompTests can be found in Table 42 (See Appendix P). As the result in the Box's test was significant, $F(90,17130.97)=1.479, p=.002<.05$, the Time main effect, Group effect, Group $\times$ Time interaction effect as well as covariate effect were tested using the multivariate criterion of Pillai's Trace $(\vee)$.

Table 22

Summary of Results of Repeated Measures Multivariate Analysis Mixed Design of Covariance for CompTests

\begin{tabular}{llllllll}
\hline Effect & & Value & F & Hypothesis df & Error df & Sig. & $\begin{array}{l}\text { Partial Eta } \\
\text { Squared }\end{array}$ \\
\hline Between & TEM4 & .229 & $9.929^{\mathrm{b}}$ & 3.000 & 100.000 & $<.001$ & .229 \\
Subjects & group & .401 & 8.431 & 6.000 & 202.000 & $<.001$ & .200 \\
& Time & .089 & $1.583^{\mathrm{b}}$ & 6.000 & 97.000 & .160 & .089 \\
Within & Subjects & Time * group & .363 & 3.616 & 12.000 & 196.000 & $<.001$ \\
\hline
\end{tabular}

The Time main effect was nonsignificant, $F(6,97)=1.583, p=.160>.05$, partial $\eta^{2}=.089$, so the null hypothesis in Equation (19) should not be rejected. The Group main effect was significant, $F(6,202)=8.431, p<.05$, partial $\eta^{2}=.200$, so the null hypothesis in Equation (17) should be rejected. The Group $\times$ Time interaction was also significant, $F(12,196)=3.616, p<.05$, partial $\eta^{2}=.181$, so the null hypothesis in Equation (21) should be rejected. The relationship between the covariate TEM4 and the dependent variables within groups was significant, $F(3,100)=9.929, p=.000<.05$, with the covariate TEM4 
accounting for about $22.9 \%$ (i.e., the partial eta is .229) of variance of the students' performance scores in three subcategories of CompTests, while controlling for the treatment factor. A summary of results of repeated measures mixed-design multivariate analysis of covariance for CompTests is reported in Table 22.

Based on the significant results for the Group effect, a follow-up test was conducted to evaluate pairwise differences among the adjusted average means of students' performance scores in three subcategories of all CompTests for each group. More details regarding the adjusted average mean, standard deviation as well as the $95 \%$ confidence intervals of adjusted average mean for each group in three subcategories of all CompTests over three testing time for each group can be found in Table 43 (See Appendix P).

Table 23 shows the results of the group main effect pairwise comparison for three subcategories in three CompTests.

For the section of Cultural and Historical Contexts (CC), there was a significant difference in the adjusted means between group 2 and group 3, there was no significant difference in the adjusted means between group 1 and the group 3, between group 1 and group 2. The group 2 achieved 6.11 higher performance scores in $\mathrm{CC}$ for three CompTests than group 3.

For the section of Identification of Purpose, Themes and Plot Developments (ID), there was no significant difference in the adjusted means among group 1, group 2 and group 3.

For the section of Literary Analysis (LA), there was a significant difference in the adjusted means between group 2 and group 3, between group 1 and group 3. However, 
there was no significant difference between group 1 and group 2. The group 1 achieved around 13.50 and 12.34 higher performance scores in LA for three CompTests than in group 1 and group 3 respectively.

Table 23

The Results of the Group Main Effect Pairwise Comparison for Three Subcategories in CompTests

\begin{tabular}{lllllll}
\hline Measure & $\begin{array}{l}\text { Group (I)- Mean } \\
\text { Group (J) }\end{array}$ & $\begin{array}{l}\text { Difference (I- } \\
\text { J) }\end{array}$ & & & & \multicolumn{2}{l}{$\begin{array}{l}\text { Std. Error } \\
\text { for Difference }\end{array}$} & Sig. $^{\mathrm{b}}$ \\
\hline CC & $(1)-(2)$ & -4.581 & 2.375 & .057 & -9.292 & .130 \\
& $(1)-(3)$ & 1.531 & 2.103 & .468 & -2.641 & 5.703 \\
& $(2)-(3)$ & $6.112^{*}$ & 2.046 & .004 & 2.053 & 10.171 \\
ID & $(1)-(2)$ & -4.773 & 2.660 & .076 & -10.049 & .504 \\
& $(1)-(3)$ & -.558 & 2.356 & .813 & -5.231 & 4.115 \\
& $(2)-(3)$ & 4.215 & 2.292 & .069 & -.332 & 8.761 \\
LA & $(1)-(2)$ & 1.156 & 2.652 & .664 & -4.104 & 6.417 \\
& $(1)-(3)$ & $13.497^{*}$ & 2.349 & .000 & 8.838 & 18.155 \\
& $(2)-(3)$ & $12.340^{*}$ & 2.285 & .000 & 7.808 & 16.873 \\
\hline
\end{tabular}

Based on the significant results for the Group $\times$ Time interaction, two follow-up tests were conducted. The first test evaluated pairwise differences among the adjusted means of students' performance scores in three subcategories of different British literature comprehension tests over time for each group. The second test evaluated pairwise differences among the adjusted means of students' performance scores in three subcategories of each British literature comprehension test in different groups. 
Table 24

The Results of Pairwise Comparisons for Group *Time Interaction in Three Subcategories of CompTests

\begin{tabular}{|c|c|c|c|c|c|c|c|}
\hline \multirow[t]{2}{*}{ Measure } & \multirow[t]{2}{*}{ Group } & \multirow[t]{2}{*}{$\begin{array}{l}\text { Time(I)- } \\
\text { Time(J) }\end{array}$} & \multirow{2}{*}{$\begin{array}{l}\text { Mean } \\
\text { Difference (I- } \\
\text { J) }\end{array}$} & \multirow{2}{*}{\multicolumn{2}{|c|}{ Std. Error Sig. ${ }^{\mathrm{b}}$}} & \multicolumn{2}{|c|}{$\begin{array}{l}\text { 95\% Confidence Interval for } \\
\text { Difference }^{\mathrm{b}}\end{array}$} \\
\hline & & & & & & Lower Bound & Upper Bound \\
\hline \multirow[t]{9}{*}{$\overline{\mathrm{CC}}$} & \multirow[t]{3}{*}{1} & $(1)-(2)$ & $16.848^{*}$ & 3.725 & $<.001$ & 9.459 & 24.237 \\
\hline & & $(1)-(3)$ & 4.976 & 3.251 & .129 & -1.472 & 11.424 \\
\hline & & $(2)-(3)$ & $-11.872^{*}$ & 3.522 & .001 & -18.857 & -4.886 \\
\hline & \multirow[t]{3}{*}{2} & $(1)-(2)$ & 2.955 & 3.582 & .411 & -4.149 & 10.059 \\
\hline & & $(1)-(3)$ & -.957 & 3.126 & .760 & -7.157 & 5.242 \\
\hline & & $(2)-(3)$ & -3.912 & 3.386 & .251 & -10.628 & 2.804 \\
\hline & \multirow[t]{3}{*}{3} & $(1)-(2)$ & $11.523^{*}$ & 2.634 & $<.001$ & 6.300 & 16.747 \\
\hline & & $(1)-(3)$ & 3.604 & 2.298 & .120 & -.954 & 8.163 \\
\hline & & $(2)-(3)$ & $-7.919^{*}$ & 2.490 & .002 & -12.857 & -2.981 \\
\hline \multirow[t]{9}{*}{ ID } & \multirow[t]{3}{*}{1} & $(1)-(2)$ & $-16.476^{*}$ & 3.424 & $<.001$ & -23.268 & -9.685 \\
\hline & & $(1)-(3)$ & 3.116 & 3.606 & .389 & -4.036 & 10.268 \\
\hline & & $(2)-(3)$ & $19.593^{*}$ & 3.331 & $<.001$ & 12.987 & 26.199 \\
\hline & \multirow[t]{3}{*}{2} & $(1)-(2)$ & $-14.031^{*}$ & 3.292 & $<.001$ & -20.560 & -7.501 \\
\hline & & $(1)-(3)$ & 5.962 & 3.467 & .088 & -.914 & 12.838 \\
\hline & & $(2)-(3)$ & $19.993^{*}$ & 3.202 & $<.001$ & 13.641 & 26.344 \\
\hline & \multirow[t]{3}{*}{3} & $(1)-(2)$ & -3.245 & 2.421 & .183 & -8.046 & 1.556 \\
\hline & & $(1)-(3)$ & 4.270 & 2.549 & .097 & -.786 & 9.326 \\
\hline & & $(2)-(3)$ & $7.515^{*}$ & 2.354 & .002 & 2.845 & 12.185 \\
\hline \multirow[t]{9}{*}{ LA } & \multirow[t]{3}{*}{1} & $(1)-(2)$ & $13.911^{*}$ & 3.926 & .001 & 6.125 & 21.698 \\
\hline & & $(1)-(3)$ & 1.056 & 3.560 & .767 & -6.005 & 8.117 \\
\hline & & $(2)-(3)$ & $-12.855^{*}$ & 3.720 & .001 & -20.233 & -5.478 \\
\hline & \multirow[t]{3}{*}{2} & $(1)-(2)$ & $15.654^{*}$ & 3.774 & $<.001$ & 8.168 & 23.140 \\
\hline & & $(1)-(3)$ & $-7.037^{*}$ & 3.423 & .042 & -13.826 & -.248 \\
\hline & & $(2)-(3)$ & $-22.691^{*}$ & 3.576 & $<.001$ & -29.784 & -15.598 \\
\hline & \multirow[t]{3}{*}{3} & $(1)-(2)$ & $5.615^{*}$ & 2.775 & .046 & .111 & 11.120 \\
\hline & & $(1)-(3)$ & $-16.470^{*}$ & 2.517 & $<.001$ & -21.461 & -11.478 \\
\hline & & $(2)-(3)$ & $-22.085^{*}$ & 2.629 & $<.001$ & -27.301 & -16.869 \\
\hline
\end{tabular}


For the first test of pairwise comparisons, details regarding the adjusted mean, standard deviation as well as the $95 \%$ confidence intervals of adjusted mean for each group in different CompTest over three testing time can be found in Table 44 (See Appendix P).

The $95 \%$ confidence intervals for the pairwise differences, as well as the adjusted mean difference and standard deviations of adjusted mean differences on three subcategories of different CompTest over three testing time for each group are reported in Table 24.

In the section of Cultural and Historical Context (CC), for group 1, there was a significant difference in the adjusted means between Time 1 and Time 2 , between Time 2 and Time 3 . To be specific, for group 1, Time 1 has a significantly higher score than Time 2, Time 3 has a significantly higher score than Time 2. Students in group 1 achieved around 16.85 higher scores for $\mathrm{CC}$ in CompTest1 than in CompTest2, they also achieved around 11.87 higher scores for $\mathrm{CC}$ in CompTest 3 than in CompTest2. But there was no significant difference between Time 1 and Time 3. For group 2, there was not a significant difference in the adjusted means between Time 1 and Time 2, between Time 1 and Time 3, and between Time 2 and Time 3. For group 3, there was a significant difference in the adjusted means between Time 1 and Time 2, between Time 2 and Time 3. To be specific, for group 3, Time 1 has a significantly higher score than Time 2, Time 3 has a significantly higher score than Time 2 . Students in group 3 achieved around 11.52 higher scores for $\mathrm{CC}$ in CompTest1 than in CompTest2, they also achieved around 7.92 higher scores for $\mathrm{CC}$ in CompTest3 than in CompTest2. But there was no significant difference between Time 1 and Time 3. 
In the section of Identification of Purpose, Themes and Plot Developments (ID), for group 1, there was a significant difference in the adjusted means between Time 1 and Time 2, between Time 2 and Time 3. But there was no significant difference between Time 1 and Time 3 . To be specific, for group 1, Time 1 has a significantly higher score than Time 2, Time 2 has a significantly higher score than Time 3 . Students in group 1 achieved around 16.48 higher scores for ID in CompTest 2 than in CompTest 1 , they also achieved around 19.59 higher scores for ID in CompTest2 than in CompTest3. For group 2, there was a significant difference in the adjusted means between Time 1 and Time 2, there was also a significant difference in the adjusted means between Time 2 and Time 3 . But there was no significant difference between Time 1 and Time 3. To be specific, for group 2, Time 2 has a significantly higher score than Time 1 , Time 2 has a significantly higher score than Time 3. Students in Group 2 achieved around 14.03 higher scores for ID in CompTest2 than in CompTest1, they also achieved around 19.99 higher scores for ID in CompTest2 than in CompTest3. For group 3, there was a significant difference in the adjusted means between Time 2 and Time 3 . But there was no significant difference between Time 1 and Time 3. There was also no significant difference between Time 1 and Time 2. To be specific, for group 3, Time 2 has a significantly higher score than Time 3. Students in group 3 achieved around 7.52 higher scores for ID in CompTest2 than in CompTest3.

In the section of Literary Analysis (LA), for group 1, there was a significant difference in the adjusted means between Time 1 and Time 2, between Time 2 and Time 3. But there was no significant difference between Time 1 and Time 3. To be specific, for group 1, Time 1 has a significantly higher score than Time 2, Time 3 has a significantly 
higher score than Time 2. Students in group 1 achieved around 13.91 higher scores for LA in CompTest 1 than in CompTest2, they also achieved around 12.86 higher scores for LA in CompTest3 than in CompTest2. For group 2, there was a significant difference in the adjusted means between Time 1 and Time 2, between Time 2 and Time 3 as well as Time 1 and Time 3.

To be specific, for group 2, Time 1 has a significantly higher score than Time 2 , Time 3 has a significantly higher score than Time 1 and Time 2 . Students in group 2 achieved around 15.65 higher scores for LA in CompTest1 than in CompTest2, they achieved around 7.04 higher scores for LA in CompTest3 than in CompTest1. They also achieved around 22.69 higher scores for LA in CompTest 3 than in CompTest2.

For group 3, there was a significant difference in the adjusted means between Time 1 and Time 2, between Time 2 and Time 3 as well as between Time 1 and Time 3 . To be specific, for group 3, Time 1 has a significantly higher score than Time 2, Time 3 has a significantly higher score than Time 1 and Time 2 . Students in group 3 achieved around 5.62 higher scores for LA in CompTest1 than in CompTest2, they achieved around 16.47 higher scores for LA in CompTest3 than in CompTest1. They also achieved around 22.09 higher scores for LA in CompTest3 than in CompTest2.

For the second test of the pairwise comparison, details regarding the adjusted mean, standard deviation as well as the $95 \%$ confidence intervals of adjusted mean for students' performance scores of three subcategories of each CompTest in different groups can be found in Table 45 (See Appendix P). 
Table 25

The Results of Pairwise Comparisons for Time ${ }^{*}$ Group Interaction in Three Subcategories of CompTests

\begin{tabular}{|c|c|c|c|c|c|c|c|}
\hline Measure & Time & $\begin{array}{l}\text { Group(I)- } \\
\text { Group(J) }\end{array}$ & $\begin{array}{l}\text { Mean } \\
\text { Difference (I- } \\
\text { J) }\end{array}$ & Std. Error & Sig. ${ }^{b}$ & \multicolumn{2}{|c|}{$\begin{array}{l}95 \% \text { Confidence Interval } \\
\text { for Difference } \\
\text { Lower Bound Upper Bound }\end{array}$} \\
\hline \multirow[t]{9}{*}{$\mathrm{CC}$} & \multirow[t]{3}{*}{1} & $(1)-(2)$ & 2.028 & 3.582 & .573 & -5.077 & 9.132 \\
\hline & & $(1)-(3)$ & 3.763 & 3.172 & .238 & -2.528 & 10.055 \\
\hline & & $(2)-(3)$ & 1.735 & 3.086 & .575 & -4.386 & 7.857 \\
\hline & \multirow[t]{3}{*}{2} & $(1)-(2)$ & $-11.865^{*}$ & 4.048 & .004 & -19.894 & -3.836 \\
\hline & & $(1)-(3)$ & -1.561 & 3.585 & .664 & -8.671 & 5.549 \\
\hline & & $(2)-(3)$ & $10.304^{*}$ & 3.488 & .004 & 3.386 & 17.221 \\
\hline & \multirow[t]{3}{*}{3} & $(1)-(2)$ & -3.906 & 3.363 & .248 & -10.575 & 2.764 \\
\hline & & $(1)-(3)$ & 2.391 & 2.978 & .424 & -3.515 & 8.298 \\
\hline & & $(2)-(3)$ & $6.297^{*}$ & 2.897 & .032 & .550 & 12.044 \\
\hline \multirow[t]{9}{*}{ ID } & \multirow[t]{3}{*}{1} & $(1)-(2)$ & -6.537 & 3.644 & .076 & -13.765 & .692 \\
\hline & & $(1)-(3)$ & -5.353 & 3.227 & .100 & -11.754 & 1.048 \\
\hline & & $(2)-(3)$ & 1.184 & 3.140 & .707 & -5.044 & 7.412 \\
\hline & \multirow[t]{3}{*}{2} & $(1)-(2)$ & -4.091 & 3.684 & .269 & -11.398 & 3.217 \\
\hline & & $(1)-(3)$ & $7.878^{*}$ & 3.263 & .018 & 1.407 & 14.350 \\
\hline & & $(2)-(3)$ & $11.969^{*}$ & 3.174 & $<.001$ & 5.673 & 18.266 \\
\hline & \multirow[t]{3}{*}{3} & $(1)-(2)$ & -3.691 & 4.161 & .377 & -11.944 & 4.563 \\
\hline & & $(1)-(3)$ & -4.199 & 3.685 & .257 & -11.508 & 3.110 \\
\hline & & $(2)-(3)$ & -.508 & 3.585 & .888 & -7.620 & 6.603 \\
\hline \multirow[t]{9}{*}{ LA } & \multirow[t]{3}{*}{1} & $(1)-(2)$ & 3.273 & 4.283 & .447 & -5.222 & 11.769 \\
\hline & & $(1)-(3)$ & $22.104^{*}$ & 3.793 & $<.001$ & 14.580 & 29.627 \\
\hline & & $(2)-(3)$ & $18.831^{*}$ & 3.691 & $<.001$ & 11.510 & 26.151 \\
\hline & \multirow[t]{3}{*}{2} & $(1)-(2)$ & 5.016 & 4.215 & .237 & -3.345 & 13.376 \\
\hline & & $(1)-(3)$ & $13.808^{*}$ & 3.733 & $<.001$ & 6.404 & 21.211 \\
\hline & & $(2)-(3)$ & $8.792^{*}$ & 3.632 & .017 & 1.589 & 15.996 \\
\hline & \multirow[t]{3}{*}{3} & $(1)-(2)$ & -4.820 & 3.440 & .164 & -11.643 & 2.003 \\
\hline & & $(1)-(3)$ & 4.578 & 3.046 & .136 & -1.464 & 10.620 \\
\hline & & $(2)-(3)$ & $9.398^{*}$ & 2.964 & .002 & 3.519 & 15.277 \\
\hline
\end{tabular}


Table 25 shows the $95 \%$ confidence intervals for the pairwise differences, as well as the adjusted mean difference and standard deviations of adjusted mean differences for students' performance scores in different groups for three subcategories of each CompTest.

For the Section of Cultural and Historical Contexts, in Time 1 when CompTest1 was administrated, there were no significant difference in the adjusted means between group 1 and group 2, group 2 and group 3 as well as group 1 and group 3 respectively.

In Time 2 when CompTest 2 was administrated, there was a significant difference in the adjusted means between group 1 and group 2, as well as between group 2 and group 3; however, there was no significant difference between group 1 and group 3 . The group 2 instructed by a cultural criticism method for the second time achieved around 11.87 and 10.30 higher performance scores than the group 1 instructed by a cultural criticism method for the first time and the group 3 instructed by traditional formalist and biographical method in CompTest2.

In Time 3 when CompTest 3 was administrated, there was a significant difference in the adjusted means between group 2 and group 3; however, there was no significant difference in the adjusted means between the group 1 and group 2 as well as between group 1 and group 3 . The group 2 taught by a cultural criticism approach second around 6.30 higher performance scores than group 3 instructed by traditional formalist and biographical method in CompTest3.

For the Section of Identification of Purpose, Themes and Plot Developments in Time 1 when CompTest1 was administrated, there were no significant difference in the 
adjusted means between group 1 and group 2, group 2 and group 3 as well as group 1 and group 3 respectively.

In Time 2 when CompTest 2 was administrated, there was a significant difference in the adjusted means between group 1 and group 3 , there was also a significant difference in the adjusted means between group 2 and group 3; however, there was no significant difference between group 1 and group 2. The groups (include group 1 and group 2) instructed by cultural criticism method achieved higher performance scores than the group 3 instructed by traditional formalist and biographical method in CompTest2. To be specific, group 1 achieved around 7.88 higher performance scores than the group 3; group 2 achieved around 11.97 higher performance scores than group 3.

In Time 3 when CompTest 3 was administrated, there were no significant difference in the adjusted means between group 1 and group 2, group 2 and group 3 as well as group 1 and group 3 respectively.

For the Section of Literary Analysis (LA), in Time 1 when CompTest1 was administrated, there was a significant difference in the adjusted means between group 1 and group 3, there was also a significant difference in the adjusted means between group 2 and group 3; however, there was no significant difference between group 1 and group 2. The groups (include group 1 and group 2 ) instructed by a cultural criticism method achieved average higher performance scores than the group 3 instructed by traditional formalist and biographical method in CompTest1. To be specific, group 1 achieved around 22.10 higher performance scores than the group 3; group 2 achieved around 18.83 higher performance scores than group 3. 
In Time 2 when CompTest 2 was administrated, there was a significant difference in the adjusted means between group 1 and group 3, there was also a significant difference in the adjusted means between group 2 and group 3; however, there was no significant difference between group 1 and group 2. The groups (include group 1 and group 2) instructed by a cultural criticism method achieved average higher performance scores than the group 3 instructed by traditional formalist and biographical method in CompTest2. To be specific, group 1 achieved around 13.81 higher performance scores than the group 3; group 2 achieved around 8.79 higher performance scores than group 3 .

In Time 3 when CompTest 3 was administrated, there was a significant difference in the adjusted means between group 2 and group 3; however, there was no significant difference in the adjusted means between the group 1 and group 2; there was also no significant difference between group 1 and group 3 . The group 2 instructed by a cultural criticism method for the second trial achieved around 9.40 higher performance scores than the group 3 instructed by traditional formalist and biographical method in CompTest3.

Summary of findings. The within subject test indicated that there was a nonsignificant time effect. In other words, there was no significant difference for students on average in the three groups on performance scores for three subcategories in each CompTest over three testing times. One cannot reject the null hypothesis in Equation (19). The between subject test indicated that there was a significant group effect. In other words, there was a difference for each group of students on average performance scores for three subcategories in three CompTests. In particular, the group 2 instructed by a cultural criticism method obtained much better overall literature comprehension than the 
group 3 that received traditional formalist and biographical teaching on the aspect of cultural and historical contexts; the group 1 and group 2 both instructed by a cultural criticism method obtained much better overall literature comprehension than the group 3 that received traditional formalist /biographical instruction on the aspect of literary analysis. The results were obtained after controlling of the potential impact of students' TEM4 scores. Therefore, the null hypothesis in Equation (17) is rejected. The two types of interaction effects were significant. This rejects the overall hypothesis in Equation (21). A first remarkable observation was that students from the same group didn't demonstrate stable performance across the three tests. This statement held for all the three components in the CompTest. A second observation was that students who received cultural criticism instruction have never performed worse than students who received traditional formalist and biographical instruction in any of the components in CompTests. Cultural criticism in those sets of experiments either performed significantly better scores or about the same. Regarding the subcategory of cultural and historical contexts, the difference between the two methods become significant at the $2^{\text {nd }}$ and $3^{\text {rd }}$ CompTest where students that received cultural criticism instruction performed significantly better than the group under traditional instruction method. Regarding the subcategory of identification of themes, purpose, and plot developments, only in CompTest 2 have the results shown significant difference in the performance between students instructed by a cultural criticism method and students instructed by a traditional method. The former had an average better performance than the latter. Regarding the subcategory of literary analysis, students received cultural criticism instruction performed significantly better 
than the group received traditional formalist and biographical instruction in all the three CompTests. 


\section{CHAPTER V}

\section{DISCUSSION AND RECOMMENDATION}

This chapter concludes the study from three perspectives. First, it summarizes the findings and provides a conclusive answer to the research questions that were presented in the Research Questions Section of Chapter 3. Second, it summarizes the practical and theoretical limitations of this study. Finally, a list of future directions, the possible follow-up studies as well as practical implications are presented.

\section{Discussion of Findings}

Cultural criticism is a critical approach that considers influences that readers bring to their engagement with a given literary text (Gunn, 1987). The fundamental focus of this study was investigating the relative effectiveness of utilizing the cultural criticism approach for cross-cultural literature learners in the comparison of traditional formalist and biographical method. In particular, the target population of interest for this study were students with sufficient English language proficiencies who are reading English literary texts from cultures other than their own. In Chapter 1, two research questions were introduced that concentrated on comparing the cultural criticism approach with the traditional formalist and biographical method from the aspect of students' cultural understanding of literary texts as well as their literature comprehension. In the section of Research Design in Chapter 3, a quasi-experimental design was used to formally study the effect from a cultural understanding perspective. In the section of Research Design in Chapter 3, a repeated measure mixed design has also been applied to study the effect from literature comprehension perspective. Those experimental designs investigated the following aspects: (a) whether the cultural criticism teaching method helped students 
achieve better general performance scores and component scores in tests; (b) whether the cultural criticism teaching method helped students make greater improvements on general performance scores and component scores in tests. While experimental results have been extensively discussed in Chapter 4, here the research findings are summarized.

\section{Relative Effectiveness of Cultural Criticism on students' Cultural Understanding}

As seen in the Cultural Analysis Tests section of Chapter 4, the students instructed through the lens of cultural criticism demonstrated significantly higher performance scores in the post-test of cultural understanding of literary texts than students who received the instruction through traditional formalist and biographical criticism. The advantage is reflected in all the five identified components of cultural analysis: (a) customs and beliefs towards marriage (b) gender roles concerning marriage; (c) economic status concerning marriage; (d) politics concerning marriage; and (e) free will concerning marriage. The quantitative results are statistically significant either with (Chapter 4, section of Simple Main Group Effect Test) or without (Chapter 4, section of One-way ANOVA for Post-test CulTestB) the consideration of students' prior skills literature understanding. Another observation showed the fact that in the two experimental groups, students taught by a cultural criticism approach second performed higher in the post-test of cultural understandings of literary works than students taught by a cultural criticism approach first.

Regarding students' improvement in cultural understanding, students in the class who were taught through a cultural criticism approach demonstrated significantly more improvements in cultural understanding of literary work when they were compared with students who were taught through traditional formalist and biographical teaching. More 
specifically, among the five identified components in cultural analysis, students who engaged cultural criticism have shown significant improvement in understanding the components of customs and belief, politics and free will towards marriage.

\section{Relative Effectiveness of Cultural Criticism on students' Literature Comprehension}

As seen in the Literature Comprehension Tests section of Chapter 4, after controlling the covariate scores of TEM-4 (the nationwide standard evaluation of English major students' knowledge and skills in English language and literature), students who were instructed through cultural criticism method had higher performance scores in comprehending British literature than students who received the instruction through traditional formalist and biographical criticism. Furthermore, the advantage was reflected in two of the total three components, that is, cultural and historical contexts as well as literary analysis. The cultural criticism instruction has not shown significant advantage in the component of the identification of themes, purposes, and plot development, to be specific, students who were instructed through cultural criticism method only had significantly higher performance scores in the above component in one of the three comprehension tests than students who received the traditional instruction. On instructor's different levels of proficiency in using a cultural criticism approach, there was no significant difference in performance scores between students taught by a cultural criticism approach first and students taught by a cultural criticism second. The students taught by a cultural criticism approach second only had significantly higher performance scores on cultural and historical context than students taught by a cultural criticism approach first in British Literature Comprehension Test 2. 
In addition, although students' performance varied across the three comprehension tests, it is not evident that students using cultural criticism approach made continuous progress over time. It might be due to the different levels of coefficient of difficulty in three designed literature comprehension tests.

\section{Discussions}

Results of this study have shown that students who received instruction through a cultural criticism lens had significantly higher scores in the related tests of cultural understandings of literary works and in the literature comprehension tests than students who received the instruction through traditional formalist and biographical criticism. To some extent, the research provided empirical evidence in supporting the effect of employing a cultural criticism approach in the classroom settings engaged in crosscultural literature teaching and learning. The traditional formalist and biographical criticism contended an objective and pre-determined nature of the meaning of literary texts (Karolides, 2000), these approaches focused on details about the author, historical context and literary mechanics to analyze literature (Li, 1998. Hence using these approaches in the teaching and learning cross-cultural literature in China might alienate the students and the contexts of the text, and it further prevent students from cultural understanding of literature works and being engaged in literature comprehension.

Cultural criticism, as a critical approach, considers influences that readers bring to their engagement with a given literary text (Gunn,1987). The findings of the research supported that the effectiveness of a developed pedagogy of cultural criticism based on the theoretical framework that are comprised of four theories (Reader-response theory, cultural schemata theory, theory of interpretative communities, and theory of cultural 
criticism) for Chinese undergraduate English majors, more details on the four theories could be found in Theoretical Framework section of Chapter 2. Overall, the four theories influenced the approaches that the researcher implemented the cultural criticism instruction into the teaching practice. Influenced by the lens of cultural schemata theory, students need to understand their own cultures first by reviewing their own frames of reference prior to understanding the foreign culture within the texts; influenced by readerresponse theory (Rosenblatt, 1968) and cultural criticism theory (Gunn, 1987), students were required to compare or contrast the unfamiliar culture with their own cultural context, and thus students experienced multiple contexts as a means of reflecting on the complexity of the world, finally they readjusted their worldviews to enhance cultural understandings of literary work (Kentner, 2005); influenced by interpretative communities theory (Fish, 1982), students were engaged in cultural criticism through the act of discussions in their learning communities. In the classroom setting that students who received instruction through a cultural criticism lens in this study, the use of a cultural criticism approach enabled students there to comprehend the culture depicted in the text, actively construct social and cultural meaning behind the text, identify the gaps between the cultural knowledge they have already known and the cultural referents within the original texts, and reshape the worldviews towards the British culture in the literature classes. Moreover, students shared their diverse perspectives within a particular learning community, and reasoned the difference in the values, beliefs, and attitudes among different cultures within the cultural context of a particular text through intensive discussions and inquiry of personal, social, cultural and textual matters in class (Moran, 2001). In this way, their abilities in comprehension of literary works were developed. 


\section{Limitations of Study}

This study has several limitations in terms of the instruments, the testing time and the sample.

The instruments in this research were two cultural analysis essay tests and three British literature comprehension tests. The instruments used in this study assisted the researcher in investigating two main research questions that focus on students' cultural understanding of literary works and their literature comprehension. The validity of the instruments was obtained through collaboration with experts in the related field and peer review for assessing the content appropriateness. For the evaluation on students' cultural understanding of a literary work, the researcher designed two essay tests adapted from an online published assignment for a cultural analysis essay, and co-developed the scoring rubric with FIU professors in English Education as well as in English Language and Literature. The panel reviewed the content appropriateness of the designed essays for assessing cultural understanding of literary works. The professors also participated in developing the rubric; and their expertise ensured that their scoring of the rubric could accurately evaluate the quality of students' cultural understanding of literary works. In this way, the internal validity of the instrument for assessing students' cultural understanding could be guaranteed. However, there were no published validity estimates for the instruments that were used to collect data. This was one limitation of the study. If the researcher was able to relate to published validity estimates, the instruments would be more authoritative measures with which to assess students' cultural understanding. For the assessment on students' literature comprehension, all of the three British literature comprehension tests were developed and self-organized by the researcher from a variety 
of standardized English test bank. The internal validity of the instruments was assessed through peer review. The questions were selected from multiple sources and the selection criterion of these questions was primarily based on the appropriateness of the content for each test. This has resulted in some variation in the level of difficulty among the three tests. To be specific, students' performance scores were distributed unevenly among three tests. In particular, Test 2 turned out to be the most difficult test and students all had poor performance in that test regardless of which teaching method has been applied. This reflected the lack of the reliability for assessing students' literature comprehension. If the researcher included published reliability estimates for the instruments, the instrument design could be more consistent and more sound.

Another limitation was the time when CompTest1 was administrated. The general linear model of repeated measures mixed design evaluates the behavior of the dependent variable of the same testing object over time or at different testing conditions. Theoretically, it is preferred that the treatment is not assigned to the testing objects at the first test condition or for the first testing time. The respective measurement can be used as a baseline and be further compared with measurement in other testing conditions or times. In this research, on one hand, the researcher could not be able to acquire students' scores in previous English literature comprehension text as a baseline measurement, as they have never had an English literature course before. On the other hand, it was also unfeasible to assess participants' literature comprehension of particular British literary works prior to providing them lessons for a period of time. This is because the content of test design was based on the specific literary works they had studied. Without teaching and learning, there would not be sufficient questions with which a literature 
comprehension test can be formed. In that assumed situation, students might also get confused about the purpose of the test itself which can in the worst-case result in a corruption of the measurements in the other two tests. Nevertheless, the first literature test was administered after one month of instruction where students in the three groups were taught differently. This introduced some system error of the applicability of the model.

A last limitation is the sample selection where all participants were from the same university in Anhui Province, China. Whether the participants reflected the average population can be questionable. The results of study may only reflect the effect of using a cultural criticism method on the cultural understanding and literature comprehension for English majors in this program and in programs that bear similar features in curriculum and instruction. See Appendix A for curriculum description of English program at AAU and Appendix B for TEM4 passing rate in the past years for English majors in this program. If the research could be implemented into more diverse teaching settings, for instance, advanced English majors in different grade level, English programs that were from different areas or from different stages of development, the research findings might be generalized to a larger population.

\section{Recommendation for Future Research and Teaching Practice}

This study applied various statistical models to examine the effectiveness of a cultural criticism approach for Chinese English majors on their cultural understanding of literary works and their literature comprehension. There are several recommendations for future research and teaching practice. 


\section{Recommendations for Future Research}

Beneficiaries of cultural criticism on literature education. The participants in the study were already at advanced level of English language proficiency. This can be seen from the fact that around $86 \%$ of them have already passed the TEM4, a standardized English exam that examined students' integrative English language ability. Therefore, a cultural criticism approach could be applicable in the classroom settings of cross-cultural literature teaching and learning for those advanced English language learners.

Yet the researcher has not paid attention to the group of students who are at the beginning and intermediate level of English language proficiency. Future research could incorporate samples that were representative of students at different levels of English language proficiency respectively and further examine the effect of cultural criticism on this population.

In addition, it would be interesting to see whether cultural criticism can be applied for literature teaching and learning beyond the restrictions of time and space. For example, native English speaking learners in contemporary society studied Old English literature or English literature in the Renaissance Times. With the passage of time, as all cultures are inherently predisposed to change, it might be beneficial for literature learners to use a cultural criticism approach to study literary works at different times.

The application of cultural criticism in the other fields. The pedagogy of cultural criticism is proven to provide support for cross-cultural literature learners to bridge their cognitive difficulties. It is important to notice that the fundamental cause of the difficulties is the conflict between the cultural referents within the original literary 
texts and readers' responses from their own cultural schemata. Furthermore, the conflict does not only exist in the literature education domain, but also in other areas including almost all cross-cultural communications. As more cross-cultural exchanges arise across the nation with the development of globalization, more cross-cultural communication is demanded for people from different background in various fields. For example, using cultural criticism in the foreign language education, in the music education as well as in the social science education, etc.

This research was also interested in the comparison of the effect between students taught by a cultural criticism approach first (group 1) and students taught by a cultural criticism approach second (group 2). Seen from the discussion of the results, group 2 that was taught by a cultural criticism approach second performed higher scores in the posttest of cultural understanding of literary works as well as in the section of cultural and historical contexts for the literature comprehension than group 1 that was taught by the cultural criticism approach second. Another possible improvement is the inclusion of some relevant learning theories, that might help explain how the instructor's proficiency levels in using a cultural criticism approach influence students' performance in cultural understanding of literary texts and literature comprehension. In addition, further research could delve into the reasons that might cause this difference. The researcher could further analyze the different focus of using a cultural criticism approach when the instructor is at different proficiency levels, and provide implications for teachers who want to be more prepared in implementing cultural criticism in their classes. Moreover, if a researcher is more interested in the effect of the instructor's proficiency levels of using a cultural criticism approach on students' cultural understanding of literary texts and literature 
comprehension, the instructor's proficiency levels could become an independent variable in the future research.

The current research also found that there was no significant difference between cultural criticism approach and traditional method on students' performance in the aspects of gender and economic status for cultural understanding of literary works as well as in the section of identification of purpose, themes and plot developments for literature comprehension. Future research could do a follow-up study for the effect of cultural criticism on these aspects. If the future study still yields the same result, the result of the current study could be further validated. Those directions are of particular interest for practitioners to be able to distinguish the two approaches.

In addition, for the current study, the researcher did not choose published validity and reliability estimates for the instruments in assessing participants' cultural understanding and literature comprehension. Future research could consider use the psychometrics of the data gathering instruments to enhance the overall quality of the instrument design.

\section{Recommendations for Teaching Practice}

The current study developed a pedagogy of cultural criticism in cross-cultural literature teaching and learning. The research provided support on the effect of utilizing a cultural criticism approach in teaching literature for cross-cultural literature learners. The teaching philosophy through a cultural criticism lens in this study for Chinese undergraduate English majors to learn British literature may serve as a reference for further implementation of cultural criticism in classroom settings focusing on crosscultural literature education. 
For the cross-cultural literature teaching practices, teachers should consider the important role of culture in helping students understand literary texts. Teachers should provide students with a point of entry into a culture other than their own (Yu, 2005), and try to bridge the cultural gaps between the students and the texts. Specifically, the following approaches based on cultural criticism method could be used for teachers to facilitate cultural understandings of literature texts: (a) consider students' cultural background; (b) provide students with opportunities to observe and experience characters' lives in the field or provide access to the first-hand resources that could reflect the culture referents within the text; (c) orient students to exploring a particular cultural issue or theme after a close reading of one or more literary works; and (d) support students' inquiry towards a particular culture and facilitate cross-cultural communication in the learning process. In literature class, students and teachers could jointly engage in cultural criticism about and questioning the cultural conflict or cultural ambiguity within the text. (e) transform the class discussion into a shared dialogue using cultural criticism. A shared dialogue is an interactive relationship with an exchange of ideas, feelings and responses in the learning process (Spears-Bunton, 2009). A shared dialogue contributes to the presentation of diverse perspectives in the learning process (Spears-Bunton, 2009). Teachers could encourage students to reflect upon the culture and people's behavior within the text through being engaged in cultural criticism.

\section{Conclusion}

The study utilized different literature teaching methods to teach British literature among Chinese undergraduate English majors in English classes at a Chinese university. By employing different methods in the teaching of a British literature course, the current 
study investigated (a) whether a cultural criticism teaching method results in better cultural understanding of literary texts in comparison with a traditional formalist and biographical teaching method; (b) whether a cultural criticism teaching method results in better literature comprehension in comparison with a traditional formalist and biographical teaching method.

This study has provided support that cultural criticism is a valuable approach to help Chinese undergraduate English majors bridge cultural gaps in their understanding of literature and facilitate literature comprehension. Therefore, instruction using a cultural criticism approach has the potential of enhancing the literary learning for cross-cultural literature learners. As a viable tool in cross-cultural literature teaching and learning, a cultural criticism approach could move students from analyzing literature from the perspectives of the author, historical context and literary mechanics to their active engagement in literature appreciation. The use of this approach in the domain of literature teaching and learning can help students bridge gaps between cultural referents within the text and the pre-existed knowledge from their cultural schemata. In other words, using cultural criticism methods in cross-cultural literature teaching and learning has a prospect in promoting cross-cultural communication and exchanges. 


\section{REFERENCES}

Akyel, A. \& Yalcin, E. (1990). Literature in the EFL class: A study of goal achievement in congruence. ELT Journal, 44(3),174-180.

Anderson, R.C., Reynolds, R.E., Steffensen, M.S., \& Taylor, M.A. (1982). Cultural schemata and reading comprehension. Reading Research Quarterly, 353-366.

Banks, J.A. (1996). The canon debate, knowledge construction, and multicultural education. In J.A. Banks (Ed.), Multicultural education: Transformative knowledge \& action (pp. 11-12). New York, NY: Teachers College Press.

Barrera, R.B. (1992). The cultural gap in literature-based literacy education. Education and Urban Society, 24(2), 227-243.

Bartlett, C (1932). Remembering. Cambridge, MA: The University Press.

Beach, R. (1997). Students' resistance to engagement with multicultural literature. In T. Rogers \& A.O. Joter (Eds.), Reading across cultures: Teaching literature in a diverse society (pp. 69-94). New York, NY: Teacher College Press.

Berger, A. (1995). Cultural criticism: A primer of key concepts. Thousand Oaks, CA: Sage.

Bosnak, M. (2009). Approach to teach English literature in the language classroom. Retrieved from http://bosnakademi.blogspot.com/2009/11/approaches-toteaching-english.html

Carrel, P.L. (1981). Culture-specific schemata in L2 comprehension. In Selected papers from the ninth Illinois TESOL/BE annual convention, First Midwest TESOL Conference (pp. 123-132). Chicago, IL: TESOL/BE.

Carter, R. \& Long, M.N. (1990). Testing literature in EFL classes: Tradition and innovation. ELT Journal, 44(3):215-221.

Caulcutt, R. (1991). Statistics in research and development. Boca Raton, FL: CRC Press.

Choo, S.S. (2011). On literature's use(ful/less)ness: Reconceptualizing the literature curriculum in the age of globalization. Journal of Curriculum Studies, 43(1), 47 67.

Cook,G. (1994). Discourse and literature. London, England: Oxford University Press.

Crystal, D. (2008). Two thousand million? English Today,24(1), 3-6. doi: $10.1017 / \mathrm{S} 0266078408000023$ 
Damen, L. (1986). Culture learning: the fifth dimension in language classroom. Reading, MA: Addison-Wesley Publishing.

Dolby, N. (2000). Changing selves: Multicultural education and the challenge of new identities. Teachers College Record, 102(5), 898-912.

Fairchild, H.P. (Ed). (1967). Dictionary of sociology and related terms. Totowa, NJ: Littlefield, Adams.

Fickel, L.H. (2009). “Unbanking” education: Exploring constructs of knowledge. In L. Spears-Bunton \& R. Powell (Eds.), Toward a literacy of promise: Joining the African-American struggle (pp.41-52). New York, NY: Routledge.

Fiedler, F.E., Terence, M, \& Harry, C.T. (1971). The culture assimilator: an approach to cross cultural training. Journal of Applied Psychology, 55(2), 95-102.

Fish, S. (1982). Is There a Text in This Class? The Authority of Interpretive Communities. Boston, MA: Harvard University Press.

Gatbonton, E. C. \& Tucker, G.R. (1971). Cultural orientation and the study of foreign literature. TESOL Quarterly. 17(2), 256-280.

Gay, G. (2000). Culturally responsive teaching: Theory, practice research, and practice. New York, NY: Teachers College Press, Columbia University.

Goodenough, W.(1964). Cultural anthropology and linguistics. In D. Hymes (Ed.), Report of the annual round table meeting on linguistic and language study (pp. 318). Washington, DC: Georgetown University Press.

Goodman, K. (1967). Reading: A psycholinguistic guessing game. Journal of the Reading Specialist, 6(4), 126-135.

Green, S. B., \& Salkind, N. J. (2007). Using SPSS for Windows and Macintosh: Analyzing and understanding data. Upper Saddle River, NJ: Prentice Hall Press.

Grossman, P. L. (2001). The teaching of second languages: Research trends. In V. Richardson (Ed.), The handbook of research on teaching (4 th ed.., pp. $416-$ 432). Washington, DC: American Educational Research Association.

Gu, L. (2001). Teaching modern Chinese literature to Non-Chinese Western readers: A critical review of Nobel Prize winner Gao Xingjian's novel. (Doctoral Dissertation). Available from Proquest Dissertations and Theses Database. (UMI No. 3123108)

Gunn, G. (1987). The culture of criticism and the criticism of culture. New York, NY: Oxford. 
Hass, G. (1978). Curriculum planning: A new approach. Boston, MA: Allyn \&Bacon.

Hebert, Y. (2001). Identity, diversity and education: A critical review of the literature. Canadian Ethnic Studies Journal, 33(3), 155-187.

Hines, M.B. (1997). Multiplicity and difference in literary inquiry: towards a conceptual framework for reader-centered cultural criticism. In T. Rogers \& A.O. Joter (Eds.), Reading across cultures:Teaching literature in a diverse society (pp.116134). New York, NY: Teachers College Press.

Hinkle, D. E., Wiersma, W., \& Jurs, S. G. (2003). Applied statistics for the behavioral sciences. Boston, MA: Houghton Mifflin Company.

Hodges, R.E. (1980). The English program, Grade Six through Nine: A heritage model. In B.J. Mandel (Ed.), Three language-Arts Curriculum Models (pp.78-90). Urbana, IL: National Council of Teachers of English.

Hu,G.W. (2004). English language education in China: Policies, progress, and reforms, Language policies, 5(4), 5-24.

Jordan, S. \& Purves, A.C. (1993). Issues in the responses of students to culturally diverse texts: A preliminary study. In L.G. Cruz (Ed.), Beyond the culture tours: Studies in teaching and learning with culturally diverse text (pp.1-22). Hillsadale, NJ: L. Erlbaum associates.

Kentner, M.A. (2005). Successful teachers of Spanish who commit to the teaching of cultures: Two qualitative case studies (Doctoral dissertation). Available from ProQuest Dissertations and Theses databases. (UMI No. 3161107)

Kim, E.H. (1982). Asian American Literature: An introduction to the writings and their social context. Philadelphia, PA: Temple University Press.

Lapp, D., Flood, J., Jensen, J. \& Squire, J. R. (Eds.). (2002). Handbook of Research on Teaching English Language Arts (2nd ed.). New York, NY: Routledge.

Li, M.S. (1998). English literature teaching in China: Flowers and thorns. The Weaver: A Forum for New Ideas in Education, 2(2), 1-17.

Lin, H.L. (1994). Toward a pedagogy for teaching feminist literature (Doctoral dissertation). Available from ProQuest Dissertations and Theses databases. (UMI No. 3436722)

Liu, W. (2013, June). Innovative Teaching on the Course of American Literature: Some practices in SXRTVU. In 2013 Conference on Education Technology and Management Science (ICETMS 2013). Atlantis Press. 
Malcolm, I.G., \& Sharifian, F. (2002). Aspects of aboriginal English oral discourse: An application of cultural schema theory. Discourse Studies, 4(2), 169-181.

Milner, D. (1983). Children and race. Beverly Hills, CA: Sage.

Moody, H.L.B. (1968). The Teaching of Literature. London, England: Longman.

Moran, P.R. (2001). Teaching culture: Perspectives in practice. Boston, MA: Heinle \&Heinle.

Peregoy, S. F., \& Boyle, O. F. (2000). English learners reading English: What we know, what we need to know. Theory into practice, 39(4), 237-247.

Philipsen, G. (1987). The prospect of cultural communication. In D. Kinkaid (ed.), Communication theory from eastern and western perspectives (pp. 12-20). New York, NY: Academic Press.

Pritchard, R. (1990). The effects of cultural schemata on reading processing strategies. Reading Research Quarterly, 25(4), 273-295.

Robinson, G. (1985). Cross-cultural understanding. New York, NY: Noble and Noble.

Robbins, L.S., Fantone, J.C., Hermann, J., Alexander, G.L., \& Zwerfler, A.J. (1998). Culture, communication, and the informal curriculum: Improving cultural awareness and sensitivity training in medical school. Academic Medicine, 73(10), S31-34.

Rogers, T. (1997). No imagined peaceful place: a story of community, texts, and cultural conversation in one urban high school English classrooms. In T. Rogers \& A.O. Soter (Eds.), Reading across cultures:Teaching literature in a diverse society (pp.69-94). New York, NY: Teacher College Press.

Rosenblatt, L. M. (1968). Literature as Exploration. New York, NY: Noble and Noble.

Rojas-Rimachi, L.M. (2011). Teaching culture through language and literature: the intersection of language ideology and aesthetic judgement (Doctoral dissertation). Retrieved from ProQuest Dissertation and Theses Database. (UMI No. 4323125)

Rumelhart, D. E. (1980). Schemata: The building blocks of cognition. In R. J. Spiro, B.C. Bruce \& W.F. Brewer (Eds.), Theoretical issues in reading comprehension (pp. 13-20). Hillsdale, NJ: Lawrence Erlbaum.

Song,Y. (2009). How can Chinese English teachers meet the challenge of creating a learner-centered, communicative, intercultural classroom to achieve optimal student learning outcomes? Canadian Social Science, 5(6), 81-91. 
Soter, A.O. (1997). Reading literature of other cultures: some issues in critical interpretation. In Rogers, T. \& Soter, A.O. (Eds.) Reading across culture:

Teaching literature in a diverse society. (pp. 213-219). New York, NY: Teachers College Press.

Spears-Bunton, L.(1992).Cultural consciousness and response to literary texts among African-American and European-American high school juniors. Lexington, KY: University of Kentucky.

Spears-Bunton, L. (2009). Along the road to social justice: A literacy of promise. In L. Spears-Bunton \& R. Powell (Eds.), Toward a literacy of promise: Joining the African-American struggle (pp. 23-39). New York, NY: Routledge.

Steffensen, M.S., Joag-deve, C \& Anderson, R.C. (1979). A cross-cultural perspective on reading comprehension. Reading Research Quarterly, 15, 10-29.

Suliman. (1980). The reader and the text. Princeton, NJ: Princeton University Press.

Tapp, S.D. (2007). Cultural competence education: A user-friendly instrument to assess professional development needs. In M.A. Trent, T. Grizzle, M. Sehorn, A. Lang, \& E. Rogers (Eds.), Religion, culture, curriculum, and diversity in $21^{\text {ST }}$ century America (pp. 29-39). Lanham, MD: University Press of America.

Tchudi, S. (1991). Planning and assessing the curriculum in English Language Arts. Alexandria, VA: The Association for Supervision and Curriculum Development.

Tchudi, S.J. \& Tchudi, N.T. (1999). The English Language Arts handbook: Classroom strategies for teachers. Portsmouth, NH: Boynton/Cook.

Tiedt, I.M. (1983). The language arts handbook. Englewood Cliffs, NJ: Prentice-Hall.

Temple, C., Martinez, M., \& Yokota J. (2006). Children's books in children's hands: An introduction to their literature (3rd ed.). Boston, MA: Allyn and Bacon.

Tyson, L. (2014). Critical theory today: A user-friendly guide. Florence, KT: Routledge.

Wang, P.L. (1999). Anthology of British literature and history. Shengyang, China: Northeast Normal University Press, China.

Wen, C. (2010). The impact of TEM-8 (Test for English Major Band 8) on English majors in China. Retrieved from http://diva-portal.org/smash/get/diva2:328660/ FULLTEXT01.pdf?trk=profile_certification_title

Winkelman, M. (2005). Cultural awareness, sensitivity, \& competence. Peosta, IA: Eddie Bowers Publishing. 
Yu, R.D. (2005). Taking a cultural-response approach to teaching multicultural literature. The English Journal, 94(3), 55-60.

Zhen, C. (2012). Characteristics and strategies of literature teaching in the EFL context in China. International Journal of Electronics and Communication Engineering, 5(3), 35-43. 


\section{APPENDICES}




\section{Appendix A}

\section{Curriculum Description for English Program at Anhui Agricultural University}

Basically, different curriculums have been designed and implemented to achieve the two-fold teaching objectives -- English language acquisition and comprehensive English competencies for English majors in this program at Anhui Agricultural University. Students at the early stage of the program (first-year and second-year students) mainly focus on language acquisition. The courses offered at this stage include College Comprehensive English Course, College Extensive English Reading, Listening, Speaking, Writing. Students at the advanced stage of the program (third-year and fourthyear students) need English language courses that can further enhance their ability in language acquisition, but also need the professional courses such as translation and interpreting between Chinese and English languages, English literature, English linguistics, pedagogy for English language teaching, legal English, etc. The purpose of incorporating these professional courses is to facilitate the development of students' comprehensive English competencies at the advanced level.

Among them, British literature and American literature are two required mandatory literature courses offered for all of students who are at the third-year and at the fourth-year of the program respectively. 


\section{Appendix B}

\section{Test for English Majors, Band 4 (TEM-4)}

By the third year of study majoring in English, the students in this program have already taken Tests for English Majors, Band 4 (TEM-4) and are preparing for the TEM 8. TEM is a kind of standardized exam organized by the Higher Education Institution Foreign Language Major Teaching Supervisory Committee since 1991 (Wen, 2010). TEM aims at measuring students' integrative English language competencies with an emphasis on students' ability to use English as a foreign language (Wen, 2010). Taking the TEM-4 successfully is a milestone for English majors. It signifies that they have benefited from the rigorous language testing system and have a solid foundation in the English language (Wen, 2010). According to the statistics in the year of 2015, almost $86 \%$ students at the third year in the English program of Anhui Agricultural University passed the TEM-4 (The passing score is above 60 points), and around $31 \%$ students at the third year achieved a good or excellent level when taking TEM-4 (The scores ranging from 70 to 80 were recorded as good; the scores above 80 were recorded as excellent).

\section{Reference:}

Wen, C. (2010). The impact of TEM-8 (Test for English Major Band 8) on English majors in China. Retrieved from http://diva-portal.org/smash/get/diva2:328660/ FULLTEXT01.pdf?trk=profile_certification_title 
Appendix C

\section{Fall British Literature Course Syllabus \\ Anhui Agricultural University}

\section{Instructor's Contact Information}

Instructor: Zhang Yu

Email: zhangyutop912@sina.com

Hours: Every Weds. Morning and by appointment

Office: Room 501, School of Foreign Languages.

\section{Required Course Texts (In English Version):}

《英国文学史及作品选读》王佩兰, 东北师范大学出版社, 1999.

1. Beowulf

http://www.doc88.com/p-6778129492905.html

2. Hamlet

http://www.doc88.com/p-703036565.html

3. Paradise Lost

http://www.doc88.com/p-0728015350200.html

4. Gulliver's Travel (Download here)

http://www.en8848.com.cn/e/DownSys/DownSoft/?classid=22\&id=4014\&pathid=0

5. $\quad$ Pride and Prejudice

http://www.doc88.com/p-808215660393.html

6. $\quad$ Oliver Twist (Download here)

http://www.en8848.com.cn/e/DownSys/DownSoft/?classid=22\&id=65\&pathid=0

7. Mrs. Dalloway (Download here)

http://www.en8848.com.cn/e/DownSys/DownSoft/?classid=22\&id=4197\&pathid=0 


\section{References:}

Daems, J (2006). Seventeenth-Century Literature and Culture. London, LD: Continuum Books.

Hopkins, L \& Steggle, M (2006). Renaissance Literature and Culture. London, LD: Continuum Books.

Ruston, S.(2006). Romanticism. London, LD: Continuum Books.

Moran, M (2006). Victorian Literature and Culture. London, LD: Continuum Books

Gunn, G. (1987). The culture of criticism and the criticism of culture. New York, NY:Oxford.

\section{Course Description}

This course centers on an examination of British literature and culture from the period of Middle Ages to the Modernism and Post-modernism. The following literary periods will be introduced during the course: the Middle Ages, the English Renaissance, the period of Revolution and Restoration, the Age of Enlightenment, the Romantic Period, The Victorian Age, the Modernism and Post-Modernism. In each literary period, you will read one representative literary work per the course requirement, that includes: Beowulf, Hamlet, Paradise Lost, Gulliver's Travel, Pride and Prejudice, Oliver Twist, and Mrs. Dalloway. The emphasis in the course will be on analyzing and interpreting literary texts using a cultural criticism approach. Cultural criticism examines the elements of culture and how they affect one's perceptions and understanding of texts. Throughout the course, you will not only know the diverse cultural knowledge in the British society, but also learn how to engage in cross-culture practice using a cultural criticism approach. You will make connections between the texts you have read and the cultural contexts such as the historical, economic, and political contexts in which those texts emerged. It will help you move beyond the boundaries of the text itself to establish links among texts, values, institutions, groups, practices, and people. By the end of the semester you should be able to analyze specific texts closely in relation to relevant historical/cultural contexts, and synthesize the themes of the various literary periods of this era using a cultural criticism approach.

\section{Cultural Criticism Units of Instruction}

Cultural criticism (Gunn, 1987) is one of critical lens through which any text can be viewed. It focuses on the elements of culture and how they affect one's perceptions and understanding of texts. This form of criticism examines how different religions, ethnicities, class identifications, political beliefs, and views affect the ways in which texts are created and interpreted (Gunn, 1987). Cultural criticism suggests that being a part of- 
or excluded from-a specific group or culture contributes to and affects our understanding of texts.

In our British literature class, each class session consists of students' presentations, discussions, the instructor's feedback and assignments. In each teaching procedure, it will follow the steps of using cultural criticism in the flow chart below (developed by Zhang $\mathrm{Yu})$

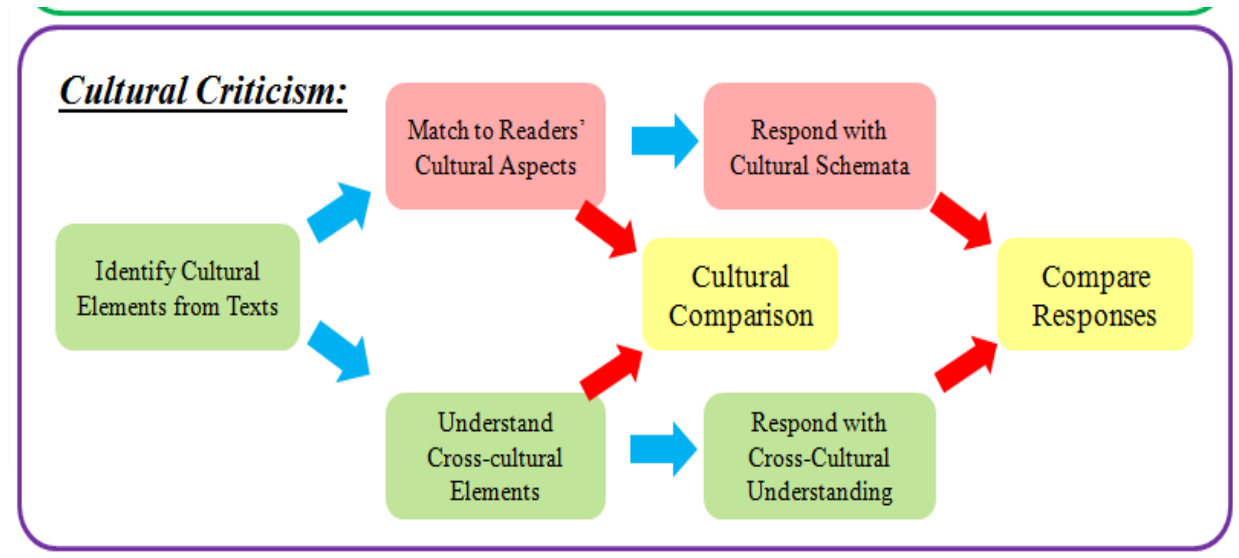

Specific directions will be provided in the class.

\section{Course Goals and Guiding Questions}

The primary aim of this course is to help you develop ways to think about, study, research, discuss, and write about the diversity of British literature and culture from the Middle Ages to the Modernism and Post-modernism. You might also think of British Literature and Culture as a course in how to read primary documents and to use those documents to construct a literary and cultural history of "British" (or what would become the British from the Middle Ages to the Modernism and Post-modernism.

Because the study of literature and culture is interdisciplinary, the range of issues we will explore is quite varied at times. Don't be scared or annoyed by this; be fearless and excited about it. Our primary purpose will always be to invent ways of analyzing, thinking about, talking about, and writing about the connection between literature and culture in the period.

\section{A set of guiding questions should help us to get started and to remain focused.}

- What are the connections between writing and culture in this era?

- How do we read and interpret these texts from the past?

- What do they tell us about Britain and British cultures in the literary period? In what ways do our reading strategies limit or enhance what the texts disclose?

- In what ways can these documents serve as evidence for cultural-historical claims and interpretations? 
- What did these texts do? What purposes or functions did these documents serve in their original contexts? And how do we know that they served such functions?

- What are the uses of these texts for us in the present?

\section{Class Assignments}

\begin{tabular}{llll}
\hline Chapter & Literary Period & Selected Literary Texts & Week \\
\hline 1 & The Middle Ages & Beowulf & Weeks 3-4 \\
2 & The English Renaissance & Hamlet & Weeks5-6 \\
3 & $\begin{array}{l}\text { The period of Revolution and } \\
\text { Restoration }\end{array}$ & Paradise Lost & Weeks 7-8 \\
4 & The Age of Enlightenment & Gulliver's Travel & Weeks 9-10 \\
5 & The Romantic Period & Pride and Prejudice & Weeks 10-11 \\
6 & The Victorian Age & Oliver Twist & Weeks 12-13 \\
7 & Modernism & Mrs. Dalloway & Weeks 14-15 \\
\hline
\end{tabular}

\section{Course Requirements}

\section{Major Discussion Topics in the Assigned Texts}

These will be covered in the form of lectures, discussions, research paper, oral presentations, etc. This is not an exhaustive list, but it provides an overview of the major topics/issues to be covered. Others will emerge through readings and discussion.

- tribal culture in Beowulf,

- humanism in Hamlet,

- religion perspectives in Paradise Lost,

- imperialism in Gulliver's Travel,

- marriage in Pride and Prejudice,

- $\quad$ poverty and criminality in Oliver Twist,

- feminism in Mrs. Dalloway

Reading. The most important work in this British literature course involves careful, patient, thorough reading of the assigned texts. You will need to finish the assigned readings by the time indicated on the reading schedule.

\section{Leading Discussion \& Presentation:(30\% out of final grades)}


In this course, students will be divided into several learning group. Each student group will be assigned a literary piece in a certain literary period. Each student group will take turns to lead class discussion for the assigned literary texts per week. Each student group will develop a five-page writing pieces (critical cultural incident study ) out of their assigned literary work and present it in class. Student group are encouraged to use PowerPoint presentations. Each student group will respond to other students' answers. Specific direction will be provided later in the class. DUE: AS ASSIGNED

\section{Five Essential Questions: (10\% out of final grades)}

Required by Student Group: Each student group will pose, post and answer five essential questions. The questions should connect with the social cultural influence on the preselected themes and distribute them to the class one week ahead of class time. The specific direction will be provided later in the lass.

Required by Each Student: Each Student will answer the five essential questions verbally \& in 1-2 page/slide summation. The answers to five essential questions should include 1-2 citations, an example from any kind of media, the arts, blogs, photography, web page books, etc. to support or make their point. summation will be collected in the class.

\section{Notebook:}

At least two notebooks (Three notebooks are preferred) should be prepared for class.

a) One is for class notes;

b) One is for in-class assignments.

c) You are also encouraged to keep a reading journal or taking assigned readings by the time indicated on the reading schedule. You are encouraged to keep a reading journal or take reading notes on each text. If you don't take notes of some sort, I'm not sure how you will be able to keep track of the complexity an diversity of your reading throughout the semester.

\section{Assessment:}

There are a total of four assessments in this class. Specific direction will be provided later. See the details below:

1) British Cultural Analysis Essay -Romeo and Juliet (Administrate in early Sept. 2015)

$10 \%$ out of final grade

2) British literature comprehension I (Administrate in early Sept. 2015)

$10 \%$ out of final grade

3) British Cultural Analysis Essay- Pride and Prejudice (Administrate in late Nov. 2015)

$15 \%$ out of final grade

4) British literature comprehension II (Administrate in Dec. 2015) 
$15 \%$ out of final grade

\section{Attendance (10\% out of final grade)}

In addition to careful reading, I expect active participation and good attendance from everyone. Some of the most significant learning will happen during our classroom conversations. Thus, I am firm believer in class attendance. Irregular attendance will hurt your grade; good attendance and active participation in class discussions will improve your grade. If you miss no classes, your grade will move up one step ( 85 will become an 90 , for instance). If you miss three classes, your grade will move down one step (80 could turn into 75 ). If you miss five, you will fail in this class.

You will be expected to read and analyze each piece of literature assigned in the course, as well as the background material and the assigned critical works. I would strongly suggest that you read each work, along with the introduction to the author, closely and take notes and mark important passages. You should then make connections to relevant historical and cultural contexts based on the background lectures and the critical works and background material. This kind of individual synthesis, founded on classroom discussion, will be crucial to your complete understanding of these works and the ideas connected to them and thus to your success on the exam.

\section{Grades}

Any work turned in late (ie, after the due date) may be downgraded ten points per day late.

1) British Cultural Analysis Essay -Romeo and Juliet $10 \%$

2) British literature comprehension $10 \%$

3) British Cultural Analysis Essay- Pride and Prejudice 15\%

4) British literature comprehension II $15 \%$

5) Group Leading Discussion $30 \%$

6) Five essential questions $10 \%$

7) Attendance $10 \%$

The grading scale for the class will be as follows:

90 to $100 \%=$ Excellent Work

80 up to $90 \%=$ Strong Work; Well Above Average

70 up to $80 \%=$ Average/Satisfactory Work

60 up to $70 \%=$ Below Average

Below $60 \%$

\section{Tentative Course Schedule and Assignments}

(subject to change at the instructor's discretion):

Week 1 (Aug. 24-30): An Overview of the Course, Introductory Activity:the concept of cultural criticism in literature teaching; Prepare notebooks

Assignments: Read Selected literary Scripts from Romeo and Julie 
literature

Week 2 (Aug. 31-Sep.6): Guided practice: using cultural criticism in learning

--a sample literature text: Romeo and Juliet

a discussion model

student group formed

Assignments: Read Beowulf

\section{The Middle Ages}

Week 3 (Sept. 7-13): Overview of the literary works in the Middle Ages

Reading and Analyze Beowulf

Assignments: Continue reading Beowulf

Answer five essential questions for Beowulf posted by Group 1

Week 4 (Sept. 14-20): Leading discussion \#1 Beowulf

Assignments:

--Finish reading and analyzing Beowulf

--Research related literature resources in the English Renaissance period

--Read Hamlet

\section{The English Renaissance}

Week 5 (Sept. 21-27): English Renaissance Overview

--Reading and analyze Hamlet

Assignments:

Continue reading Hamlet

Answer five essential questions for Hamlet posted by Group 2

Week 6 (Sept. 28-Oct.4): Leading discussion \#2 Hamlet

Assignments:

--Finish reading and analyzing Hamlet

--Research related literature resources in the period of Revolution and

Restoration

--Read Paradise lost

Week 7 (Oct.5-Ocb.11) National Holiday, No Class

Period of Revolution and Restoration Overview

Reading and analyze Paradise Lost

Assignments:

-- Continue reading Paradise Lost

-- Answer five essential questions for Paradise Lost posted by Group 3

\section{Week 8 (Oct.12-Oct.18): Leading discussion \#3 Paradise Lost}

Assignments:

--Finish reading and analyzing Paradise Lost

--Research related literature resources in the Age of Enlightenment

--Read Gulliver's Travel 


\section{The Age of Enlightenment}

Week 9 (Oct.19-25) The Age of Enlightenment Overview

Reading and analyze Gulliver's Travel

Assignments:

--Continue reading Gulliver's Travel

-- Answer five essential questions for Gulliver's Travel posted by Group 4

Week 10 (Oct.26-Nov.1 ): Leading discussion \#4 Gulliver's Travel Assignments:

-- Finish reading and analyzing Gulliver's Travel

--Research related literature resources in the period of Romanticism

--Read Pride and Prejudice

\section{Romanticism}

Week 11 (Nov. 2-8): Romanticism Overview

Reading and analyze Pride and Prejudice

Assignments

-- Reading Pride and Prejudice

-- Answer five essential questions for Pride and Prejudice posted by Group 5

Week 12 (Nov. 9-15 ): Leading discussion \# 5 Pride and Prejudice Assignments:

-- Finish reading and analyzing Pride and Prejudice

--Research related literature resources in the Victorian Age

--Read Oliver Twist

\section{The Victorian Age}

Week 13 (Nov.16-22 ): The Victorian Age Overview

Read and analyze Oliver Twist

Assignments:

-- Answer five essential questions for Oliver Twist posted by Group 5

-- Read Oliver Twist

Week 14 (Nov. 23-29): Leading discussion \# 6 Oliver Twist

Assignments:

-- Finish reading and analyzing Oliver Twist

--Research related literature resources in the Modernism and Post-modernism

--Read Mrs. Dalloway

\section{Modernism and Post-modernism}

Week 15 (Nov. 30-Dec. 6): The Modernism and Post-Modernism Overview Read and analyze Mrs. Dalloway

Assignments:

Answer five essential questions for Mrs. Dalloway for by Group 7

-- Read Mrs Dalloway 
Week 16 (Dec. 7-14): Final Week. Class Review

Week 16 (Dec. 7-.13): Leading Discussion \# 7 Mrs. Dalloway

Course wrap-up

Assignments:

Finish reading and analyzing Mrs. Dalloway 


\section{Appendix D}

\section{Group Leading Discussion Model for Practice Using Cultural Criticism}

Guideline: Read Romeo and Juliet thoroughly, select the scripts that are related to the following theme: Betrayal of arranged marriage in beloved people. Every group should assign three pieces of selected literature texts to the class, and make sure the group puts forward five essential questions that are connected to the social cultural influence (for example, parental authority, social status, economic security, and pedigree, class consciousness) on the marriage for the class before formal discussion.

\section{I: Selected Literary Texts in Romeo and Juliet by William Shakespeare}

\section{Romeo and Juliet Selected Scripts 1}

Act 2, Scene 2, Capulet's Garden.

Scripts could be found in the following links:

http://nfs.sparknotes.com/romeojuliet/page_78.html

\section{Romeo and Juliet Selected Scripts 2}

Act 3, Scene 5, Capulet's orchard.

Scripts could be found in the following links:

http://nfs.sparknotes.com/romeojuliet/page_186.html

\section{Romeo and Juliet Selected Scripts 3}

Act 5, Scene 3, A churchyard; in it a tomb belonging to the Capulets

Scripts could be found in the following links:

http://nfs.sparknotes.com/romeojuliet/page_260.html 


\section{III: Sample Group Leading Questions:}

To answer the following questions, you may contextualize the British social culture in $16^{\text {th }}$ century by researching the relevant literature to support your viewpoints.

1) What is Juliet's dad, Lord Capulet's attitude towards his daughter's marriage? How would you characterize the Capulets' marriage? Why does he hold such an attitude? Please justify your answer based on the British social culture in $16^{\text {th }}$ Century.

2) Why do Romeo and Juliet marry in secret? What are the consequences of such secrecy?

3) How does the social culture (i.e. parental authority, relationship between two families, class consciousness) influences on the choice of marriage for the characters in the texts? Please choose at least one character in the novel to answer this question.

4) What are Romeo and Juliet's attitudes/values towards marriage? How does it conflict with their parents? What might account for the different cultural values towards marriage between Romeo and Juliet and Juliet's parents?

\section{Choose one of the following questions:}

1) What were the people's attitude towards marriage in the $16^{\text {th }}$ century in China? Compare it with the values on marriage in Romeo and Juliet's story.

2) Romeo and Juliet sacrificed their lives in opposition to the marriage that Juliet's family wanted her to have. In your opinion, could Romeo and Juliet be considered a respectable couple in the modern Chinese society? Why? Please justify your answer.

\section{II: Cultural Incident Study}

\section{Directions:}

In Romeo and Juliet, Marriage is seemingly always on the minds of the characters in Romeo and Juliet. As we see in the novel, Juliet's attitude towards marriage always conflicts with her parents' views. For Juliet, marriage is a way of formally recognizing a shared emotional bond (love). For her parents, marriage is a means of securing wealth, status, and stability. Find out a literary script Romeo and Juliet that addresses the same 
theme. Keeping your attention focused on the theme of marriage and the elements of culture related to the marriage (for example, parental authority, social status, economic security, and pedigree, class consciousness). Then depict the cultural influences on the choice of marriage for the characters Romeo and Juliet, analyze the possible causes of the young woman, Juliet's betrayal of arranged marriage from her family. In this study, you need to contextualize the British social culture for marriage in the $16^{\text {th }}$ Century. You will need use a primary or secondary sources to help you secure you own grasp on the main points of values towards marriage in Britain. The paper should be a five-page narrative writing piece. 
Appendix E

KWHL Chart for Practice

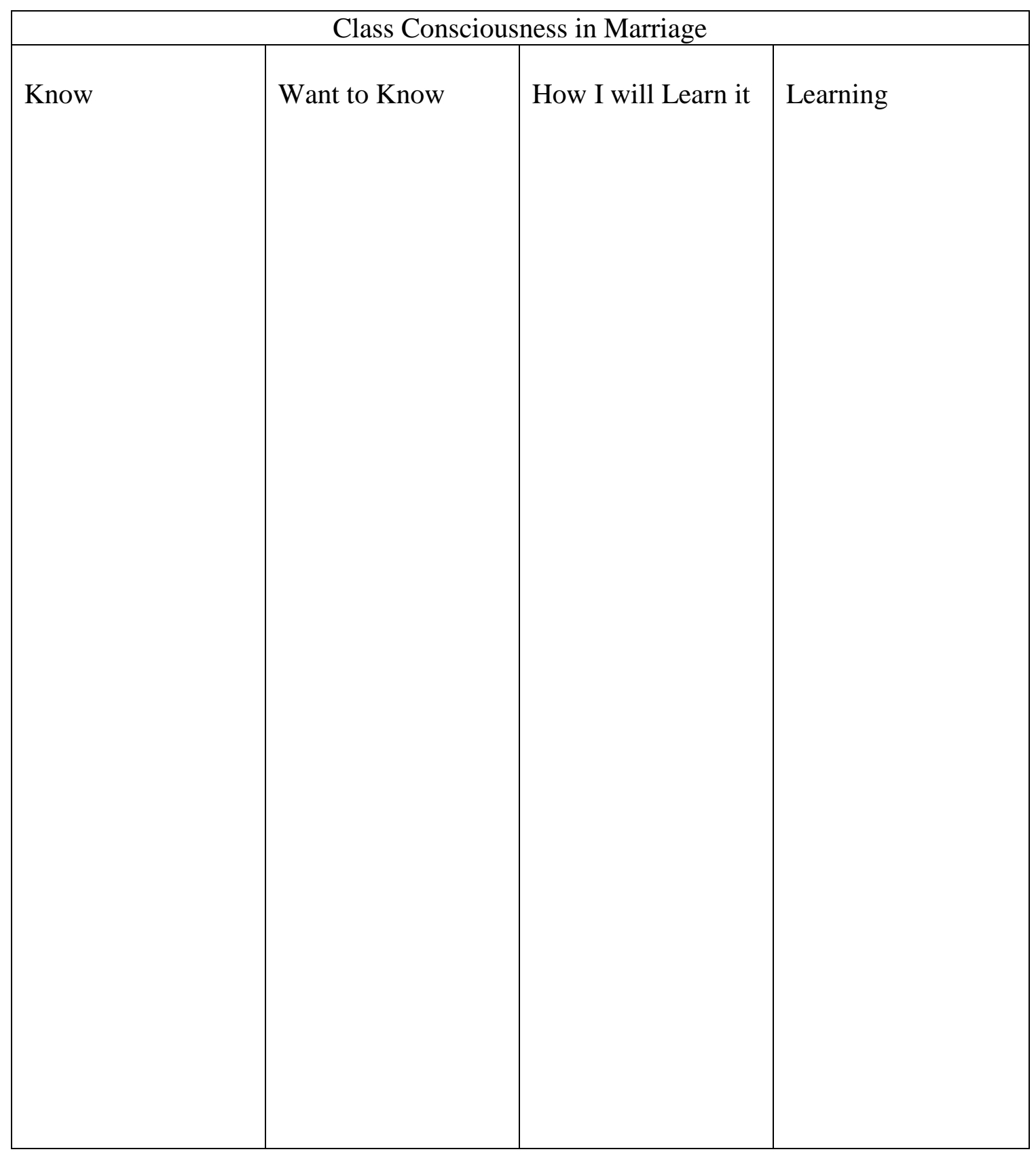


Appendix F

\section{Cultural-Response Assignment}

\section{Tracing a Cultural Criticism Perspective:}

In this assignment, you are required to list the element of culture in the assigned reading. Please include the following aspects in your response.

Title of Text:

Author:

Genre:

List the element of culture you have found in the reading:

The location of the text:

Element of Culture:

Is this element of cultural familiar, alien to you? 
List elements that are close to cultural elements in your own culture. How can you connect them with your own culture?

List elements that are different from cultural elements in your own culture. How can you differentiate them from your own culture?

How does the element of culture help you understand the text? 


\section{Appendix G}

\section{A Teaching Model for Romeo and Juliet Using Traditional Formalist and}

\section{Biographical Method}

\section{The Renaissance (1500-1600)}

A period of the breaking up of feudal relations and the establishing of the foundations of capitalism, marked by a flourishing of national culture. In the period, the classical works were translated into English and studied by the humanists, who held their chief interest in man, his environment and doings and fought for the emancipation of man from the tyranny of the church and the religious dogmas.

\section{Social Background}

James I: "Divine-right" theory

King was God's Lieutenant on earth

Parliament and people had no right to question the king's action

$\star$ civil war in 1641

New-born capitalist class: Puritans and the Parliament Influence:

(a) abolish monarchy

b) England was declared a Commonwealth

\section{William Shakespeare (1564-1616)}

William Shakespeare: the greatest English dramatist and poet, one of the 
first founders of realism. He produced 37 plays, two narrative poems and 154 sonnets. His first period as a dramatist includes his earliest plays, two of his well-known early tragedies (Romeo and Juliet" and "Julius Caesar") and all his romantic comedies. The second period includes all his great tragedies ("Hamlet", “Othello", "King Lear", “ Macbeth”) and some of his earlier tragic-comedies. The third period includes chiefly his three last tragicomedies. The themes in his dramas that reflect truthfully the social contradictions of his age are preserved with power chiefly through the vivid portraits and elaborate psychological analysis of his dramatic characters. He used the English language with the greatest freedom and ease, so that almost all the speeches fit all the characters.

\section{Four Period of Dramatic Career}

- 1) Apprenticeship:

$>$ History plays

$>$ Comedies

- 2) Individualized:

History plays

$>$ Comedies

$>$ Tragedies (Romeo and Juliet)

- 3) Great tragedies

$>$ Four tragedies: Hamlet, Othello, , King Lear, Macbeth

$>$ Dark comedies

- 4) Romantic tragicomedies 


\section{Shakespeare's Achievements}

- Represented the trend of history in giving voice to the desires and aspirations of the people.

- Stage 1-2: plays written contextualized the history of England

- Reflect the humanism:

- Early stage, plays are full of optimistic spirit

$>$ firm belief in the nobility of human nature

$>$ in the power of love.

- Stage 2-3, plays reflect the ugliness of human nature.

- $\quad$ Stage 4 , beautiful romances

$>$ recovered his faith in human nature

- "Round" character from all walks of life

$>$ ranging from kings to clowns, and grave-diggers; from lunatics to ghosts; from lovers to man-haters.

$>$ Character have many aspects in characteristics

$\star$ play construction

$\star$ great poetry

^ sophisticated language

Romeo and Juliet Teaching Model

Introduction of The Play Romeo and Juliet's Plot:

There are two families in Italy who share mutual hostility to each other. One is Montague; the other is Capulet. One day Romeo Montague participated in a fancy dress party held by Capulet home, where he met Juliet Capulet and they fell in love with each other at first sight. 
Romeo and Juliet secretly got married with the help of the priest, who hopes to eliminate the long-standing hatred between the two families. Later, Romeo killed Juliet's cousin and was punished by expulsion

After hearing the bad news, Juliet comes to the Father for help, who helps her drink a poison to pretend committing suicide but can wake up 42 hours later. Romeo arrives at the church in a hurry. Believing Juliet has already died he drinks a toxic drug and kills himself. When Juliet wakes up, she also chooses to take her own life. In the end, the younger generation's love tragedy leads to the reconciliation between two families.

\section{Read Romeo and Juliet Act II Scene II, and then answer the following}

\section{questions:}

1) Try to summarize the main idea of each paragraph. What is the theme of this Act?

2) What does Romeo wish for, as he watches Juliet lean her cheek on her hand?

3) How does Romeo comment on Juliet? What kind of feeling Romeo has when he spoke with Juliet? To what does Romeo compare Juliet's beauty?

4) What does this quote mean "oh speak again, bright angel! For thou art as glorious to this night, being o'er my head as is a winged messenger of heaven?"

5) Why does Juliet persistently persuade Romeo to deny his father and refuse his name?

\section{Selected Literary Scripts from Act II, Scene II}

ROMEO

But, soft! what light through yonder window breaks?

It is the east, and Juliet is the sun.

Arise, fair sun, and kill the envious moon,

Who is already sick and pale with grief,

That thou her maid art far more fair than she: 
Be not her maid, since she is envious;

Her vestal livery is but sick and green

And none but fools do wear it; cast it off.

It is my lady, O, it is my love!

O, that she knew she were!

She speaks yet she says nothing: what of that?

Her eye discourses; I will answer it.

I am too bold, 'tis not to me she speaks:

Two of the fairest stars in all the heaven,

Having some business, do entreat her eyes

To twinkle in their spheres till they return.

What if her eyes were there, they in her head?

The brightness of her cheek would shame those stars, As daylight doth a lamp; her eyes in heaven

Would through the airy region stream so bright

That birds would sing and think it were not night.

See, how she leans her cheek upon her hand!

$\mathrm{O}$, that I were a glove upon that hand,

That I might touch that cheek!

JULIET

Ay me!

ROMEO

She speaks: 
O, speak again, bright angel! for thou art

As glorious to this night, being o'er my head

As is a winged messenger of heaven

Unto the white-upturned wondering eyes

Of mortals that fall back to gaze on him

When he bestrides the lazy-pacing clouds

And sails upon the bosom of the air.

\section{JULIET}

O Romeo, Romeo! wherefore art thou Romeo?

Deny thy father and refuse thy name;

Or, if thou wilt not, be but sworn my love, And I'll no longer be a Capulet.

ROMEO

[Aside] Shall I hear more, or shall I speak at this?

\section{JULIET}

'Tis but thy name that is my enemy;

Thou art thyself, though not a Montague.

What's Montague? it is nor hand, nor foot,

Nor arm, nor face, nor any other part

Belonging to a man. $\mathrm{O}$, be some other name!

What's in a name? that which we call a rose

By any other name would smell as sweet;

So Romeo would, were he not Romeo call'd, 
Retain that dear perfection which he owes Without that title. Romeo, doff thy name, And for that name which is no part of thee Take all myself. 
Appendix $\mathrm{H}$

\section{Checklist for Validation of Cultural Criticism Units of Instruction}

\section{Observer's Name:}

\section{Class Visited:}

\section{Date and Time:}

\section{Directions for Using this checklist:}

Please place a Yes at the end of each entry if you think the given lesson includes the following procedures;

Please place a No at the end of each entry if you think the given lesson does not include the following procedures.

- Students' prior cultural knowledge was activated in general

- Students were taught with culture-related knowledge within the literary text

- Students were invited to respond for the culture-related features of the text

- Students were guided to share with their perspectives of cultural understanding within the learning community

- Students were guided to connect their own cultural analysis to analysis of British cultural values in the literary texts

- Students were guided to bridge the gap between cultural referents within the original literary texts and readers' responses based on their own cultural schemata

\section{Additional Comments:}




\section{Checklist for Validation of Traditional Formalist and Biographical Units of}

\section{Instruction}

\section{Observer's Name:}

Class Visited:

Date and Time:

\section{Directions for Using this checklist}

Please place a Yes at the end of each entry if you think the given lesson includes the following procedures;

Please place a No at the end of each entry if you think the given lesson does not include the following procedures.

- Instructor provides an overview of a particular literature period

- Instructor presents the life of times of the authors

- Instructor asks students summarizing the main ideas of literary works

- Instructor assists students in analyzing plot developments

- Instructor ensures students have a close reading of selected literary text

\section{Additional Comments:}




\section{Appendix I}

\section{The Cultural Analysis of Britain in Romeo and Juliet}

(Used as a Pre-test for testing student's cultural understanding of literary texts)

\section{Introduction to the Assignment:}

This assignment asks you to write a critical essay that includes the cultural analysis of a literature text: Romeo and Juliet. This assignment is built on the premise that understanding a particular culture will help us better appreciate and comprehend that culture's literary productions, just as a careful reading of a literary text may lead us to better understand the culture from which it emerged.

For the purposes of this assignment, the definition of "cultural analysis" is large and open-ended. It means making connections between the texts we've read and the cultural contexts in which those texts emerged; it means making connections between texts and the cultural contexts in which those texts circulate. It does not exclude the formal or internal analysis of a text (indeed some of the very best cultural criticism uses formal analysis of literary texts); but cultural analysis moves beyond the boundaries of the text itself to establish links among texts, values, institutions, groups, practices, and people.

\section{Specific Directions:}

In Romeo and Juliet, Marriage is seemingly always on the minds of the characters in Romeo and Juliet. As we see in the novel, Juliet's attitude towards marriage always conflicts with her parents' views. For Juliet, marriage is a ways of formally recognizing a shared emotional bond (love). For her parents, marriage is a means of securing wealth, status, and stability. Read through Romeo and Juliet. Keeping your attention focused on the theme of marriage and the elements of culture related to the marriage (for example, parental authority, social status, economic security, and pedigree, class consciousness). Then depict the cultural influences on the choice of marriage for the characters Romeo and Juliet, analyze the possible causes of the young woman, Juliet's betrayal of arranged marriage from her family. In this study, you need to contextualize the British social culture for marriage in the 16th Century. You will need use a primary or secondary sources to help you secure you own grasp on the main points of values towards marriage in Britain.

The specific form and content of your cultural analysis paper should include the following aspects: (a) customs and beliefs towards marriage (b) gender roles concerning marriage; (c) economic status concerning marriage; (d) politics concerning marriage; (e) free will concerning marriage.

Your papers may be deeply informed by a theory or may not. Some will want to develop a very precise idea of what "cultural analysis" means; others will not. Some will use a 
great deal of historical research; others only a little. Some papers will use mostly primary documents to construct an understanding of a British cultural context; others will rely on secondary sources; and others may use a mix of both. All these papers, however, must use documents and sources beyond the literary text itself. When you cite others' sources, don't forget to list where you cite them \& create a reference at the end of paper.

What-I'm-Really-Looking-For. Just so you know, when I'm reading these papers, I'll be asking myself the following questions:

- Does the paper move beyond a formal analysis of a text in isolation?

- Does it focus on the literature and culture of Britain in the Shakespeare's'Time?

- Does it make links between texts and values, institutions, groups, practices, or people?

- Does the paper make specific and interesting claims about the text(s) and culture(s) being examined?

- Does it explain in a clear and persuasive manner its interpretation of those texts and contexts?

- Does it support that interpretation with judiciously chosen evidence?

- Is it organized in a way that makes clear (rather than detracts from) the argument's major claims and emphases?

- Does it acknowledge its primary and secondary sources using a bibliography and a clear and consistent style of documentation?

Requirements: The paper should be at least 5 pages writing pieces. Time New Roman 12 Font. Double Spaces. Only Print-Out Version Accepted.

It is due on Weds., September 3, 2015. Bring the paper copy to class \& also save the digital version in your computer.

The Assignment Evaluation: Please make sure you read the scoring rubrics below before writing this paper. The evaluators will grade your paper based on the requirement in the rubrics. 


\section{Appendix J}

\section{The Cultural Analysis of Britain in Pride and Prejudice}

(Used as a Post-test for testing student's cultural understanding of literary texts)

\section{Introduction to the Assignment:}

This assignment asks you to write a critical essay that includes the cultural analysis of a literature text: Pride and Prejudice. This assignment is built on the premise that understanding a particular culture will help us better appreciate and comprehend that culture's literary productions, just as a careful reading of a literary text may lead us to better understand the culture from which it emerged.

For the purposes of this assignment, the definition of "cultural analysis" is large and open-ended. It means making connections between the texts we've read and the cultural contexts in which those texts emerged; it means making connections between texts and the cultural contexts in which those texts circulate. It does not exclude the formal or internal analysis of a text (indeed some of the very best cultural criticism uses formal analysis of literary texts); but cultural analysis moves beyond the boundaries of the text itself to establish links among texts, values, institutions, groups, practices, and people.

\section{Specific Directions:}

In Pride and Prejudice, Marriage is seemingly always on the minds of the characters, and this is telegraphed to us in the very famous line that opens the novel, "It is a truth universally acknowledge, that a single man in possession of a good fortune must be in want of a wife." These characters are either wishing of it, despairing of it ever happening, suffering from the lack of opportunity or, for those already married, reacting to their situation in life. Jane Austen provides a wide array of marriages to examine in this novel and the social satire, on first reading, often disguises the historical context of these marriages. Read through Pride and Prejudice, Keeping your attention focused on the theme of marriage and the elements of culture related to the marriage (for example, parental authority, social status, economic security, and pedigree, class consciousness). Then depict the cultural influences on the choice of marriage for the characters in Pride and Prejudice, analyze the possible causes of the characters' choice/values in his/her marriage. You can choose any character you are interested focusing the topic of marriage for the paper. In this study, you need to contextualize the British social culture for marriage in the Austen's time ( $18^{\text {th }}$ Century). You will need use a primary or secondary sources to help you secure you own grasp on the main points of values towards marriage in Britain.

The specific form and content of your cultural analysis paper should include the following aspects: (a) customs and beliefs towards marriage (b) gender roles concerning marriage; (c) economic status concerning marriage; (d) politics 
concerning marriage; (e) free will concerning marriage. I suggest you to have subtopics of these aspects while writing the paper.

Your papers may be deeply informed by a theory or may not. Some will want to develop a very precise idea of what "cultural analysis" means; others will not. Some will use a great deal of historical research; others only a little. Some papers will use mostly primary documents to construct an understanding of a British cultural context; others will rely on secondary sources; and others may use a mix of both. All these papers, however, must use documents and sources beyond the literary text itself.

What-I'm-Really-Looking-For. Just so you know, when I'm reading these papers, I'll be asking myself the following questions:

- Does the paper move beyond a formal analysis of a text in isolation?

- Does it focus on the literature and culture of Britain in the Austen's Time?

- Does it make links between texts and values, institutions, groups, practices, or people?

- Does the paper make specific and interesting claims about the text(s) and culture( $s)$ being examined?

- Does it explain in a clear and persuasive manner its interpretation of those texts and contexts?

- Does it support that interpretation with judiciously chosen evidence?

- Is it organized in a way that makes clear (rather than detracts from) the argument's major claims and emphases?

- Does it acknowledge its primary and secondary sources using a bibliography and a clear and consistent style of documentation?

\section{Requirements:}

Format: The paper should be at least five pages writing pieces. I value the quality of the paper, five-pages paper is only estimation of a quality paper for an English major. You are highly recommended to write a paper more than 5 page, if you have a lot of meaningful words to express. Use the Time New Roman 12 Font. Double Spaces. Delivery of Paper: You should submit a digital version to the instructor's email within one week [It is highly encouraged that a class volunteer could help collect all the digital paper in a package, and then send them(in WinRAR package) to the instructor via email at a designated time]. The instructor may also require you to bring a paper copy to the class (Specific submission directions will be discussed in the class).

Notes: Do not forget to write down your name as well the class at the beginning of the paper in the digital version.

Due Date: You will have two weeks to complete the paper after the initial announcement of assignment from the instructor.

The deadline for submitting a digital copy of this assignment is by 11:30pm Nov. 24,(Tues.) 2015.

The Assignment Evaluation: Please make sure you read the scoring rubrics below before writing this paper. The evaluators will grade your paper based on the requirement in the rubrics. 


\section{Appendix K}

\section{Scoring Rubrics for Cultural Analysis Essay}

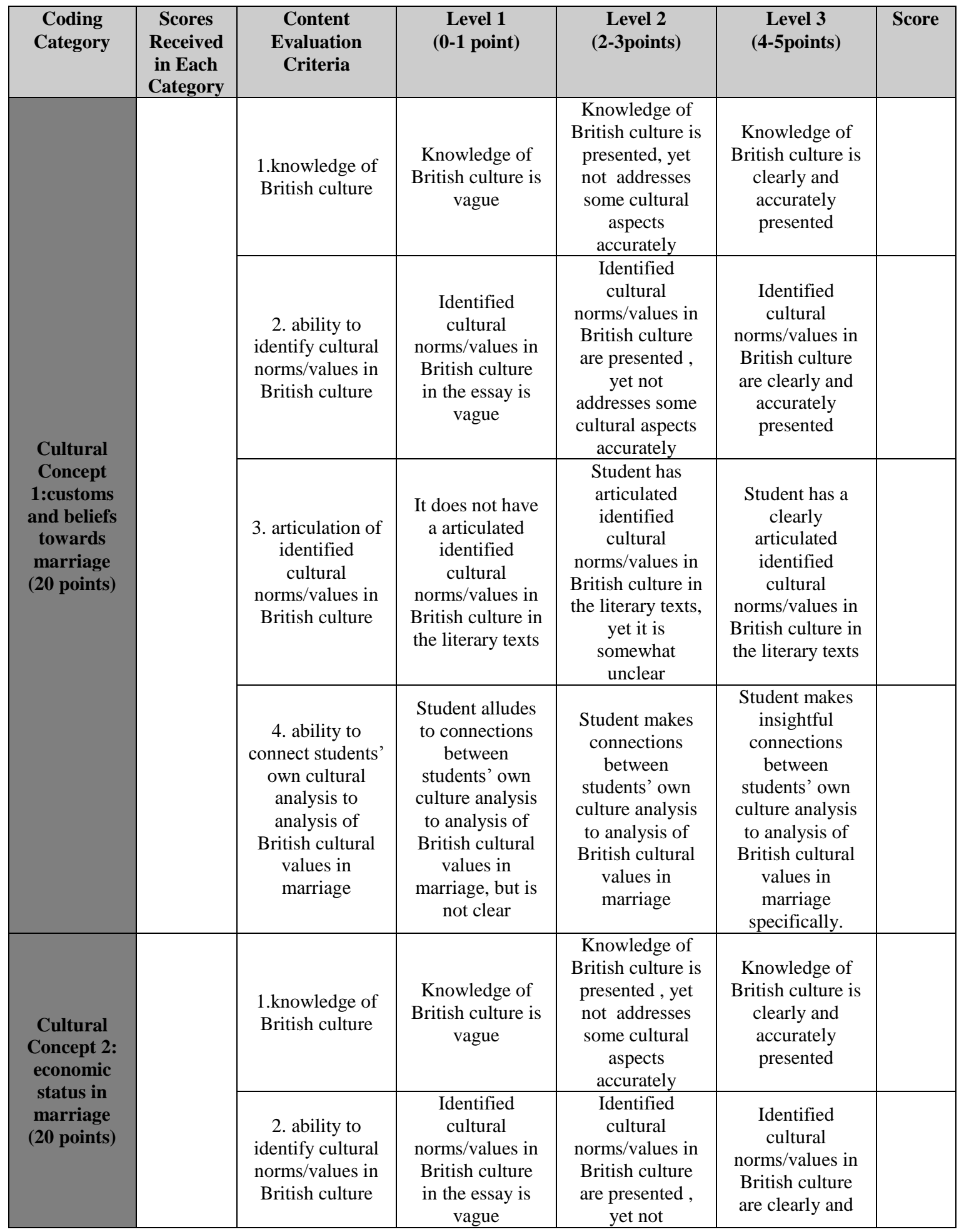




\begin{tabular}{|c|c|c|c|c|}
\hline & & & $\begin{array}{l}\text { addresses some } \\
\text { cultural aspects } \\
\text { accurately }\end{array}$ & $\begin{array}{l}\text { accurately } \\
\text { presented }\end{array}$ \\
\hline & $\begin{array}{l}\text { 3. articulation of } \\
\text { identified } \\
\text { cultural } \\
\text { norms/values in } \\
\text { British culture }\end{array}$ & $\begin{array}{l}\text { It does not have } \\
\text { a articulated } \\
\text { identified } \\
\text { cultural } \\
\text { norms/values in } \\
\text { British culture in } \\
\text { the literary texts }\end{array}$ & $\begin{array}{c}\text { Student has } \\
\text { articulated } \\
\text { identified } \\
\text { cultural } \\
\text { norms/values in } \\
\text { British culture in } \\
\text { the literary texts, } \\
\text { yet it is } \\
\text { somewhat } \\
\text { unclear }\end{array}$ & $\begin{array}{c}\text { Student has a } \\
\text { clearly } \\
\text { articulated } \\
\text { identified } \\
\text { cultural } \\
\text { norms/values in } \\
\text { British culture in } \\
\text { the literary texts }\end{array}$ \\
\hline & $\begin{array}{l}\text { 4. ability to } \\
\text { connect students' } \\
\text { own cultural } \\
\text { analysis of } \\
\text { British cultural } \\
\text { values in } \\
\text { marriage }\end{array}$ & $\begin{array}{l}\text { Student alludes } \\
\text { to connections } \\
\text { between } \\
\text { students' own } \\
\text { culture analysis } \\
\text { to analysis of } \\
\text { British cultural } \\
\text { values in } \\
\text { marriage, but is } \\
\text { not clear }\end{array}$ & $\begin{array}{l}\text { Student makes } \\
\text { connections } \\
\text { between } \\
\text { students' own } \\
\text { culture analysis } \\
\text { to analysis of } \\
\text { British cultural } \\
\text { values in } \\
\text { marriage }\end{array}$ & $\begin{array}{l}\text { Student makes } \\
\text { insightful } \\
\text { connections } \\
\text { between } \\
\text { students' own } \\
\text { culture analysis } \\
\text { to analysis of } \\
\text { British cultural } \\
\text { values in } \\
\text { marriage } \\
\text { specifically. }\end{array}$ \\
\hline & $\begin{array}{l}\text { 1.knowledge of } \\
\text { British culture }\end{array}$ & $\begin{array}{c}\text { Knowledge of } \\
\text { British culture is } \\
\text { vague }\end{array}$ & $\begin{array}{l}\text { Knowledge of } \\
\text { British culture is } \\
\text { presented, yet } \\
\text { not addresses } \\
\text { some cultural } \\
\text { aspects } \\
\text { accurately }\end{array}$ & $\begin{array}{l}\text { Knowledge of } \\
\text { British culture is } \\
\text { clearly and } \\
\text { accurately } \\
\text { presented }\end{array}$ \\
\hline Cultural & $\begin{array}{l}\text { 2. ability to } \\
\text { identify cultural } \\
\text { norms/values in } \\
\text { British culture }\end{array}$ & $\begin{array}{c}\text { Identified } \\
\text { cultural } \\
\text { norms/values in } \\
\text { British culture } \\
\text { in the essay is } \\
\text { vague }\end{array}$ & $\begin{array}{c}\text { Identified } \\
\text { cultural } \\
\text { norms/values in } \\
\text { British culture } \\
\text { are presented, } \\
\text { yet not } \\
\text { addresses some } \\
\text { cultural aspects } \\
\text { accurately } \\
\end{array}$ & $\begin{array}{c}\text { Identified } \\
\text { cultural } \\
\text { norms/values in } \\
\text { British culture } \\
\text { are clearly and } \\
\text { accurately } \\
\text { presented }\end{array}$ \\
\hline $\begin{array}{l}\text { Concept 3: } \\
\text { economic } \\
\text { status in } \\
\text { marriage } \\
(20 \text { points })\end{array}$ & $\begin{array}{l}\text { 3. articulation of } \\
\text { identified } \\
\text { cultural } \\
\text { norms/values in } \\
\text { British culture }\end{array}$ & $\begin{array}{l}\text { It does not have } \\
\text { a articulated } \\
\text { identified } \\
\text { cultural } \\
\text { norms/values in } \\
\text { British culture in } \\
\text { the literary texts }\end{array}$ & $\begin{array}{c}\text { Student has } \\
\text { articulated } \\
\text { identified } \\
\text { cultural } \\
\text { norms/values in } \\
\text { British culture in } \\
\text { the literary texts, } \\
\text { yet it is } \\
\text { somewhat } \\
\text { unclear }\end{array}$ & $\begin{array}{c}\text { Student has a } \\
\text { clearly } \\
\text { articulated } \\
\text { identified } \\
\text { cultural } \\
\text { norms/values in } \\
\text { British culture in } \\
\text { the literary texts }\end{array}$ \\
\hline & $\begin{array}{l}\text { 4. ability to } \\
\text { connect students' } \\
\text { own cultural } \\
\text { analysis of } \\
\text { British cultural } \\
\text { values in } \\
\text { marriage }\end{array}$ & $\begin{array}{l}\text { Student alludes } \\
\text { to connections } \\
\text { between } \\
\text { students' own } \\
\text { culture analysis } \\
\text { to analysis of } \\
\text { British cultural } \\
\text { values in } \\
\text { marriage, but is } \\
\text { not clear }\end{array}$ & $\begin{array}{l}\text { Student makes } \\
\text { connections } \\
\text { between } \\
\text { students' own } \\
\text { culture analysis } \\
\text { to analysis of } \\
\text { British cultural } \\
\text { values in } \\
\text { marriage }\end{array}$ & $\begin{array}{l}\text { Student makes } \\
\text { insightful } \\
\text { connections } \\
\text { between } \\
\text { students' own } \\
\text { culture analysis } \\
\text { to analysis of } \\
\text { British cultural } \\
\text { values in }\end{array}$ \\
\hline
\end{tabular}




\begin{tabular}{|c|c|c|c|c|}
\hline & & & & $\begin{array}{c}\text { marriage } \\
\text { specifically. }\end{array}$ \\
\hline \multirow{4}{*}{$\begin{array}{l}\text { Cultural } \\
\text { Concept } 4: \\
\text { politics in } \\
\text { marriage } \\
\text { (20 points) }\end{array}$} & $\begin{array}{l}\text { 1.knowledge of } \\
\text { British culture }\end{array}$ & $\begin{array}{c}\text { Knowledge of } \\
\text { British culture is } \\
\text { vague }\end{array}$ & $\begin{array}{l}\text { Knowledge of } \\
\text { British culture is } \\
\text { presented, yet } \\
\text { not addresses } \\
\text { some cultural } \\
\text { aspects } \\
\text { accurately }\end{array}$ & $\begin{array}{l}\text { Knowledge of } \\
\text { British culture is } \\
\text { clearly and } \\
\text { accurately } \\
\text { presented }\end{array}$ \\
\hline & $\begin{array}{l}\text { 2. ability to } \\
\text { identify cultural } \\
\text { norms/values in } \\
\text { British culture }\end{array}$ & $\begin{array}{c}\text { Identified } \\
\text { cultural } \\
\text { norms/values in } \\
\text { British culture } \\
\text { in the essay is } \\
\text { vague }\end{array}$ & $\begin{array}{c}\text { Identified } \\
\text { cultural } \\
\text { norms/values in } \\
\text { British culture } \\
\text { are presented, } \\
\text { yet not } \\
\text { addresses some } \\
\text { cultural aspects } \\
\text { accurately }\end{array}$ & $\begin{array}{c}\text { Identified } \\
\text { cultural } \\
\text { norms/values in } \\
\text { British culture } \\
\text { are clearly and } \\
\text { accurately } \\
\text { presented }\end{array}$ \\
\hline & $\begin{array}{l}\text { 3. articulation of } \\
\text { identified } \\
\text { cultural } \\
\text { norms/values in } \\
\text { British culture }\end{array}$ & $\begin{array}{l}\text { It does not have } \\
\text { a articulated } \\
\text { identified } \\
\text { cultural } \\
\text { norms/values in } \\
\text { British culture in } \\
\text { the literary texts }\end{array}$ & $\begin{array}{c}\text { Student has } \\
\text { articulated } \\
\text { identified } \\
\text { cultural } \\
\text { norms/values in } \\
\text { British culture in } \\
\text { the literary texts, } \\
\text { yet it is } \\
\text { somewhat } \\
\text { unclear } \\
\end{array}$ & $\begin{array}{c}\text { Student has a } \\
\text { clearly } \\
\text { articulated } \\
\text { identified } \\
\text { cultural } \\
\text { norms/values in } \\
\text { British culture in } \\
\text { the literary texts }\end{array}$ \\
\hline & $\begin{array}{l}\text { 4. ability to } \\
\text { connect students' } \\
\text { own cultural } \\
\text { analysis of } \\
\text { British cultural } \\
\text { values in } \\
\text { marriage }\end{array}$ & $\begin{array}{l}\text { Student alludes } \\
\text { to connections } \\
\text { between } \\
\text { students' own } \\
\text { culture analysis } \\
\text { to analysis of } \\
\text { British cultural } \\
\text { values in } \\
\text { marriage, but is } \\
\text { not clear }\end{array}$ & $\begin{array}{l}\text { Student makes } \\
\text { connections } \\
\text { between } \\
\text { students' own } \\
\text { culture analysis } \\
\text { to analysis of } \\
\text { British cultural } \\
\text { values in } \\
\text { marriage }\end{array}$ & $\begin{array}{l}\text { Student makes } \\
\text { insightful } \\
\text { connections } \\
\text { between } \\
\text { students' own } \\
\text { culture analysis } \\
\text { to analysis of } \\
\text { British cultural } \\
\text { values in } \\
\text { marriage } \\
\text { specifically. }\end{array}$ \\
\hline \multirow{3}{*}{$\begin{array}{l}\text { Cultural } \\
\text { Concept 5: } \\
\text { Free will in } \\
\text { marriage } \\
(20 \text { points })\end{array}$} & $\begin{array}{l}\text { 1.knowledge of } \\
\text { British culture }\end{array}$ & $\begin{array}{l}\text { Knowledge of } \\
\text { British culture is } \\
\text { vague }\end{array}$ & $\begin{array}{l}\text { Knowledge of } \\
\text { British culture is } \\
\text { presented, yet } \\
\text { not addresses } \\
\text { some cultural } \\
\text { aspects } \\
\text { accurately }\end{array}$ & $\begin{array}{l}\text { Knowledge of } \\
\text { British culture is } \\
\text { clearly and } \\
\text { accurately } \\
\text { presented }\end{array}$ \\
\hline & $\begin{array}{l}\text { 2. ability to } \\
\text { identify cultural } \\
\text { norms/values in } \\
\text { British culture }\end{array}$ & $\begin{array}{c}\text { Identified } \\
\text { cultural } \\
\text { norms/values in } \\
\text { British culture } \\
\text { in the essay is } \\
\text { vague }\end{array}$ & $\begin{array}{c}\text { Identified } \\
\text { cultural } \\
\text { norms/values in } \\
\text { British culture } \\
\text { are presented, } \\
\text { yet not } \\
\text { addresses some } \\
\text { cultural aspects } \\
\text { accurately }\end{array}$ & $\begin{array}{c}\text { Identified } \\
\text { cultural } \\
\text { norms/values in } \\
\text { British culture } \\
\text { are clearly and } \\
\text { accurately } \\
\text { presented }\end{array}$ \\
\hline & $\begin{array}{l}\text { 3. articulation of } \\
\text { identified } \\
\text { cultural } \\
\text { norms/values in } \\
\text { British culture }\end{array}$ & $\begin{array}{l}\text { It does not have } \\
\text { a articulated } \\
\text { identified } \\
\text { cultural } \\
\text { norms/values in }\end{array}$ & $\begin{array}{c}\text { Student has } \\
\text { articulated } \\
\text { identified } \\
\text { cultural } \\
\text { norms/values in }\end{array}$ & $\begin{array}{l}\text { Student has a } \\
\text { clearly } \\
\text { articulated } \\
\text { identified } \\
\text { cultural }\end{array}$ \\
\hline
\end{tabular}




\begin{tabular}{|c|c|c|c|c|}
\hline & & $\begin{array}{l}\text { British culture in } \\
\text { the literary texts }\end{array}$ & $\begin{array}{c}\text { British culture in } \\
\text { the literary texts, } \\
\text { yet it is } \\
\text { somewhat } \\
\text { unclear }\end{array}$ & $\begin{array}{l}\text { norms/values in } \\
\text { British culture in } \\
\text { the literary texts }\end{array}$ \\
\hline & $\begin{array}{l}\text { 4. ability to } \\
\text { connect students' } \\
\text { own cultural } \\
\text { analysis of } \\
\text { British cultural } \\
\text { values in } \\
\text { marriage }\end{array}$ & $\begin{array}{l}\text { Student alludes } \\
\text { to connections } \\
\text { between } \\
\text { students' own } \\
\text { culture analysis } \\
\text { to analysis of } \\
\text { British cultural } \\
\text { values in } \\
\text { marriage, but is } \\
\text { not clear }\end{array}$ & $\begin{array}{l}\text { Student makes } \\
\text { connections } \\
\text { between } \\
\text { students' own } \\
\text { culture analysis } \\
\text { to analysis of } \\
\text { British cultural } \\
\text { values in } \\
\text { marriage }\end{array}$ & $\begin{array}{l}\text { Student makes } \\
\text { insightful } \\
\text { connections } \\
\text { between } \\
\text { students' own } \\
\text { culture analysis } \\
\text { to analysis of } \\
\text { British cultural } \\
\text { values in } \\
\text { marriage } \\
\text { specifically. }\end{array}$ \\
\hline $\begin{array}{c}\text { Total Scores } \\
\text { for the } \\
\text { Essay } \\
\end{array}$ & & & & \\
\hline
\end{tabular}


Appendix L

\title{
CompTest1
}

\author{
安徽农业大学 2014-2015 学年第一学期 \\ 《英国文学 $I 》$ 试卷 $(A$ 卷)
}

考试形式:闭卷笔试 试卷总分： 100 分

适用专业 : 13 级英语

\begin{tabular}{|c|c|c|c|c|c|c|}
\hline 题号 & - & 二 & 三 & 四 & 五 & 总分 \\
\hline 得分 & & & & & & \\
\hline
\end{tabular}

\section{Part I}

Direction: Select one from the four choices of each item the one that best answers the question or completes the statement.

\begin{tabular}{|l|l|}
\hline 得分 & 评阅人 \\
\hline & \\
\hline
\end{tabular}

\section{I: Cultural and Historical Context (25 points, 2.5 points each)}

1.__ a typical example of old English poetry, is regarded today as the national epic of the Anglo-Saxons.
A.The Canterbury Tales
B.The Ballad of Robin Hood 


\section{C.The Song of Beowulf $\quad$ D.Sir Gawain and the Green Kinght}

2. The prevailing form of Medieval English literature is the
A. French
B. Latin C. Romance
D. Science

3. Which of the following historical events does not directly help to stimulate the rising of the Renaissance Movement?
A. The rediscovery of ancient Greek and Roman culture
B. The new discoveries in geography and astrology;
C. The Glorious Revolution
D. The religious reformation and the economic expansion

4.Generally, the Renaissance refers to the period between the $14^{\text {th }}$ and mid- $17^{\text {th }}$ centuries, its essence is
A.science
B.philosophy
C.arts
D.humanism

5. The English Renaissance period was an age of

A. Poetry and drama; B. Drama and Novel; C.NoveL and poetry; D. Romance and Poetry

6. About the Renaissance humanists which of the following statements is true?
A. They thought money and social status was the measure of all things.
B. They thought people were largely subordinated to the ruling class without any freedom and independence.
C. They couldn't see the human values in their works. 
D. They emphasized the dignity of human beings and the importance of the present

life

7. The following are the main qualities of Spenser's poetry except

A. Perfect melody; B. rare sense of beauty; C dedicated idealism; D bitter irony

8. Paradise Lost is actually a story taken from

A. The Renaissance; B. The Old Testament; C. Greek Mythology; D. The New

Testament

9. John Donne is the leading figure of

A. Lake Poets; B. Graveyard School; C. Satanic poets; D. Metaphysical School.

10 A poet perfected the blank verse and made it the principal medium of English

drama. The poet is

A. William Shakespeare; B. Christopher Marlowe; C. Geoffrey Chaucer; D. John Milton

II: Identification of Themes, Purposes, Plot Development (25 points, 2.5

points each)

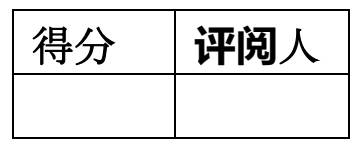

11. In his tragedy Romeo and Juliet, Shakespeare eulogizes

a. the faithfulness of love

b. the spirit of pursuing happiness

c. the heroine's great beauty, wit and loyalty 
d. both a and b

12."So long as men can breathe, or eyes can see,/So long lives this, and this gives life to thee."(Shakespeare, Sonnet18)What does“this"refer to ?
A.Lover.
B.Time.
C.Summer.
D.Poetry.

13. As to the great tragedy Hamlet, which of the following is not true?

A. The timeless appeal of this mighty drama lies in its combination of intrigue, emotional conflict and searching philosophic melancholy.

B. The bare outline of the play is based on a widespread legend in northern Europe.

C. The whole story of the play is created by Shakespeare himself.

D. In it, Shakespeare condemns the hypocrisy and treachery and general corruption at the royal court.

14. About Shakespeare's romantic comedies, which of the following is true? A. He takes an optimistic attitude toward love and truth.

B. The romantic elements are not brought into full play at all.

C. He presents the patriotic spirit when engaging intellectual excitement and emotion.

D. There is a wonderful balance of characters. 
15. In Hamlet's "To be or not to be" soliquy (ACT III,Scene 1), one of the courses of action he considers is "taking arms against a sea of troubles", meaning he would
A. stage a military coup against Claudius
B. finance an invading army's attack $\mathrm{h}$ upon Claudius
C. take immediate and decisive action against Cludius
D. take Claudius' arm in a symbolic amputatio

16. Paradise Lost tells the story of
A. a young prince's revenge on his father's murderer
B. the expulsion of Adam and Eve out of the garden of Eden
C. Satan's rebellion against God
D. both B and C

17. “O prince, $\mathrm{O}$ chief of my throned powers, / That led the embattled seraphim to war/Under thy conduct, and in dreadful deeds/Fearless, endangered Heaven's perpetual king"In the third line of the above passage quoted from Milton's Paradise Lost, the phrase"thy conduct"refers to conduct.
A.God's
B.Satan's
C.Adam's
D.Eve's

18. Which of the following characters bears the most resemblance with its creator in John Milton's works?
A. Satan in Paradise Lost
B. God in Paradise Lost
C. Samson in Samson Agonistes
D. Christ in Paradise Regained 
19. The predominated metaphor in The Pilgrim's Progress is that
A. Life is a journey
B. Life is a dream
C. Life is to endure hardship
D. none of the above

20. The Pilgrim's progress by John Bunyan is often said to be concerned with the search for
A.self-fulfillment
B.spiritual salvation
C.material wealth
D.universal truth

\section{III: Literary Analysis, Appreciation and Comprehension (50 points)}

\begin{tabular}{|l|l|}
\hline 得分 & 评阅人 \\
\hline & \\
\hline
\end{tabular}

A) Read the following selected literary pieces, and then answer the following questions. (The total points are 20 points. Five points for each question)

What a piece of work is man! How noble in reason! How infinite in faculties! In form and moving, how express and admirable! In action how like an angel! In apprehension, how like a god! The beauty of the world! The paragon of animals! And yet, to me, what is this quintessence of dust? Man delights not me; no, nor woman neither, though by your smiling you seem to say so 
1. Which of the following emotions are expressed most clearly in Hamelet's words?
A. Fury
B. Grief
C. Meancholy
D. Fear
E. Disgust

2. At first, Hamlet seems to think mankind is:
A. A DOOMED TO FAILURE
B. Capable of greatness bordering on perfection
C. Incapable of greatness bordering on perfection
D. Succeeding marvelously in God's plan

3. He concludes by declaring his:
A. Hatred of women
B. Satisfaction in being proven right about man's dismal fate
C. Belief that mean are inherently better than women
D. Distaste at the current example being set by those around him

4. This passage could be described as the summary of
A. Hamlet's Renaissance Views
B. Hamlet's antiquated (陈旧古老的) opinions
C. Claudia's confidence in Hamlet's eventual failure
D. Ophela's distress, first with Hamlet's madness, and then with Gertrude's role

B) Analyze the characteristics of Hamlet based on your understandings of the whole play Hamlet. How does the characteristics of Hamlet reflect the humanism in Shakespeare's time ?(10 points)

C) Read the following selected literary pieces, and then answer the following questions. (The total points are 20 points. Five points for each question ) 


\section{Sonnet 18}

Shall I compare thee to a summer's day?

Thou art more lovely and more temperate:

Rough winds do shake the darling buds of May,

And summer's lease hath all too short a date,

Sometimes too hot the eye of heaven shines, Line 5

And often is his gold complexion dimmed,

And every fair from fair sometime declines,

By chance, or nature's changing course, untrimmed.

But thy eternal summer shall not fade,

Nor lose possession of that fair thou ow'st ........ Line 10

When in eternal lines to time thou grow'st.

So long as men can breathe or eyes can see,

So long lives this, and this gives life to thee

1.The poem is concerned primarily with the
A. Eternal nature of love
B. Idea that nature never changes
C. Transient nature of flowers
D. Idea that humanity created eternal objects
E. Eternal nature of summer

\section{EXCEPT}

2.The metaphoric use of "a summer's day" (line 1-2) suggests all of the following 

A. How much like a summer's day is the person addressed
B. The shortness of life
C. The contrast between the fleeting days of summer and the eternal quality of love
D. The ugliness of life
E. Continuous, but ever-changing nature

3.Line 13 and 14 of the poem can best be described as
A. Blank verse
B. Rhymed triplet
C. Free verse
D. Rhymed couplet

4.Lines 5-6 employ the figure of speech called
A. Personification
B. Allusion
C. Alliteration
D. Allegory 


\section{Answer Key for CompTest1:}

\section{$1-5$ CCCDA}

6-10DBBDB

11-15DDCAC

16-20DBCCB

HAMLET 1-4 CBDA

SONNET 1-4 ADDA

Literary Analysis on Hamlet's Characteristics Key Points:

(a)Hamlet is an elusive and mysterious character that is philosophical, contemplative, obsessive, impulsive, melancholy, intelligent and careless. (3 points).

Students should at least summarize three main characteristics of Hamlet based on analyzing the literary texts.

(b) In the context of the English Renaissance, a humanist may have a belief in self, human worth, and individual dignity. ( 2 points, students should point out the characteristics of humanist belief)

Here are some examples of how these characteristics are illustrated in Hamlet:

Humanist philosophy: In Act II, Scene 2, Line 311, Hamlet asks: "What a piece of work is a man, how noble in reason, how infinite in faculties..." In this speech, you can see a clear assertion of humanist ideas about the uniqueness and extraordinary abilities of the human mind. ( 3 points, students will receive the full points if they can relate the characteristics of humanism in the Hamlet texts. 


\section{Appendix M}

\section{CompTest2}

安徽农业大学 2014-2015 学年第一学期

\section{《英国文学 II 》试卷（A 卷）}

考试形式:闭卷笔试 试卷总分： 100 分

适用专业： 13 级英语

\begin{tabular}{|l|l|l|l|l|l|l|}
\hline 题 号 & - & 二 & 三 & 四 & 五 & 总分 \\
\hline 得 分 & & & & & & \\
\hline
\end{tabular}

\begin{tabular}{|l|l|}
\hline 得 & 评阅人 \\
分 & \\
\hline & \\
\hline
\end{tabular}

\section{I: Cultural and Historical Context (25 points, 2.5 points each)}

1. The $18^{\text {th }}$-century England is known as ( )

A. The Age of Puritanism

B. The Age of Reason

C. The Era of Capitalism

D. The Age of Glory

2. The enlighteners placed much emphasis on reason, because they thought ( )

A. Superstition was above reason and rationality

B. Reason and emotion both could lead to truth and justice 
C. Reason or rationality should the only, the final cause of any human thought and activities.

D. Equality and science is contrary to and rationality.

3. Which of the following statements is true according to the principles of the neoclassicists?

A. All forms of literature were to be modeled after the classical works of the ancient Greek and Roman writers

B. They tried to delight, instruct and correct human beings as social animals

C. They tried to develop a polite, urbane, witty and intellectual art

D. All of the above

4. The British bourgeois or middle class believed in the following notions EXCEPT
A. Self-esteem
B. Self-reliance
C. Self-restraint
D. Hard work

5. In the field of literature, the Enlightenment Movement brought about the tendency of ( )
A. realism
B. Puritanism
C. Neoclassicism
D. Romanticism

6. In the $18^{\text {th }}$ century, ___ found its expression chiefly in poetry, especially that of William Blake and Robert Burns

A. Neo-classicism; B. Realism; C. Sentimentalism; D. Pre-romanticism

7. Romanticism was a literary trend prevailing in English during the period from 1798 to 1832 . Romantic writer ( )

A. Paid great attention to the spiritual and emotional life of man

B. Were discontent with the development of industrialism and capitalism, and presented the social evils minutely in their works

C. Took pains to portray a world of harmony and balance

D. Tended to glorify Rome and advocated rational Italian and French art as superior the native traditions

8. The 18th century witnessed a new literary form-the modern English novel, which, contrary to the medieval romance, gives a presentation of life of the common people.

A. Romantic; B. Realistic; C. Prophetic, D. Idealistic

9. The literary form which is fully developed and the most flourishing during the Romantic period is 


\section{A. Prose; B. Drama; C. Novel; D. Poetry}

10. Austen's main literary concern is about

A. human beings in their personal relationships

B. human society of the 18th century

C. the follies and illusions of mankind

D. order, reason proportion and gracefulness

\section{II: Identification of Themes, Purposes, Plot Development (25 points, 2.5}

\section{points each)}

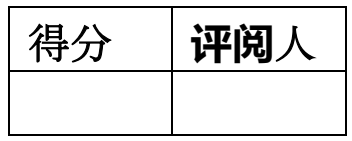

who

11.The hero in Robinson Crusoe is a typical $18^{\text {th }}$-century English middle-class man

A. has a great capacity for work, inexhaustible energy, courage, patience and persistence in overcoming obstacles and struggling against the hostile natural environment

B. has strong will but can't endure life's loneliness

C. has a great capacity for work, but is frightened by the hostile natural environment

D. thinks all the people are born equal

12. Lilliput is in Gulliver's Travels by Jonathan Swift.

A. the name of the hero who made deep-sea voyages

B. an imaginary island inhabited by people not more than six inches high

C. a minor character who accompanied the hero during his voyages

D. the country of horses endowed with human intelligence

13. According to subjects, Wordsworth's short poems can be classified into two groups:

A. poems about nature and poems about politics

B. poems about nature and poems about human life

C. poems about love and poems about beauty

D. poems about society and poems about history.

14. Which of the following descriptions of Gothic Novels is not correct? 

A. It predominated in the early eighteenth century
B. It was one phase of the Romantic movement
C. Its principal elements are violence, horror and the supernatural
D Works like the Mysteries of Udolpho and Frankenstein are typical Gothic

romantic novel

15 "Tiger! Tiger! Buring bright/ In the forests of the night, what immortal hand or eye/Could frame thy fearful symmetry?" (“The Tiger” by William Blake) The above lines $(\quad)$

A. Describe the tiger's fierce eyes and forceful hands at night

B. Express the poet's curiosity for the skillful creation of the tiger

C. Express the poet's surprise at the sight of the tiger's well-proportioned body

D. Express the poet's terror at the sight of the tiger in the forest at night

16. The assertion that poetry originates from "emotion recollected in tranquility" belongs to
A. William Wordsworth
B. Samuel Taylor Coleridge
C. Robert Southey
D. William Blake

1. "Drive my dead thoughts over the universe Like withered leaves to quicken a new birth." (Percy Bysshe Shelly, "Ode to the West Wind")

What rhetorical device does the poet use in the quoted linnes?
A. Synecdoche
B. Metaphor
C. Simile
D. Onomatopoeia 
2. Through the character of Elizabeth, Jane Austin emphasizes the importance of
A. Marriage
B. Physical attractiveness
C. Independence and self-confidence
D. Submissive character

3. The subject matter in Jane Austen's novels is very limited. It is confined to the description of
A. The life of English rural gentry's class
B. English urban people
C. London society
D. English farmers
8.

4. "She is all goodness, never speaks badly of anyone, is patient and gentle." Which of the Bennet daughters is being described?
A. Jane
B. Elizabeth
C. Kitty
D. Lydia

\section{III: Literary Analysis, Appreciation and Comprehension (50 points)}

\begin{tabular}{|l|l|}
\hline 得分 & 评阅人 \\
\hline & \\
\hline
\end{tabular}

A) Directions: Read the following passage carefully before you choose your answers. from: Volume I, chapter 18. The total points are 20 points. Five points for each question.

She then changed the discourse to one more gratifying to each, and on which there could be no difference of sentiment. Elizabeth listened with delight to the happy, though 
modest hopes which Jane entertained of Bingley's regard, and said all in her power to heighten her confidence in it. On their being joined by Mr. Bingley himself, Elizabeth withdrew to Miss Lucas; to whose inquiry after the pleasantness of her last partner she had scarcely replied, before Mr. Collins came up to them and told her with great exultation that he had just been so fortunate as to make a most important discovery. "I have found out," said he, "by a singular accident, that there is now in the room a near relation of my patroness. I happened to overhear the gentleman himself mentioning to the young lady who does the honours of this house the names of his cousin Miss de Bourgh, and of her mother Lady Catherine. How wonderfully these sort of things occur! Who would have thought of my meeting with — perhaps — a nephew of Lady Catherine de Bourgh in this assembly!- I am most thankful that the discovery is made in time for me to pay my respects to him, which I am now going to do, and trust he will excuse my not having done it before. My total ignorance of the connection must plead my apology."

"You are not going to introduce yourself to Mr. Darcy?"

"Indeed I am. I shall intreat his pardon for not having done it earlier. I believe him to be Lady Catherine's nephew . It will be in my power to assure him that her ladyship was quite well yesterday se'nnight."

Elizabeth tried hard to dissuade him from such a scheme; assuring him that Mr. Darcy would consider his addressing him without introduction as an impertinent freedom, rather than a compliment to his aunt; that it was not in the least necessary there should be any notice on either side, and that if it were, it must belong to Mr. Darcy, the superior in consequence, to begin the acquaintance.-Mr. Collins listened to her with the determined air of following his own inclination and when she ceased speaking, replied thus, 
"My dear Miss Elizabeth, I have the highest opinion in the world of your excellent judgment in all matters within the scope of your understanding, but permit me to say that there must be a wide difference between the established forms of ceremony amongst the laity, and those which regulate the clergy; forgive me leave to observe that I consider the clerical office as equal in point of dignity with the highest rank in the kingdom - provided that a proper humility of behavior is at the same time maintained. You must therefore allow me to follow the dictates of my conscience on this occasion, which leads me to perform what I look on as a point of duty. Pardon me for neglecting to profit by your advice, which on every other subject shall be my constant guide, though in the case before us I consider myself more fitted by education and habitual study to decide on what is right than a young lady like yourself." And with a low bow he left her to attack Mr. Darcy, whose reception of his advances she eagerly watched, and whose astonishment at being so addressed was very evident.

21. What does Mr. Collins' plan to introduce himself to Mr. Darcy reveal about his character?
A. He is meticulous in his observation of social propriety.
B. He has an inflated opinion of his own social standing.
C. He is a fawning sycophant.
D. He is sensitive to the opinions and feelings of Elizabeth.
E. He has a keen sense of character.

22. When Mr. Collins tells Elizabeth that he has "the highest opinion in the world of [her] excellent judgment in all matters within the scope of [her] understanding" 
(lines 28 - 29), he implies that Elizabeth
A. is socially inferior.
B. is intellectually inferior.
C. is more educated than most women.
D. has foolishly determined to marry too far above her class.
E. has offended Mr. Collins's sense of social position.

23. How does Mr. Collins react to Elizabeth's rejection of his marriage proposal?

A. He is insulted and threatens to throw the family out of the house if she does not accept.

B. He thinks she is doing it to be flirtatious. It is beyond his comprehension to think he would be rejected.

C. He desperately wants to get married, so he offers to keep all of the other sisters in the house until they get married. He also offers to let Mrs. Bennet live there as long as she wishes.

D. He cheerfully reminds her that she has four sisters, and one of them will be smart enough to marry him. He also insinuates that she will not be welcome in the house after he is the owner.

24. The dashes and exclamation points used to describe Mr. Collins excitement at the proposition of meeting Mr. Darcy exemplify:
A. diction
B. irony 

C. detail
D. purpose
E. syntax

B) Elizabeth Bennet, the heroine in Pride and Prejudice, is often regarded as the most successful character created by Jane Austen. Analyzed Elizabeth's character based on the novel. (10 points)

Directions: Actively read each poem and then answer the questions that follow. (5 points Each)

\section{"I Wandered Lonely as a Cloud" - William Wordsworth}

I wandered lonely as a cloud

That floats on high o'er the vales and hills.

When all at once I saw a crowd,

A host, of golden daffodils.

Beside the lake, beneath the trees,

Fluttering and dancing in the breeze.

Continuous as the stars that shine

And twinkle on the milky way,

They stretched in never-ending line

Along the margin of a bay;

Ten thousand saw I at a glance, Tossing their heads in sprightly dance.

The waves beside them dances, but they

Outdid the sparking waves in glee:

A poet could not but be gay, 


$$
\begin{aligned}
& \text { In such a jocund company: } \\
& \text { I gazed - and gazed - but little thought } \\
& \text { What wealth the show to me had brought; }
\end{aligned}
$$

For oft, when on my couch I lie In vacant or in pensive mood,

They flash upon that inward eye

Which is the bliss of solitude:

And then my heart with pleasure fills,

And dances with the daffodils.

25. The tone of "I Wandered Lonely as a Cloud" can be best described as
a. joyful.
c.
angry.
b. sad.
d. comic.

26. The rhyme scheme of the first stanza of this poem is
a. abcabc.
c. ababcc.
b. abbacc.
d. abbacc.

27.Which word does Wordsworth use most often when describing action in the poem?
a.
running.
c. dancing.
b. sleeping.
d. falling.

28. Whose perspective does the poem represent?
a. the speaker's
c. the stars'
b. the daffodils'
d. the waves' 


\section{Answers Key for CompTest2:}

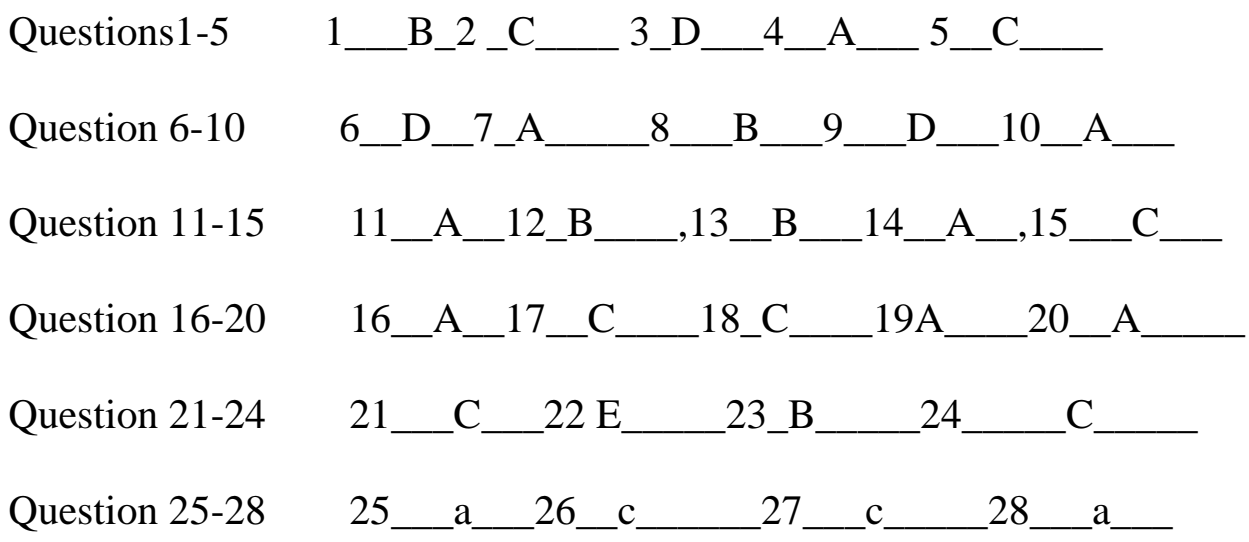

Answer Key for the literary analysis of Elizabeth's character: Elizabeth

Bennet is the most intelligent and quick-witted, and one of the most well-known female characters in English literature. Her admirable qualities are numerous - she is lovely, clever, and, in a novel defined by dialogue, she converses as brilliantly as anyone. Her honesty, virtue, and lively wit enable her to rise above the nonsense and bad behavior that pervade her class-bound and often spiteful society. Nevertheless, her sharp tongue and tendency to make hasty judgments often lead her astray; Pride and Prejudice essentially the story of how she (and her true love, Darcy) overcome all obstacles-including their own personal failings - to find romantic happiness. Elizabeth must not only cope with a hopeless mother, a distant father, two badly behaved younger siblings, and several snobbish, antagonizing females, she must also overcome her own mistaken impressions of Darcy, which initially lead her to reject his proposals of marriage. Her charms are sufficient to keep him interested, fortunately, while she navigates familial and social turmoil. As she gradually comes to recognize the nobility of Darcy's character, she realizes the error of her initial prejudice against him. 


\section{Appendix N}

\section{CompTest3}

\section{安徽农业大学 2015-2016 学年第一学期 \\ 《英国文学史及选读》试卷（A 卷）}

考试形式:闭卷笔试, 2 小时

适用专业：英语专业

试卷总分 : 100 分

考试日期 : 2015 年 12 月

\begin{tabular}{|l|l|l|l|l|l|l|}
\hline 题 号 & - & 二 & 三 & 四 & 五 总分 \\
\hline 得 分 & & & & & & \\
\hline
\end{tabular}

\begin{tabular}{|l|l|}
\hline 得分 & 评阅人 \\
\hline & \\
\hline
\end{tabular}

\section{I:Cultural and Historical Context.}

Directions: Each of the statements below is followed by four answers. Choose the one that would best complete the statement and write your choices on the answer sheet. (15points, 1.5 for each)

1. The piece of work that is commonly considered to be the beginning of the English literature is
A. Beowulf
B. The Canterbury Tales
C. Le Morte d'Arthur
D. Paradise Lost 
2. The essence of humanism is to

A. restore a medieval reverence for the church

B. avoid the circumstances of earthly life

C. explore the next world in which men could live after death

D. emphasize human qualities

3. One of the distinct features of the Elizabethan time is

A. the flourishing of the drama

B. the popularity of the realistic novel

C. the domination of the classical poetry

D. the close-down of all the theatres

4. The 18th century England is known as the in the history.
A. Renaissance
B. Classicism
C. Enlightenment
D. Romanticism

5. As to education, the enlighteners thought that

A. human beings were limited, dualistic, imperfect, and not capable of rationality and perfection through education

B. universal education was unnecessary

C. if the common people were well educated, there would be great chance for a democratic and equal human society

D. most of the human beings were perfect themselves, so only a few needed further education

6. In the following descriptions of the Neoclassical Period, which is wrong?

A. The Neoclassical Period is prior to the Romantic Period.

B. One major belief that the Neoclassicists held is the artistic ideals should be order, logic, restrained emotion \& accuracy

C. The modern English novel came into being in the Neoclassical Period.

D. Neoclassical Period is also known as the Age of Enlightenment.

7. The Romantic Movement expressed a more or less attitude toward the existing social and political conditions.
A. positive
B. negative
C. neutral
D. indifferent

8. For the Romantics, is not only the major source of poetic imagery, but also provides the dominant subject matter.
A. love
B. man
C. nature
D. death

9. In the Romantic period,
A. prose
B. poetry
C. fiction
D. play is the most prosperous literary form. 
10. As a leading Romanticist, Byron's chief contribution is his creation of the "Byronic hero", a
A. proud, strong-minded rebel under pressure
B. proud, mysterious rebel of noble birth
C. proud, selfish person with evil heart
D. proud, vindictive person without mercy

\begin{tabular}{|l|l|}
\hline 得分 & 评阅人 \\
\hline & \\
& \\
\hline
\end{tabular}

\section{II: Identification of Themes, Purpose, and Plot Development}

Directions: Each of the statements below is followed by four answers. Choose the one that would best complete the statement and write your choices on the answer sheet. (15points, 1.5for each)

11. What, according to Beowulf, is better than mourning a death?
A. Celebrating a birth
B. Avenging a death
C. Drinking one's sorrows away
D. Making peace with one's enemies

12. In Hamlet, Ophelia is most upset at Hamlet's apparent madness because she
A. drove him to it
B. loved him
C. recognizes her father's cruel intent
D. sees the folly of her ways

13. The theme of Hamlet's "To be or not to be" soliloquy is best described as A. A serious meditation on suicide

B. A lamentation over the loss of his one true love 
C.A general exposition on the best course of action when faced withed with a dilemma

D.A general exposition on the best method of riddling himself of Ophelia

14. The work that presented, for the first time in English literature, a comprehensive realistic picture of the medieval English society and created a whole gallery of vivid characters from all walks of life is most likely
A. William Langland's Pier the Plowman
B. Geoffery Chaucer's The Canterbury Tales
C. John Gower's Confession Amantis
D. Sir Gawain and the Green Knight

15. Which of the following statements best illustrates the theme of Shakespeare's Sonnet 18 ?
A. The speaker eulogizes the power of Nature.
B. The speaker satirizes human vanity.
C. The speaker praises the power of artistic creation.
D. The speaker meditates on man's salvation.

16. The social significance of Gulliver's Travels lies in

A. the devastating criticisms and satires of all aspects in the then English and European life

B. his artistic skill in making the story an organic whole

C. his central concern of study of human nature and life

D. both $\mathrm{b}$ and $\mathrm{c}$

17. The Houyhnhnms depicted in Gulliver's Travels are 

A. horses that are endowed with reason
B. pigmies that are endowed with admirable qualities
C. giants that are superior in wisdom
D. hairy, wild, low and despicable creatures who resemble human beings not only in appearance but also in some other ways

18. The hero in Robinson Crusoe is the prototype of
A. the empire builder
B. the pioneer colonist
C. the working people
D. both $a$ and $b$

19. In Pride and Prejudice, although Mrs. Bennet is an unwise and foolish woman, why can we understand her pursuit of rich young men for her daughters?

A. She wants to make sure they get better husbands than she did. She thinks she can ease some of the pain of her own mistake by helping them.

B. She made a promise to her dying mother that she would take care of her girls, and she is determined to fulfill her promise.

C. Mr. Bennet's estate is entailed on a distant male relative; therefore, his daughters will lose their home upon his death. She wants them to have secure futures.

D. A mother's success was based on the wealth of her daughters and the number of grandchildren she had. Since Mrs. Bennet is very conscious of social position, she wants to make sure that she is doing everything she can to assure herself of high social standing.

20. In Pride and Prejudice, Elizabeth remarks to Jane that "The more I see of the world, the more I am dissatisfied with it; and every day confirms my belief of the 
inconsistency of all human characters, and of the little dependence that can be placed on the appearance of either merit or sense."

This quotation is the best example of which of the following themes in the novel:

A. First impressions are often misleading.

B. Society's expectations have the ability to corrupt the idea of marriage.

C. The actions of one individual have an impact on that individual's society.

D. One's decisions should be based on morality and virtue, rather than social decorum.

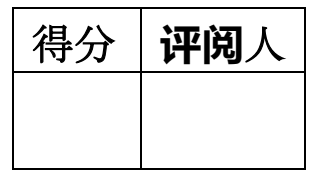

\section{Reading Comprehension}

Directions: This section includes three reading pieces. Read the selected literary scripts carefully and choose the one that would best answer to the question. Then write your choices on the answer sheet (30 points, 2 points for each)

a) Poetry Comprehension

Directions: Read the Ode to the West Wind, Stanza IV \& V, and then choose the best answer for Questions 21-24:

\section{Stanza IV}

If I were a dead leaf thou mightest bear;

If I were a swift cloud to fly with thee;

A wave to pant beneath thy power, and share

The impulse of thy strength, only less free

Than thou, O uncontrollable! If even 
I were as in my boyhood, and could be The comrade of thy wanderings over Heaven, As then, when to outstrip thy skiey speed Scarce seem'd a vision; I would ne'er have striven As thus with thee in prayer in my sore need.

Oh, lift me as a wave, a leaf, a cloud!

I fall upon the thorns of life! I bleed!

A heavy weight of hours has chain'd and bow'd One too like thee: tameless, and swift, and proud.

\section{Stanza V}

Make me thy lyre, even as the forest is:

What if my leaves are falling like its own!

The tumult of thy mighty harmonies

Will take from both a deep, autumnal tone,

Sweet though in sadness. Be thou, Spirit fierce,

My spirit! Be thou me, impetuous one!

Drive my dead thoughts over the universe

Like wither'd leaves to quicken a new birth!

And, by the incantation of this verse,

Scatter, as from an unextinguish'd hearth

Ashes and sparks, my words among mankind!

Be through my lips to unawaken'd earth

The trumpet of a prophecy! Oh Wind, 
If Winter comes, can Spring be far behind?

21. The poet says that as a young man he too was like the West wind, because he also was
A. Wide, swift and proud
B. Tame, swift and happy
C. Soft, red and alert
D. Quiet, quick and dangerous

22. Why is the West wind a trumpet of prophecy?
A. It brings rain
B. It blows over all regions
C. It brings the promise of spring
D. It brings warmth

23. What are 'the thorns of life?
A. The difficulties
B. The injuries
C. The evil people
D. The world

24. $\mathrm{O}$ wind, if winter comes, can spring be far behind? The mood of the poet is
A. Pessimism
B. Optimism
C. Realism
D. Determination

\section{b) Literary Criticism Comprehension}

Directions: Read Hamlet and His Problems and then choose the best answer for Questions 25-29.

We know that there was an older play by Thomas Kyd, that extraordinary dramatic (if not poetic) genius who was in all probability the author of two plays so dissimilar as 
The Spanish Tragedy and The Arden of Febersham; and what this play was like we can guess from three clues: from The Spanish Tragedy itself, from the tale of Belleforest upon which Kyd's Hamlet must have been based, and from a version acted in Germany in Shakespeare's lifetime which bears strong evidence of having been adapted from the earlier, not the later, play. From these three sources it is clear that in the earlier play the motive was simply a revenge motive; that the action or delay is caused, as in The Spanish Tragedy, solely by the difficulty of assassinating a monarch surrounded by guards; and that the "madness" of Hamlet was feigned in order to escape suspicion, and successfully. In the final play of Shakespeare, on the other hand, there is a motive which is more important than that of revenge, and which explicitly "blunts" the latter; the delay is unexplained on grounds of necessity or expediency; and the effect of "madness" is not to lull but arouse the king's suspicion. The alteration is not complete enough, however, to be convincing. Furthermore, there are verbal parallels so close to The Spanish Tragedy as to leave no doubt that in places Shakespeare was merely revising the text of Kyd. And finally there are unexplained scenes-the Polonius-Reynaldo scenes-for which there is little excuse; these scenes are not in the verse style of Kyd and not beyond doubt in the style of Shakespeare. These Mr. Robertson believes to be scenes in the original play of Kyd reworked by a third hand, perhaps Chapman, before Shakespeare touched the play.

From "Hamlet and His Problems," by T.S. Eliot.

25. The author suggests that Kyd's older play was called
A. The Spanish Tragedy
B. Belleforest
C. The Arden of Feversham
D. A German Tragedy
E. Hamlet 
26. The author suggest that Shakespeare's pay is a revision of
A. A German version of Kyd's.
B. The Arden of Feversham
C. The Spanish Tragedy
D. The Tale of Belleforest
E. His own earlier play

27. We can guess what the Kyd play was like through clues in

I. The Arden of Feversham

II. A German version in Shakespeare's time.

III. The Spanish Tragedy and the tale of Belleforest.
A. I and III only
B. I only
C. II only
D. III only
E. II and III only

28. Kyd's earlier play was a(n)

F. Historical saga

G. Revenge play

H. Chronicle

I. Morality play

29. Which of the following words best describe the author's tone?
A. Calm and objective
B. Pedagogical and dull
C. Condescending and lecturing
D. Learned and high-brow

\section{C) Literary Work Comprehension}

Directions: Read the following passage from chapter 32 in Pride and Prejudice carefully and then choose the best answer for Questions 30-35

Elizabeth was sitting by herself the next morning, and writing to Jane, while Mrs. Collins and Maria were gone on business into the village, when she was startled by a ring at the door, the certain signal of a visitor. As she had heard no carriage, she thought it not unlikely to be Lady Catherine, and under that apprehension was putting away her halffinished letter that she might escape all impertinent questions, when the door opened, and to her very great surprise, Mr. Darcy, and Mr. Darcy only, entered the room. 
He seemed astonished too on finding her alone, and apologized for his intrusion by letting her know that he had understood all the ladies to be within. They then sat down, and when her enquiries after Rosings were made, seemed in danger of sinking into total silence. It was absolutely necessary, therefore, to think of something, and in this emergency recollecting when she had seen him last in Hertfordshire, and feeling curious to know what he would say on the subject of their hasty departure, she observed,

"How very suddenly you all quitted Netherfield last November, Mr. Darcy! It must have been a most agreeable surprise to Mr. Bingley to see you all after him so soon; for, if I recollect right, he went but the day before. He and his sisters were well, I hope, when you left London."

"Perfectly so-I thank you."

She found that she was to receive no other answer-and, after a short pause, added, "I think I have understood that Mr. Bingley has not much idea of ever returning to Netherfield again?"

"I have never heard him say so; but it is probable that he may spend very little of his time there in future. He has many friends, and he is at a time of life when friends and engagements are continually increasing."

"If he means to be but little at Netherfield, it would be better for the neighbourhood that he should give up the place entirely, for then we might possibly get a settled family there. But perhaps Mr. Bingley did not take the house so much for the convenience of the neighbourhood as for his own, and we must expect him to keep or quit it on the same principle." 
"I should not be surprised," said Darcy, "if he were to give it up, as soon as any eligible purchase offers."

Elizabeth made no answer. She was afraid of talking longer of his friend; and, having nothing else to say, was now determined to leave the trouble of finding a subject to him.

He took the hint, and soon began with, "This seems a very comfortable house. Lady Catherine, I believe, did a great deal to it when Mr. Collins first came to Hunsford."

"I believe she did—and I am sure she could not have bestowed her kindness on a more grateful object."

"Mr. Collins appears very fortunate in his choice of a wife."

"Yes, indeed; his friends may well rejoice in his having met with one of the very few sensible women who would have accepted him, or have made him happy if they had.

My friend has an excellent understanding - though I am not certain that I consider her marrying Mr. Collins as the wisest thing she ever did. She seems perfectly happy, however, and in a prudential light, it is certainly a very good match for her."

"It must be very agreeable to her to be settled within so easy a distance of her own family and friends."

"An easy distance do you call it? It is nearly fifty miles."

"And what is fifty miles of good road? Little more than half a day's journey. Yes, I call it a very easy distance.” 
"I should never have considered the distance as one of the advantages of the match," cried Elizabeth. "I should never have said Mrs. Collins was settled near her family."

"It is a proof of your own attachment to Hertfordshire. Anything beyond the very neighbourhood of Longbourn, I suppose, would appear far."

As he spoke there was a sort of smile, which Elizabeth fancied she understood; he must be supposing her to be thinking of Jane and Netherfield, and she blushed as she answered,

"I do not mean to say that a woman may not be settled too near her family. The far and the near must be relative, and depend on many varying circumstances. Where there is fortune to make the expense of travelling unimportant, distance becomes no evil. But that is not the case here. Mr. and Mrs. Collins have a comfortable income, but not such a one as will allow of frequent journeys - and I am persuaded my friend would not call herself near her family under less than half the present distance."

Mr. Darcy drew his chair a little towards her, and said, "You cannot have a right to suchvery strong local attachment. You cannot have been always at

\section{Longbourn."}

Elizabeth looked surprised. The gentleman experienced some change of feeling; he drew back his chair, took a newspaper from the table, and, glancing over it, said, in a colder voice, "Are you pleased with Kent?"

A short dialogue on the subject of the country ensued, on either side calm and concise - and soon put an end to by the entrance of Charlotte and her sister, just returned from their walk. The tête-à-tête surprised them. Mr. Darcy related the mistake which had 
occasioned his intruding on Miss Bennet, and after sitting a few minutes longer without saying much to any body, went away.

"What can be the meaning of this!" said Charlotte, as soon as he was gone. "My dear Eliza he must be in love with you, or he would never have called on us in this familiar way."

But when Elizabeth told of his silence, it did not seem very likely, even to Charlotte's wishes, to be the case; and after various conjectures, they could at last only suppose his visit to proceed from the difficulty of finding anything to do, which was the more probable from the time of year. All field sports were over. Within doors there was Lady Catherine, books, and a billiard table, but gentlemen cannot be always within doors; and in the nearness of the Parsonage, or the pleasantness of the walk to it, or of the people who lived in it, the two cousins found a temptation from this period of walking thither almost every day. They called at various times of the morning, sometimes separately, sometimes together, and now and then accompanied by their aunt. It was plain to them all that Colonel Fitzwilliam came because he had pleasure in their society, a persuasion which of course recommended him still more; and Elizabeth was reminded by her own satisfaction in being with him, as well as by his evident admiration of her, of her former favourite George Wickham; and though, in comparing them, she saw there was less captivating softness in Colonel Fitzwilliam's manners, she believed he might have the best informed mind.

But why Mr. Darcy came so often to the Parsonage, it was more difficult to understand. It could not be for society, as he frequently sat there ten minutes together without opening his lips; and when he did speak, it seemed the effect of necessity rather 
than of choice - a sacrifice to propriety, not a pleasure to himself. He seldom appeared really animated. Mrs. Collins knew not what to make of him. Colonel Fitzwilliam's occasionally laughing at his stupidity, proved that he was generally different, which her own knowledge of him could not have told her; and as she would have liked to believe this change the effect of love, and the object of that love, her friend Eliza, she sat herself seriously to work to find it out. She watched him whenever they were at Rosings, and whenever he came to Hunsford; but without much success. He certainly looked at her friend a great deal, but the expression of that look was disputable. It was an earnest, steadfast gaze, but she often doubted whether there were much admiration in it, and sometimes it seemed nothing but absence of mind.

She had once or twice suggested to Elizabeth the possibility of his being partial to her, but Elizabeth always laughed at the idea; and Mrs. Collins did not think it right to press the subject, from the danger of raising expectations which might only end in disappointment; for in her opinion it admitted not of a doubt, that all her friend's dislike would vanish, if she could suppose him to be in her power.

In her kind schemes for Elizabeth, she sometimes planned her marrying Colonel Fitzwilliam. He was beyond comparison the pleasantest man; he certainly admired her, and his situation in life was most eligible; but, to counterbalance these advantages, $\mathrm{Mr}$. Darcy had considerable patronage in the church, and his cousin could have none at all.

30. According to Elizabeth, Mr. Collins is lucky to have Charlotte as a wife because Charlotte

A. and Mr. Collins are very much alike 
B. and Mr. Collins have a lot in common

C. gets along very well with Lady Catherine

D. had offers of marriage from other men

E. is an intelligent woman and still agreed to marry Mr. Collins.

31. The difference in social class between Darcy and Elizabeth becomes obvious when they discuss
A. Mr. Collins' marriage to Charlotte Lucas
B. the distance between Hunsford and Longbourn/Meryton
C. Mr. Bingley's wellbeing
D. Lady Catherine's willingness to renovate the parsonage
E. the countryside in Kent

32.Darcy and Elizabeth's conversation about the ease of travel best illustrates
A. Elizabeth's outspokenness
B. Darcy's insensitivity
C. Elizabeth and Darcy's propensity to disagree
D. Elizabeth's loyalty to her friend
E. Elizabeth and Darcy's inability to make small talk

33. Which of the following best describes the internal conflict portrayed in this passage?
A. Darcy's love of Elizabeth and his inability to admit it
B. Elizabeth's uncertainty about the reasons for Darcy's visits
C. Mrs. Collins's envy of both Elizabeth and Charlotte
D. Darcy's and Elizabeth's contrasting views on the ease of travel 
E. Charlotte's love for her husband and her desire to be home

34. In the third paragraph, the narrator remarks that Elizabeth and Darcy "then sat down, and when her enquiries after Rosings were made, seemed in danger of sinking into total silence. It was absolutely necessary, therefore, to think of something, and in this emergency recollecting when she had seen him last in Hertfordshire, and feeling curious to know what he would say on the subject of their hasty departure". Words such as "danger", "sinking" and "emergency" create a tone that can be described as which of the following?
A. awkward and tense
B. fearful and dangerous
C. honest and straightforward
D. angry and vindictive

35. Mr. Darcy drew his chair a little towards her, and said, "You cannot have a right to such very strong local attachment. You cannot have been always at Longbourn." Elizabeth is surprised at Darcy's remark because:

A. He may be implying that if she marries him, she would be living far from Longbourn B. It was socially inappropriate for Darcy to accuse Elizabeth for expressing her attachment to her family.

C. Elizabeth didn't know that Darcy disliked the neighborhood of Hertfordshire so much.

D. Darcy may be implying that Elizabeth wishes to move out of Longbourn before she is married. 


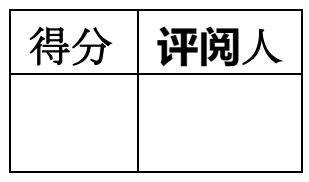

IV. Literature Discussion (20 points, 10 for each)

1. Read the quoted parts carefully and then answer the following question. Please write your answers on the Answer Sheet.

To be, or not to be: that is the question:

Whether 'tis nobler in the mind to suffer

The slings and arrows of outrageous fortune,

Or to take arms against a sea of troubles

And by opposing end them? To die: to sleep;

No more; and by a sleep to say we end

The heartache and the thousand natural shocks

That flesh is heir to, 'tis a consummation

Devoutly to be wish'd. ...

\section{Question:}

1.What is the internal conflict that Hamlet is going through?

2.The following poem "A Red, Red Rose" was written by Robert Burns. Read it carefully and then comment how Robert Burns developed the theme of the poem.

O, my Luve's ${ }^{[1]}$ like a red, red rose

That's newly sprung in June;

O, my Luve's like the melodie ${ }^{[2]}$,

That's sweetly play'd in tune. 
As fair art thou, my bonnie lass ${ }^{[3]}$, So deep in luve am I;

And I will luve thee still, my dear,

Till a' the seas gang dry, ${ }^{[4]}$

Till a' the seas gang dry, my dear,

And the rocks melt wi' the sun;

I will luve thee still, my dear,

While the sands o, ${ }^{[5]}$ life shall run.

And fare thee weel ${ }^{[6]}$, my only luve!

And fare thee weel a while!

And I will come again, my Luve,

Tho'it were ten thousand mile!

\section{Notes:}

[1]luve(Scotch): love.

[2]melodie: melody.

[3]lass (Scotch): a young woman; also sweetheart.

[4]Till a' the seas gang dry : Till all the seas go dry.

[5]o': of.

[6]fare thee weel: farewell, good-bye to you. 


\begin{tabular}{|l|l|}
\hline 得分 & 评阅人 \\
\hline & \\
& \\
\hline
\end{tabular}

V. Analyzing \&Interpreting Literature (20 points)

Satan is the best developed character in Paradise Lost. Examine the following lines, and write a comment that addresses the following questions:

1) How can you analyze the image of Satan based on your understanding of Paradise Lost?

2) What character traits that make Satan seem appealing or forgivable in this selected script?

Please justify your answer based on the social background, politics, religion, etc. at that time.

What though the field be lost?

All is not lost; the unconquerable will, And study of revenge, immortal hate, And courage never to submit or yield, And what is else not to be overcome; That glory never shall his wrath or might Extort from me. To bow or sue for grace With suppliant knee, and deify his power, Who from the terror of this arm so late Doubted his empire, that were low indeed, That were an ignominy and shame beneath 
This downfall; since, by fate, the strength of gods, And this empyreal substance, cannot fail, Since, through experience of this great event,

In arms not worse, in foresight much advanced,

We may with more successful hope resolve

To wage by force of guile eternal war

Irreconcilable to our grand foe,

Who now triumphs, and, in the excess of joy

Sole reigning, holds the tyranny of heaven. 


\title{
安徽农业大学 2015-2016 学年第一学期《英国文学史及 选读》试卷
}

\section{A 卷 参考答案及评分标准}

\section{Answer Key for CompTest3}

\author{
I: Cultural and Historical Context. (15points, 1.5for each)
}

1-5) ADACC 6-10) DBCBB

II. Identification of Themes, Purpose, and Plot Development (15 points, 1.5

for each)

11-15) BBCBC 16-20) AABCA

III: Reading Comprehension (30 points, 2 points for each)
a) Poetry Comprehension
21-24) ACAB
b) Literary Criticism Comprehension. 25-29) ECEBA
c) Literary Work Comprehension 30-35) EBAAAA

IV. Literature Discussion (20 points, 10 points for each)

1. "To be, or not to be" means "to live or not to live". Here Hamlet has meditation on death and life, and on how to bring back justice. Virtually, his melancholy and delay are well felt in the whole play. Here he wonders whether he should continue enduring the sufferings and wait for good chance for revenge or take action to kill his Uncle at once even at cost of his own life. Hamlet's melancholy and delay have been one of the heated topics in the study of Hamlet. As a humanist he has a lot advanced ideas and concepts as well as dreams and ambitions, as an intellectual he has 
much new knowledge and learning but he is lack of courage and bravery to take a risk.

2. Robert Burns wrote "A Red, Red Rose" as a traditional ballad, four verses of four lines each rhyming "abab". It begins with a quatrain containing two similes. Burns compares his love with a springtime blooming rose and then with a sweet melody. These are popular poetic images and this is the stanza most commonly quoted from the poem. The second and third stanzas become increasingly complex, ending with the metaphor of the "sands of life," or hourglass. On the one hand we are given the image of his love lasting until the seas run dry and the rocks melt with the sun, wonderfully poetic images. On the other hand Burns reminds us of the passage of time and the changes that result. That recalls the first stanza and its image of a red rose, newly sprung in June, which will change and decay with time. These are complex and competing images, typical of the more mature Robert Burns. The final stanza wraps up the poem's complexity with a farewell and a promise of return.

\section{Analyzing \& Interpreting Literature (20 points)}

Key words: very brave, smart and an image of a military hero; a driver of free will for freedom

References: some readers consider Satan to be the hero, or protagonist, of the story, because he struggles to overcome his own doubts and weaknesses and accomplishes his goal of corrupting humankind. This goal, however, is evil, and Adam and Eve are the moral heroes at the end of the story, as they help to begin humankind's slow process of 
redemption and salvation. Satan is far from being the story's object of admiration, as most heroes are. Nor does it make sense for readers to celebrate or emulate him, as they might with a true hero. Yet there are many compelling qualities to his character that make him intriguing to readers.

One source of Satan's fascination for us is that he is an extremely complex and subtle character. It would be difficult, perhaps impossible, for Milton to make perfect, infallible characters such as God the Father, God the Son, and the angels as interesting to read about as the flawed characters, such as Satan, Adam, and Eve. Satan, moreover, strikes a grand and majestic figure, apparently unafraid of being damned eternally, and uncowed by such terrifying figures as Chaos or Death. Many readers have argued that Milton deliberately makes Satan seem heroic and appealing early in the poem to draw us into sympathizing with him against our will, so that we may see how seductive evil is and learn to be more vigilant in resisting its appeal.

Milton devotes much of the poem's early books to developing Satan's character. Satan's greatest fault is his pride. He casts himself as an innocent victim, overlooked for an important promotion. But his ability to think so selfishly in Heaven, where all angels are equal and loved and happy, is surprising. His confidence in thinking that he could ever overthrow God displays tremendous vanity and pride. When Satan shares his pain and alienation as he reaches Earth in Book IV, we may feel somewhat sympathetic to him or even identify with him. But Satan continues to devote himself to evil. Every speech he gives is fraudulent and every story he tells is a lie. He works diligently to trick his fellow devils in Hell by having Beelzebub present Satan's own plan of action. 
Satan's character — or our perception of his character — changes significantly from Book I to his final appearance in Book X. In Book I he is a strong, imposing figure with great abilities as a leader and public statesmen, whereas by the poem's end he slinks back to Hell in serpent form. Satan's gradual degradation is dramatized by the sequence of different shapes he assumes. He begins the poem as a just-fallen angel of enormous stature, looks like a comet or meteor as he leaves Hell, then disguises himself as a more humble cherub, then as a cormorant, a toad, and finally a snake. His ability to reason and argue also deteriorates. In Book I, he persuades the devils to agree to his plan. In Book IV, however, he reasons to himself that the Hell he feels inside of him is reason to do more evil. When he returns to Earth again, he believes that Earth is more beautiful than Heaven, and that he may be able to live on Earth after all. Satan, removed from Heaven long enough to forget its unparalleled grandeur, is completely demented, coming to believe in his own lies. He is a picture of incessant intellectual activity without the ability to think morally. Once a powerful angel, he has become blinded to God's grace, forever unable to reconcile his past with his eternal punishment.

2) Satan is generally regarded as the best developed character in this masterpiece. He possesses "the unconquerable will", "study of revenge", "immortal hate", and "courage never to submit or yield". As a "fallen hero", Satan dares to challenge the unjust authority-God and to pursue equality. In a word, Milton successfully embodied the revolutionary spirit in Satan.

From the perspective of Satan and his followers, rebellion against God was inevitable. Heaven demanded obedience and servitude. The revolt may have failed, but it 
has left them their freedom. In this way the poem begins to contrast an analogy with the rebellion to the Civil War and with Milton's own interrogation of established authority. Another reason that Satan is easy to be sympathized with is that he is much like us than God or the Son is. As the embodiment of human errors, he is much easier for us to imagine and empathize with than an omniscient deity. Satan's character and psychology are all very human, and his envy, pride and despair are understandable given his situation.

Furthermore, Satan is good at making persuasive and moving speech and his arguments are often compelling: he claims the angels have liberty and he opposes the hierarchies of heaven. In many ways Satan becomes more understandable in this speech for his human qualities, and he becomes more interesting as well due to the unpredictability of his character. 


\section{Appendix $\mathrm{O}$}

\section{Statistical Models}

\section{ANOVA}

According to Hinkle, Wiersma and Jurs (2003), ANOVA is the procedure for testing the hypothesis that more than two population means are all equal. The respective alternative hypothesis is that not all population means are equal. A statistical significant ANOVA is usually followed by a pairwise comparison to identify which two population means differ significantly.

An ANOVA with one independent variable is also called one-way ANOVA. The main idea of ANOVA is the decomposition of variation on the dependent variable. In one-way ANOVA, the total variance is partitioned into two sources, namely the within group variation and between group variation, where the latter reflects not only the variation caused by random sampling but also due to differential treatment effects. The null hypothesis is tested by computing the ratio of the between group variation estimate and the within group variance estimate. The sampling distribution of the test statistic is the F distribution. The computed $\mathrm{F}$ value is compared with the $\mathrm{F}$ critical value, which is determined by the significance level $\alpha$ as well as the degree of freedom for the estimates of both the between group variation and the within group variance.

\section{ANCOVA and Simple Group Main Effects Tests}

ANCOVA allows statistical control for more precise quantification of the variation in the dependent variable that are attributed by independent variables. It partitions out the variation that is attributed to an extraneous variable, i.e. the covariate, 
which results in a smaller error variance. In particular, ANCOVA partitions the total variation in the dependent variable into three components: the within group variation, the between group variation and the variation due to covariates.

The null hypothesis and alternative hypothesis of ANCOVA is very similar to the counterpart statements in ANOVA. The only difference is that the population means are the adjusted ones from the computing of variation for covariates.

There are two assumptions for ANCOVA which are additional to the Simple Group Main Effects Test. The first one is that ANCOVA assumes a linear relationship between the dependent variable and the covariate. Non-linear relationship will result in a biased ANCOVA result. The second additional assumption is called homogeneity of regression/slopes. It requires the same slope of regression lines within each of the groups. A test of the homogeneity-of-slopes is prerequisite to ANCOVA. Failure of this test usually results a follow-up simple group main effects test for particular covariate values at low, medium and high level.

\section{MANOVA}

MANOVA is an extension from ANOVA. It includes multiple dependent variables instead of just one. As a consequence, the null hypothesis for MANOVA is that the population means are the same for each of dependent variables. A rejection to the null hypothesis indicates that not all population means are the same on every dependent variable. A statistical significant MANOVA is usually followed by a multiple comparison in order to identify which two population means for which dependent variable are statistically different. 
MANOVA assumes one additional fact from ANOVA. The population covariance among the dependent variables are the same.

\section{MANCOVA}

Comparing to ANCOVA, MANCOVA is an extension which includes multiple dependent variables at a time. Comparing to MANOVA, MANCOVA provides better statistical control by introducing the extraneous variable as covariate and allows further decomposition of the total variance. All the assumptions for ANOVA, ANCOVA, MANOVA applied to the MANCOVA.

\section{Repeated Measure Designs and Mixed Designs}

In general, repeated measure designs take multiple measurements of subjects for two or more times on dependent variables. The measurements can be taken under different conditions or at different times. For simple repeated measure ANOVA, the total variation is partitioned into the between-subject variation, the within-subject variation and the remaining variation which is also called residual variation. Here the test occasion or time is the effect of primary interest. In other words, the null hypothesis can be represented as there is no difference among population means for different test occasions or times. The hypothesis is tested by comparing to the critical value the F statistics, which equals the ratio of within-subject variation estimate (i.e. the mean square) and the residual variance estimate. Simple repeated measure ANOVA takes two additional assumptions: (a) the population variances for test occasions or times are equal, and (b) the population correlation coefficients between pairs of test occasion or time scores are equal. 
An extension to simple repeated measure ANOVA is the one-way repeated measure ANOVA. The latter investigates not only the effect of test occasion/time (within-subject variation) but also the effect of the treatment (between-group variation). This is also called a mixed design. A further extension is the one-way repeated measure ANCOVA, where the partitioning of total variance is adjusted with the variance introduced by the covariate. A final extension is the one way repeated measure MANCOVA, where the measured dependent variables are in multi-dimensional space. The mixed designs are used within this dissertation in Chapter CHAPTER IV. 
Appendix P

\section{Supplementary Tables}

Table 26

Descriptive Statistics for CulTestA

\begin{tabular}{lllll}
\hline Group N & Mean & $\begin{array}{l}\text { Std. } \\
\text { Deviation }\end{array}$ & Std. Error \\
\hline 1 & 26 & 54.08 & 15.97 & 3.13 \\
2 & 28 & 57.67 & 19.46 & 3.67 \\
3 & 52 & 40.25 & 13.89 & 1.92 \\
Total & 106 & 48.24 & 17.76 & 1.72 \\
\hline
\end{tabular}

Table 27

Descriptive Statistics for CulTestB

\begin{tabular}{lllc}
\hline Group & Mean & \multicolumn{2}{l}{ Std. Deviation N } \\
\hline 1 & 68.31 & 14.04 & 26 \\
2 & 78.27 & 11.81 & 28 \\
3 & 40.07 & 14.18 & 52 \\
Total & 57.08 & 21.79 & 106 \\
\hline
\end{tabular}

Table 28

Descriptive Statistics for CulTest Improvement scores

\begin{tabular}{llll}
\hline Group & Mean & \multicolumn{2}{l}{ Std. Deviation N } \\
\hline 1 & 14.23 & 13.42 & 26 \\
2 & 20.61 & 13.43 & 28 \\
3 & -.18 & 19.90 & 52
\end{tabular}


$\begin{array}{llll}\text { Total } & 8.84 & 19.13 & 106\end{array}$

Table 29

Descriptive Statistics for Three CompTests

\begin{tabular}{lllll}
\hline & group & Mean & \multicolumn{2}{l}{ Std. Deviation N } \\
\hline CompTest1 & 1 & 71.02 & 8.41 & 26 \\
& 2 & 71.34 & 10.31 & 28 \\
& 3 & 61.84 & 11.90 & 52 \\
& Total & 66.60 & 11.62 & 106 \\
CompTest2 & 1 & 64.54 & 9.63 & 26 \\
& 2 & 66.38 & 10.23 & 28 \\
& 3 & 56.78 & 11.54 & 52 \\
& Total & 61.21 & 11.54 & 106 \\
CompTest3 & 1 & 71.96 & 7.53 & 26 \\
& 2 & 74.79 & 8.55 & 28 \\
& 3 & 69.84 & 10.55 & 52 \\
& Total & 71.67 & 9.52 & 106 \\
\hline
\end{tabular}

Table 30

Descriptive Statistics for TEM4

\begin{tabular}{lllll}
\hline & $\mathrm{N}$ & Mean & $\begin{array}{l}\text { Std. } \\
\text { Deviation }\end{array}$ & Std. Error \\
\hline 1 & 26 & 65.08 & 5.48 & 1.08 \\
2 & 28 & 65.71 & 6.59 & 1.24 \\
3 & 52 & 66.63 & 6.98 & .97 \\
Total & 106 & 66.01 & 6.51 & .63 \\
\hline
\end{tabular}


Table 31

Estimated 95\% Confidence Intervals of Mean in CulTestB Conditioned at Low CulTestA

\begin{tabular}{lllll}
\hline Group & Mean & Std. Error & \multicolumn{2}{l}{$95 \%$ Confidence Interval } \\
\cline { 3 - 5 } & & & Lower Bound & Upper Bound \\
\hline 1 & $55.72^{\mathrm{a}}$ & 4.36 & 47.07 & 64.37 \\
2 & $66.15^{\mathrm{a}}$ & 4.03 & 58.13 & 74.16 \\
3 & $40.10^{\mathrm{a}}$ & 2.09 & 35.96 & 44.26 \\
\hline
\end{tabular}

Note. ${ }^{\text {a }}$ Covariates appearing in the model are evaluated at the following values: CulTestA $=30.48$.

Table 32

Estimated 95\% Confidence Intervals of Mean in CulTestB Conditioned at Medium CulTestA

\begin{tabular}{lllll}
\hline Group & Mean & Std. Error & \multicolumn{2}{l}{$95 \%$ Confidence Interval } \\
\cline { 3 - 5 } & & & Lower Bound & Upper Bound \\
\hline 1 & $65.19^{\mathrm{a}}$ & 2.57 & 60.09 & 70.30 \\
2 & $74.07^{\mathrm{a}}$ & 2.59 & 68.93 & 79.20 \\
3 & $40.03^{\mathrm{a}}$ & 1.97 & 36.12 & 43.94
\end{tabular}

Note. ${ }^{\text {a }}$ Covariates appearing in the model are evaluated at the following values: CulTest $\mathrm{A}=48.24$.

Table 33

Estimated 95\% Confidence Intervals of Mean in CulTestB Conditioned at High CulTestA

\begin{tabular}{lllll}
\hline Group & Mean & Std. Error & \multicolumn{2}{l}{$95 \%$ Confidence Interval } \\
\cline { 3 - 5 } & & & Lower Bound & Upper Bound \\
\hline 1 & $74.67^{\mathrm{a}}$ & 3.02 & 68.66 & 80.68 \\
2 & $81.99^{\mathrm{a}}$ & 2.53 & 76.96 & 87.01 \\
3 & $39.96^{\mathrm{a}}$ & 3.61 & 32.78 & 47.13 \\
\hline
\end{tabular}

Note. ${ }^{a}$ Covariates appearing in the model are evaluated at the following values: CulTestA $=66.00$. 
Table 34

Descriptive Statistics for Five Cultural Components in CulTestB

\begin{tabular}{|c|c|c|c|c|}
\hline & Group & Mean & Std. & \\
\hline \multirow{4}{*}{ TestB_Custom } & 1 & 14.17 & 3.18 & 26 \\
\hline & 2 & 15.93 & 2.30 & 28 \\
\hline & 3 & 9.42 & 2.88 & 52 \\
\hline & Total & 12.31 & 4.03 & 106 \\
\hline \multirow[t]{4}{*}{ TestB_Econ } & 1 & 14.17 & 3.20 & 26 \\
\hline & 2 & 15.98 & 2.80 & 28 \\
\hline & 3 & 9.44 & 4.20 & 52 \\
\hline & Total & 12.33 & 4.64 & 106 \\
\hline \multirow[t]{4}{*}{ TestB_Gndr } & 1 & 13.96 & 3.67 & 26 \\
\hline & 2 & 15.09 & 3.09 & 28 \\
\hline & 3 & 8.94 & 4.05 & 52 \\
\hline & Total & 11.80 & 4.66 & 106 \\
\hline \multirow[t]{4}{*}{ TestB_Pol } & 1 & 12.15 & 3.18 & 26 \\
\hline & 2 & 15.32 & 2.96 & 28 \\
\hline & 3 & 4.84 & 4.53 & 52 \\
\hline & Total & 9.40 & 6.02 & 106 \\
\hline \multirow[t]{4}{*}{ TestB_Free } & 1 & 13.85 & 3.35 & 26 \\
\hline & 2 & 15.95 & 2.55 & 28 \\
\hline & 3 & 7.42 & 4.62 & 52 \\
\hline & Total & 11.25 & 5.43 & 106 \\
\hline
\end{tabular}

Table 35

Estimated 95\% Confidence Intervals of Mean in Five Cultural Components for CulTestB

Dependent Variable $\quad$ Group $\quad$ Mean $\quad$ Std. Error $95 \%$ Confidence Interval




\begin{tabular}{|c|c|c|c|c|c|}
\hline & & & & Lower Bound & Upper Bound \\
\hline \multirow[t]{3}{*}{ TestB_Custom } & 1 & $13.87^{\mathrm{a}}$ & .54 & 12.80 & 14.94 \\
\hline & 2 & $15.44^{\mathrm{a}}$ & .54 & 14.38 & 16.50 \\
\hline & 3 & $9.84^{\mathrm{a}}$ & .40 & 9.05 & 10.63 \\
\hline \multirow[t]{3}{*}{ TestB_Econ } & 1 & $13.75^{\mathrm{a}}$ & .69 & 12.38 & 15.12 \\
\hline & 2 & $15.30^{\mathrm{a}}$ & .69 & 13.94 & 16.66 \\
\hline & 3 & $10.02^{\mathrm{a}}$ & .51 & 9.01 & 11.04 \\
\hline \multirow[t]{3}{*}{ TestB_Gndr } & 1 & $13.52^{\mathrm{a}}$ & .71 & 12.12 & 14.921 \\
\hline & 2 & $14.37^{\mathrm{a}}$ & .70 & 12.99 & 15.76 \\
\hline & 3 & $9.55^{\mathrm{a}}$ & .52 & 8.52 & 10.58 \\
\hline \multirow[t]{3}{*}{ TestB_Pol } & 1 & $11.87^{\mathrm{a}}$ & .76 & 10.37 & 13.38 \\
\hline & 2 & $14.87^{\mathrm{a}}$ & .75 & 13.37 & 16.36 \\
\hline & 3 & $5.22^{\mathrm{a}}$ & .56 & 4.11 & 6.33 \\
\hline \multirow[t]{3}{*}{ TestB_Free } & 1 & $13.55^{\mathrm{a}}$ & .76 & 12.04 & 15.06 \\
\hline & 2 & $15.48^{\mathrm{a}}$ & .75 & 13.98 & 16.97 \\
\hline & 3 & $7.82^{\mathrm{a}}$ & .56 & 6.71 & 8.94 \\
\hline
\end{tabular}

Note. ${ }^{a}$ Covariates appearing in the model are evaluated at the following values: CulTestA $=48.2406$.

Table 36

95\% Confidence Intervals of Mean for General Improvement Scores in CulTests

\begin{tabular}{lllll}
\hline Group & Mean & Std. Error & \multicolumn{2}{l}{$95 \%$ Confidence Interval } \\
\cline { 3 - 5 } & & & Lower Bound & Upper Bound \\
\hline 1 & 14.23 & 3.32 & 7.64 & 20.82 \\
2 & 20.61 & 3.20 & 14.26 & 26.96 \\
3 & -.18 & 2.35 & -4.84 & 4.48 \\
\hline
\end{tabular}


Table 37

Descriptive Statistics for Improvement Scores in Five Cultural Components of CulTestB

\begin{tabular}{|c|c|c|c|c|}
\hline & Group & Mean & Std. Deviation & $\mathrm{N}$ \\
\hline \multirow[t]{4}{*}{ ImproveCustom } & 1 & 1.56 & 4.58 & 26 \\
\hline & 2 & 3.29 & 2.96 & 28 \\
\hline & 3 & -.96 & 4.62 & 52 \\
\hline & Total & .78 & 4.58 & 106 \\
\hline \multirow[t]{4}{*}{ ImprvoeEco } & 1 & 4.44 & 4.91 & 26 \\
\hline & 2 & 5.07 & 3.57 & 28 \\
\hline & 3 & 2.89 & 5.70 & 52 \\
\hline & Total & 3.85 & 5.08 & 106 \\
\hline \multirow[t]{4}{*}{ ImproveGndr } & 1 & 4.38 & 4.91 & 26 \\
\hline & 2 & 3.70 & 3.84 & 28 \\
\hline & 3 & 4.57 & 6.34 & 52 \\
\hline & Total & 4.29 & 5.40 & 106 \\
\hline \multirow[t]{4}{*}{ ImprovePol } & 1 & 1.98 & 4.42 & 26 \\
\hline & 2 & 4.55 & 3.00 & 28 \\
\hline & 3 & -4.64 & 6.58 & 52 \\
\hline & Total & -.59 & 6.69 & 106 \\
\hline \multirow[t]{4}{*}{ ImproveFree } & 1 & -1.83 & 3.07 & 26 \\
\hline & 2 & -4.04 & 4.22 & 28 \\
\hline & 3 & 2.04 & 6.69 & 52 \\
\hline & Total & -.51 & 5.96 & 106 \\
\hline
\end{tabular}


Table 38

Estimated 95\% Confidence Intervals of Mean for Improvement Scores in Five Cultural Components of CulTests

\begin{tabular}{|c|c|c|c|c|c|}
\hline \multirow[t]{2}{*}{$\overline{\text { Dependent Variable }}$} & \multirow[t]{2}{*}{ Group } & \multirow[t]{2}{*}{ Mean } & \multirow[t]{2}{*}{ Std. Error } & \multicolumn{2}{|c|}{$95 \%$ Confidence Interval } \\
\hline & & & & $\overline{\text { Lower Bound }}$ & Upper Bound \\
\hline \multirow[t]{3}{*}{ ImproveCustom } & 1 & 1.56 & .83 & -.09 & 3.21 \\
\hline & 2 & 3.29 & .80 & 1.70 & 4.88 \\
\hline & 3 & -.96 & .59 & -2.12 & .20 \\
\hline \multirow[t]{3}{*}{ ImprvoeEco } & 1 & 4.44 & .99 & 2.49 & 6.40 \\
\hline & 2 & 5.07 & .95 & 3.18 & 6.96 \\
\hline & 3 & 2.89 & .70 & 1.51 & 4.28 \\
\hline \multirow[t]{3}{*}{ ImproveGndr } & 1 & 4.39 & 1.07 & 2.27 & 6.50 \\
\hline & 2 & 3.70 & 1.02 & 1.66 & 5.74 \\
\hline & 3 & 4.57 & .76 & 3.07 & 6.06 \\
\hline \multirow[t]{3}{*}{ ImprovePol } & 1 & 1.98 & 1.05 & -.10 & 4.06 \\
\hline & 2 & 4.55 & 1.01 & 2.55 & 6.56 \\
\hline & 3 & -4.64 & .74 & -6.11 & -3.18 \\
\hline \multirow[t]{3}{*}{ ImproveFree } & 1 & -1.83 & 1.06 & -3.93 & .27 \\
\hline & 2 & -4.04 & 1.02 & -6.06 & -2.01 \\
\hline & 3 & 2.04 & .75 & .56 & 3.52 \\
\hline
\end{tabular}

Table 39

Estimated 95\% Confidence Intervals of Mean for Group in Three CompTests

\begin{tabular}{lllll}
\hline Group & Mean & Std. Error & \multicolumn{2}{l}{$95 \%$ Confidence Interval } \\
\cline { 3 - 4 } & & & Lower Bound & Upper Bound \\
\hline 1 & $68.76^{\mathrm{a}}$ & 1.43 & 65.93 & 71.60
\end{tabular}




\begin{tabular}{lllll}
2 & $70.12^{\mathrm{a}}$ & 1.37 & 67.40 & 72.85 \\
3 & $61.82^{\mathrm{a}}$ & 1.01 & 59.81 & 63.82 \\
\hline
\end{tabular}

Note. ${ }^{\text {a }}$ Covariates appearing in the model are evaluated at the following values: TEM4 $=66.0094$.

Table 40

Estimated 95\% Confidence Intervals of Mean for Each Group in Different CompTest

\begin{tabular}{llllll}
\hline Group & Time & Mean & Std. Error & \multicolumn{2}{l}{$95 \%$ Confidence Interval } \\
\cline { 5 - 6 } & & & & Lower Bound & Upper Bound \\
\hline 1 & 1 & $71.68^{\mathrm{a}}$ & 1.91 & 67.89 & 75.47 \\
& 2 & $65.00^{\mathrm{a}}$ & 2.03 & 60.98 & 69.02 \\
& 3 & $69.60^{\mathrm{a}}$ & 1.59 & 66.44 & 72.76 \\
& 1 & $71.55^{\mathrm{a}}$ & 1.84 & 67.90 & 75.19 \\
& 2 & $66.52^{\mathrm{a}}$ & 1.95 & 62.66 & 70.39 \\
& 3 & $72.30^{\mathrm{a}}$ & 1.53 & 69.26 & 75.33 \\
& 1 & $61.39^{\mathrm{a}}$ & 1.35 & 58.71 & 64.08 \\
& 2 & $56.47^{\mathrm{a}}$ & 1.43 & 53.63 & 59.31 \\
& 3 & $67.59^{\mathrm{a}}$ & 1.17 & 65.36 & 69.82
\end{tabular}

Note. ${ }^{\mathrm{a}}$ Covariates appearing in the model are evaluated at the following values: TEM4 $=66.0094$.

Table 41

Estimated 95\% Confidence Intervals of Mean for Each CompTest in Different Group

\begin{tabular}{llllll}
\hline Time & Group & Mean & Std. Error & \multicolumn{2}{l}{$95 \%$ Confidence Interval } \\
\cline { 5 - 6 } & & & & Lower Bound & Upper Bound \\
\hline 1 & 1 & $71.67^{\mathrm{a}}$ & 1.91 & 67.89 & 75.47 \\
& 2 & $71.59^{\mathrm{a}}$ & 1.84 & 67.90 & 75.20 \\
& 3 & $61.39^{\mathrm{a}}$ & 1.35 & 58.71 & 64.08 \\
& 1 & $65.00^{\mathrm{a}}$ & 2.03 & 60.98 & 69.02 \\
& 2 & $66.52^{\mathrm{a}}$ & 1.95 & 62.67 & 70.39
\end{tabular}




\begin{tabular}{llllll} 
& 3 & $56.47^{\mathrm{a}}$ & 1.43 & 53.63 & 59.31 \\
3 & 1 & $69.60^{\mathrm{a}}$ & 1.59 & 66.44 & 72.76 \\
& 2 & $72.30^{\mathrm{a}}$ & 1.53 & 69.26 & 75.33 \\
& 3 & $67.59^{\mathrm{a}}$ & 1.13 & 65.36 & 69.82 \\
\hline
\end{tabular}

Note ${ }^{\text {a }}$ Covariates appearing in the model are evaluated at the following values: TEM4 $=66.0094$.

Table 42

Descriptive Statistics for Three Subcategories in CompTests

\begin{tabular}{|c|c|c|c|c|}
\hline & Group & Mean & Std. Deviation & $\mathrm{N}$ \\
\hline \multirow{4}{*}{$\overline{\mathrm{CC} 1 \mathrm{~N}}$} & 1 & 76.15 & 14.72 & 26 \\
\hline & 2 & 74.64 & 16.21 & 28 \\
\hline & 3 & 73.65 & 12.53 & 52 \\
\hline & Total & 74.53 & 14.02 & 106 \\
\hline \multirow[t]{4}{*}{$\mathrm{CC} 2 \mathrm{~N}$} & 1 & 59.62 & 16.37 & 26 \\
\hline & 2 & 71.79 & 13.89 & 28 \\
\hline & 3 & 61.92 & 15.09 & 52 \\
\hline & Total & 63.969 & 15.72 & 106 \\
\hline \multirow[t]{4}{*}{$\mathrm{CC} 3 \mathrm{~N}$} & 1 & 71.54 & 12.87 & 26 \\
\hline & 2 & 75.71 & 11.68 & 28 \\
\hline & 3 & 69.81 & 12.91 & 52 \\
\hline & Total & 71.79 & 12.71 & 106 \\
\hline \multirow[t]{4}{*}{ ID1N } & 1 & 55.00 & 11.04 & 26 \\
\hline & 2 & 61.79 & 14.16 & 28 \\
\hline & 3 & 60.96 & 14.32 & 52 \\
\hline & Total & 59.72 & 13.69 & 106 \\
\hline \multirow[t]{3}{*}{ ID2N } & 1 & 71.15 & 13.07 & 26 \\
\hline & 2 & 75.71 & 12.89 & 28 \\
\hline & 3 & 64.42 & 15.52 & 52 \\
\hline
\end{tabular}




\begin{tabular}{|c|c|c|c|c|}
\hline & Total & 69.06 & 14.96 & 106 \\
\hline \multirow[t]{4}{*}{ ID3N } & 1 & 51.54 & 15.15 & 26 \\
\hline & 2 & 55.71 & 18.14 & 28 \\
\hline & 3 & 56.92 & 15.15 & 52 \\
\hline & Total & 55.28 & 15.99 & 106 \\
\hline \multirow[t]{4}{*}{ LA1N } & 1 & 77.23 & 14.64 & 26 \\
\hline & 2 & 74.46 & 11.60 & 28 \\
\hline & 3 & 56.37 & 19.30 & 52 \\
\hline & Total & 66.26 & 19.05 & 106 \\
\hline \multirow[t]{4}{*}{ LA2N } & 1 & 63.69 & 12.26 & 26 \\
\hline & 2 & 58.93 & 14.22 & 28 \\
\hline & 3 & 50.50 & 17.64 & 52 \\
\hline & Total & 55.96 & 16.46 & 106 \\
\hline \multirow[t]{4}{*}{ LA3N } & 1 & 76.43 & 8.24 & 26 \\
\hline & 2 & 81.58 & 16.60 & 28 \\
\hline & 3 & 72.66 & 12.77 & 52 \\
\hline & Total & 75.94 & 13.42 & 106 \\
\hline
\end{tabular}

Table 43

Estimated 95\% Confidence Intervals of Mean for Group in Three Subcategories of CompTests

\begin{tabular}{llllll}
\hline Measure & Group & Mean & Std. Error & \multicolumn{2}{l}{$95 \%$ Confidence Interval } \\
\cline { 4 - 6 } & & & & Lower Bound & Upper Bound \\
\hline CC & 1 & $69.64^{\mathrm{a}}$ & 1.71 & 66.24 & 73.03 \\
& 2 & $74.22^{\mathrm{a}}$ & 1.65 & 70.95 & 77.48 \\
& 3 & $68.10^{\mathrm{a}}$ & 1.21 & 65.70 & 70.51 \\
$\mathrm{ID}$ & 1 & $59.82^{\mathrm{a}}$ & 1.92 & 56.01 & 63.63 \\
& 2 & $64.59^{\mathrm{a}}$ & 1.85 & 60.93 & 68.25
\end{tabular}




$\begin{array}{llllll} & 3 & 60.38^{\mathrm{a}} & 1.36 & 57.68 & 63.07 \\ \text { LA } & 1 & 72.98^{\mathrm{a}} & 1.91 & 69.19 & 76.78 \\ & 2 & 71.82^{\mathrm{a}} & 1.84 & 68.18 & 75.48 \\ & 3 & 59.49^{\mathrm{a}} & 1.35 & 56.80 & 62.17\end{array}$

Notes. ${ }^{a}$ Covariates appearing in the model are evaluated at the following values: TEM4 $=66.0094$.

Table 44

Estimated 95\% Confidence Intervals of Mean for Each Group in Three Subcategories of Different CompTest

\begin{tabular}{|c|c|c|c|c|c|c|}
\hline \multirow[t]{2}{*}{$\overline{\text { Measure }}$} & \multirow[t]{2}{*}{ Group } & \multirow[t]{2}{*}{ Time } & \multirow[t]{2}{*}{ Mean } & \multirow[t]{2}{*}{ Std. Error } & \multicolumn{2}{|c|}{ 95\% Confidence Interval } \\
\hline & & & & & $\overline{\text { Lower Bound }}$ & Upper Bound \\
\hline \multirow[t]{9}{*}{$\overline{\mathrm{CC}}$} & 1 & 1 & $76.91^{\mathrm{a}}$ & 2.58 & 71.78 & 82.04 \\
\hline & & 2 & $60.06^{\mathrm{a}}$ & 2.92 & 54.27 & 65.86 \\
\hline & & 3 & $71.93^{\mathrm{a}}$ & 2.43 & 67.12 & 76.75 \\
\hline & 2 & 1 & $74.88^{\mathrm{a}}$ & 2.48 & 69.95 & 79.81 \\
\hline & & 2 & $71.93^{\mathrm{a}}$ & 2.81 & 66.36 & 77.50 \\
\hline & & 3 & $75.84^{\mathrm{a}}$ & 2.33 & 71.21 & 80.47 \\
\hline & 3 & 1 & $73.15^{\mathrm{a}}$ & 1.83 & 69.52 & 76.77 \\
\hline & & 2 & $61.62^{\mathrm{a}}$ & 2.07 & 57.53 & 65.72 \\
\hline & & 3 & $69.54^{\mathrm{a}}$ & 1.72 & 66.14 & 72.94 \\
\hline \multirow[t]{7}{*}{ ID } & 1 & 1 & $55.36^{\mathrm{a}}$ & 2.63 & 50.15 & 60.58 \\
\hline & & 2 & $71.84^{\mathrm{a}}$ & 2.69 & 66.57 & 77.11 \\
\hline & & 3 & $52.25^{\mathrm{a}}$ & 3.00 & 46.29 & 58.20 \\
\hline & 2 & 1 & $61.90^{\mathrm{a}}$ & 2.53 & 56.89 & 66.92 \\
\hline & & 2 & $75.93^{\mathrm{a}}$ & 2.56 & 70.86 & 81.00 \\
\hline & & 3 & $55.94^{\mathrm{a}}$ & 2.89 & 50.21 & 61.66 \\
\hline & 3 & 1 & $60.72^{\mathrm{a}}$ & 1.86 & 57.03 & 64.40 \\
\hline
\end{tabular}




\begin{tabular}{|c|c|c|c|c|c|c|}
\hline & & 2 & $63.96^{\mathrm{a}}$ & 1.88 & 60.24 & 67.69 \\
\hline & & 3 & $56.45^{\mathrm{a}}$ & 2.12 & 52.24 & 60.66 \\
\hline \multirow[t]{9}{*}{ LA } & 1 & 1 & $77.97^{\mathrm{a}}$ & 3.09 & 71.84 & 84.10 \\
\hline & & 2 & $64.06^{\mathrm{a}}$ & 3.04 & 58.03 & 70.09 \\
\hline & & 3 & $76.92^{\mathrm{a}}$ & 2.48 & 71.99 & 81.84 \\
\hline & 2 & 1 & $74.70^{\mathrm{a}}$ & 2.97 & 68.81 & 80.59 \\
\hline & & 2 & $59.05^{\mathrm{a}}$ & 2.92 & 53.25 & 64.84 \\
\hline & & 3 & $81.74^{\mathrm{a}}$ & 2.39 & 77.00 & 86.47 \\
\hline & 3 & 1 & $55.87^{\mathrm{a}}$ & 2.19 & 51.54 & 60.20 \\
\hline & & 2 & $50.25^{\mathrm{a}}$ & 2.15 & 45.99 & 54.52 \\
\hline & & 3 & $72.34^{\mathrm{a}}$ & 1.75 & 68.86 & 75.82 \\
\hline
\end{tabular}

Note. ${ }^{\text {a }}$ Covariates appearing in the model are evaluated at the following values: TEM4 =66.0094.

Table 45

Estimated 95\% Confidence Intervals of Mean for Three Subcategories of Each CompTest in Different Group

\begin{tabular}{|c|c|c|c|c|c|c|}
\hline \multirow[t]{2}{*}{ Measure } & \multirow[t]{2}{*}{ Time } & \multirow[t]{2}{*}{ Group } & \multirow[t]{2}{*}{ Mean } & \multirow[t]{2}{*}{ Std. Error } & \multicolumn{2}{|c|}{$95 \%$ Confidence Interval } \\
\hline & & & & & $\overline{\text { Lower Bound }}$ & Upper Bound \\
\hline \multirow[t]{9}{*}{$\overline{\mathrm{CC}}$} & \multirow[t]{3}{*}{1} & 1 & $76.91^{\mathrm{a}}$ & 2.58 & 71.78 & 82.04 \\
\hline & & 2 & $74.88^{\mathrm{a}}$ & 2.48 & 69.95 & 79.81 \\
\hline & & 3 & $73.15^{\mathrm{a}}$ & 1.83 & 69.52 & 76.77 \\
\hline & \multirow[t]{3}{*}{2} & 1 & $60.06^{\mathrm{a}}$ & 2.92 & 54.27 & 65.86 \\
\hline & & 2 & $71.93^{\mathrm{a}}$ & 2.81 & 66.36 & 77.50 \\
\hline & & 3 & $61.62^{\mathrm{a}}$ & 2.07 & 57.53 & 65.72 \\
\hline & \multirow[t]{3}{*}{3} & 1 & $71.93^{\mathrm{a}}$ & 2.43 & 67.12 & 76.75 \\
\hline & & 2 & $75.84^{\mathrm{a}}$ & 2.33 & 71.21 & 80.47 \\
\hline & & 3 & $69.54^{\mathrm{a}}$ & 1.72 & 66.14 & 72.95 \\
\hline ID & 1 & 1 & $55.36^{\mathrm{a}}$ & 2.63 & 50.15 & 60.58 \\
\hline
\end{tabular}




\begin{tabular}{|c|c|c|c|c|c|c|}
\hline & 2 & $61.90^{\mathrm{a}}$ & 2.53 & 56.89 & 66.92 \\
\hline & & 3 & $60.72^{\mathrm{a}}$ & 1.86 & 57.03 & 64.40 \\
\hline & 2 & 1 & $71.84^{\mathrm{a}}$ & 2.66 & 66.57 & 77.11 \\
\hline & & 2 & $75.93^{\mathrm{a}}$ & 2.56 & 70.86 & 81.00 \\
\hline & & 3 & $63.96^{\mathrm{a}}$ & 1.880 & 60.24 & 67.69 \\
\hline & 3 & 1 & $52.25^{\mathrm{a}}$ & 3.00 & 46.29 & 58.20 \\
\hline & & 2 & $55.94^{\mathrm{a}}$ & 2.89 & 50.21 & 61.67 \\
\hline & & 3 & $56.45^{\mathrm{a}}$ & 2.12 & 52.24 & 60.66 \\
\hline \multirow[t]{9}{*}{ LA } & 1 & 1 & $77.97^{\mathrm{a}}$ & 3.09 & 71.84 & 84.10 \\
\hline & & 2 & $74.70^{\mathrm{a}}$ & 2.97 & 68.81 & 80.59 \\
\hline & & 3 & $55.87^{\mathrm{a}}$ & 2.19 & 51.54 & 60.20 \\
\hline & 2 & 1 & $64.06^{\mathrm{a}}$ & 3.04 & 58.03 & 70.09 \\
\hline & & 2 & $59.05^{\mathrm{a}}$ & 2.92 & 53.25 & 64.84 \\
\hline & & 3 & $50.25^{\mathrm{a}}$ & 2.15 & 45.99 & 54.52 \\
\hline & 3 & 1 & $76.92^{\mathrm{a}}$ & 2.48 & 71.99 & 81.84 \\
\hline & & 2 & $81.74^{\mathrm{a}}$ & 2.39 & 77.00 & 86.47 \\
\hline & & 3 & $72.34^{\mathrm{a}}$ & 1.75 & 68.86 & 75.82 \\
\hline
\end{tabular}

Note. a Covariates appearing in the model are evaluated at the following values: TEM4 =66.0094. 


\section{VITA}

\section{YU ZHANG}

2008

2011

2013

2014-2017

B.A. English Language and Literature Anhui Agricultural University Hefei, China

M.A. Theory of Literature and Arts Anhui University Hefei, China

Ed.S. Curriculum \& Instruction Florida International University Miami, Florida

Doctoral Candidate

Florida International University Miami, Florida 